\title{
Clinical Practice Guideline for Cardiac Rehabilitation in Korea
}

\author{
Chul Kim, MD, $\mathrm{PhD}^{1}$, Jidlong Sung, MD, PhD², Jong Hwa Lee, MD, PhD ${ }^{3}$, Won-Seok Kim, MD, $\mathrm{PhD}^{4,5}$, \\ Goo Joo Lee, $\mathrm{MD}^{6}$, Sungju Jee, $\mathrm{MD}, \mathrm{PhD}^{7}$, Il-Young Jung, $\mathrm{MD}^{7}$, Ueon Woo Rah, $\mathrm{MD}, \mathrm{PhD}^{8}$, \\ Byung Ok Kim, MD, $\mathrm{PhD}^{9}$, Kyoung Hyo Choi, MD, $\mathrm{PhD}^{10}$, Bum Sun Kwon, MD, PhD ${ }^{11}$, \\ Seung Don Yoo, $\mathrm{MD}, \mathrm{PhD}^{12}$, Heui Je Bang, $\mathrm{MD}^{6}$, Hyung-Ik Shin, $\mathrm{MD}, \mathrm{PhD}^{13}$, Yong Wook Kim, $\mathrm{MD}, \mathrm{PhD}^{14}$, \\ Heeyoune Jung, $\mathrm{MD}^{15}$, Eung Ju Kim, MD, $\mathrm{PhD}^{16}$, Jung Hwan Lee, $\mathrm{MD}, \mathrm{PhD}^{17}$, In Hyun Jung, MD, $\mathrm{PhD}^{9}$, \\ Jae-Seung Jung, $\mathrm{MD}, \mathrm{PhD}^{18}$, Jong-Young Lee, $\mathrm{MD}, \mathrm{PhD}^{19}$, Jae-Young $\mathrm{Han}, \mathrm{MD}, \mathrm{PhD}^{20}$, \\ Eun Young Han, MD, $\mathrm{PhD}^{21}$, Yu Hui Won, MD, $\mathrm{PhD}^{22}$, Woosik Han, $\mathrm{MD}^{23}$, Sora Baek, MD, PhD ${ }^{24}$, \\ Kyung-Lim Joa, MD, PhD ${ }^{25}$, Sook Joung Lee, MD, $\mathrm{PhD}^{26}$, Ae Ryoung Kim, MD ${ }^{27}$, So Young Lee, $\mathrm{MD}^{21}$, \\ Jihee Kim, $\mathrm{MD}^{28}$, Hee Eun Choi, $\mathrm{MD}^{29}$, Byeong-Ju Lee, $\mathrm{MD}^{30}$, Soon Kim, $\mathrm{PhD}^{31}$
}

\begin{abstract}
${ }^{1}$ Department of Rehabilitation Medicine, Inje University School of Medicine, Sanggye Paik Hospital, Seoul; ${ }^{2}$ Division of Cardiology, Department of Medicine, Sungkyunkwan University School of Medicine-Heart Vascular Stroke Institute, Samsung Medical Center, Seoul; ${ }^{3}$ Department of Physical Medicine and Rehabilitation, Dong-A University College of Medicine-Regional Cardiocerebrovascular Center, Dong-A Medical Center, Busan; ${ }^{4}$ Department of Rehabilitation Medicine, Seoul National University Bundang Hospital, Seoul National University College of Medicine, Seongnam; ${ }^{5}$ Gyeonggi Regional Cardiocerebrovascular Center, Seongnam; ${ }^{6}$ Department of Rehabilitation Medicine, Chungbuk National University College of Medicine, Chungbuk National University Hospital, Chungbuk Regional Cardiocerebrovascular Center, Cheongju; ${ }^{7}$ Department of Rehabilitation Medicine, Chungnam National University College of Medicine-Daejeon Chungcheong Regional Cardiocerebrovascular Center, Chugnam National University Hospital, Daejeon; ${ }^{8}$ Department of Physical Medicine and Rehabilitation, Ajou University School of Medicine, Suwon; ${ }^{9}$ Department of Internal Medicine, Inje University School of Medicine, Sanggye Paik Hospital, Seoul; ${ }^{10}$ Department of Rehabilitation Medicine, Asan Medical Center, University of Ulsan College of Medicine, Seoul; ${ }^{11}$ Department of Rehabilitation Medicine, Dongguk University School of Medicine, Goyang; ${ }^{12}$ Department of Rehabilitation Medicine, Kyung Hee University College of Medicine, Seoul; ${ }^{13}$ Department of Rehabilitation Medicine, Seoul National University College of Medicine, Seoul National University Children's Hospital, Seoul; ${ }^{14}$ Department and Institute of Rehabilitation Medicine, Yonsei University College of Medicine, Seoul; ${ }^{15}$ National Traffic Injury Rehabilitation Hospital, Yangpyeong; ${ }^{16}$ Division of Cardiology, Department of Medicine, Korea University Guro Hospital, Korea University College of Medicine, Seoul; ${ }^{17}$ Namdarun Rehabilitation Clinic,
\end{abstract}

Received February 12, 2019; Accepted May 31, 2019

Corresponding author: Goo Joo Lee

Department of Rehabilitation Medicine, Chungbuk National University Hospital, 776 1(il)sunhwan-ro, Heungdeok-gu, Cheongju 28644, Korea. Tel: +82-43-269-7845, Fax: +82-43-269-6228, E-mail: rmdr29@cbnuh.or.kr

ORCID: Chul Kim (http://orcid.org/0000-0001-8223-2945); Jidong Sung (http://orcid.org/0000-0002-1006-5727); Jong Hwa Lee (http://orcid.org/00000003-2489-358X); Won-Seok Kim (http://orcid.org/0000-0002-1199-5707); Goo Joo Lee (http://orcid.org/0000-0002-8436-4463); Sungju Jee (http:// orcid.org/0000-0002-9400-9609); Il-Young Jung (http://orcid.org/0000-0001-8204-8195); Ueon Woo Rah (http://orcid.org/0000-0002-5667-160X); Byung Ok Kim (http://orcid.org/0000-0002-7920-2750); Kyoung Hyo Choi (http://orcid.org/0000-0001-9137-3889); Bum Sun Kwon (http://orcid. org/0000-0001-7755-435X); Seung Don Yoo (http://orcid.org/0000-0003-4513-2560); Heui Je Bang (http://orcid.org/0000-0001-5280-122X); HyungIk Shin (http://orcid.org/0000-0001-8805-3104); Yong Wook Kim (http://orcid.org/0000-0002-5234-2454); Heeyoune Jung (http://orcid.org/00000002-4649-3053); Eung Ju Kim (http://orcid.org/0000-0003-2322-6267); Jung Hwan Lee (http://orcid.org/0000-0003-2680-6953); In Hyun Jung (http:// orcid.org/0000-0002-1793-215X); Jae-Seung Jung (http://orcid.org/0000-0002-8848-4112); Jong-Young Lee (http://orcid.org/0000-0001-5737-4968); Jae-Young Han (http://orcid.org/0000-0003-1672-8875); Eun Young Han (http://orcid.org/0000-0002-4780-8862); Yu Hui Won (http://orcid.org/00000003-2007-9652); Woosik Han (http://orcid.org/0000-0003-4002-3154); Sora Baek (http://orcid.org/0000-0003-3404-6202); Kyung-Lim Joa (http:// orcid.org/0000-0002-3747-9831); Sook Joung Lee (http://orcid.org/0000-0002-6894-445X); Ae Ryoung Kim (http://orcid.org/0000-0003-3765-3024); So Young Lee (http://orcid.org/0000-0002-0433-4548); Jihee Kim (http://orcid.org/0000-0003-2758-124X); Hee Eun Choi (http://orcid.org/0000-00028753-929X); Byeong-Ju Lee (http://orcid.org/0000-0002-7250-8909); Soon Kim (http://orcid.org/0000-0001-7154-2809).

(c) This is an open-access article distributed under the terms of the Creative Commons Attribution Non-Commercial License (http://creativecommons.org/ licenses/by-nc/4.0) which permits unrestricted noncommercial use, distribution, and reproduction in any medium, provided the original work is properly cited. Copyright (C) 2019 by Korean Academy of Rehabilitation Medicine 
Yongin; ${ }^{18}$ Department of Thoracic and Cardiovascular Surgery, Anam Hospital, Korea University Medical Center, Seoul; ${ }^{19}$ Division of Cardiology, Department of Internal Medicine, Kangbuk Samsung Hospital, Sungkyunkwan University School of Medicine,

Seoul; ${ }^{20}$ Department of Rehabilitation Medicine, Gwangju-Jeonnam Regional Cardiocerebrovascular Center, Chonnam National University Medical School \& Hospital, Gwangju; ${ }^{21}$ Department of Rehabilitation Medicine, Jeju National University School of Medicine, Jeju National University Hospital, Jeju; ${ }^{22}$ Department of Physical Medicine and Rehabilitation, Research Institute of Clinical Medicine of Chonbuk National University-Biomedical Research Institute of Chonbuk National University Hospital, Jeonju; ${ }^{23}$ Department of Thoracic Surgery, Chungnam National University Hospital, Daejeon; ${ }^{24}$ Department of Rehabilitation Medicine, Kangwon National University School of Medicine-Kangwon Regional Cardiocerebrovascular Center, Kangwon

National University Hospital, Chuncheon; ${ }^{25}$ Department of Rehabilitation Medicine, Inha University Hospital, Incheon; ${ }^{26}$ Daejeon St. Mary's Hospital. College of Medicine, The Catholic university of Korea, Daejeon; ${ }^{27}$ Department of Rehabilitation Medicine, School of Medicine Kyungpook National University, Daegu; ${ }^{28}$ Department of Rehabilitation Medicine and Institute of Wonkwang Medical Science, Wonkwang University School of Medicine, Iksan; ${ }^{29}$ Department of Physical Medicine and Rehabilitation, Inje University Haeundae Paik Hospital, Inje University College of Medicine, Busan; ${ }^{30}$ Department of Rehabilitation Medicine, Pusan National University Hospital, Busan; ${ }^{31}$ Research Institute for Social Science, Ewha Woman's University, Seoul, Korea

Objective Though clinical practice guidelines (CPGs) for cardiac rehabilitation (CR) are an effective and widely used treatment method worldwide, they are as yet not widely accepted in Korea. Given that cardiovascular disease is the second leading cause of death in Korea, it is urgent that CR programs be developed. In 2008, the Government of Korea implemented CR programs at 11 university hospitals as part of its Regional Cardio-Cerebrovascular Center Project, and three additional medical facilities will be added in 2019. In addition, owing to the promotion of CR nationwide and the introduction of CR insurance benefits, 40 medical institutions nationwide have begun CR programs even as a growing number of medical institutions are preparing to offer CR. The purpose of this research was to develop evidence-based CPGs to support CR implementation in Korea.

Methods This study is based on an analysis of CPGs elsewhere in the world, an extensive literature search, a systematic analysis of multiple randomized control trials, and a CPG management, development, and assessment committee comprised of 33 authors-primarily rehabilitation specialists, cardiologists, and thoracic surgeons in 21 university hospitals and two general hospitals. Twelve consultants, primarily rehabilitation, sports medicine, and preventive medicine specialists, CPG experts, nurses, physical therapists, clinical nutritionists, and library and information experts participated in the research and development of these CPGs. After the draft guidelines were developed, three rounds of public hearings were held with staff members from relevant academic societies and stakeholders, after which the guidelines were further reviewed and modified.

Principal Conclusions CR involves a more cost-effective use of healthcare resources relative to that of general treatments, and the exercise component of CR lowers cardiovascular mortality and readmission rates, regardless of the type of coronary heart disease and type and setting of CR. Individualized CR programs should be considered together with various factors, including differences in heart function and lifestyle, and doing so will boost participation and adherence with the CR program, ultimately meeting the final goals of the program, namely reducing the recurrence of myocardial infarction and mortality rates.

Keywords Acute coronary syndrome, Cardiac rehabilitation, Mortality, Clinical practice guidelines, Secondary prevention

\section{INTRODUCTION}

Cardiovascular mortality rose by $42.8 \%$ over a 10 -year period in South Korea, to become the second leading cause of death in 2014. According to a 2016 report by Statistics Korea, of 100,000 people, 58.2 died from cardiovascular disease (CVD), and of these $52 \%$ died from ischemic heart disease, namely acute myocardial infarction (AMI) [1-3]. The high mortality rate is influenced by deaths at the time of onset as well as deaths from recurrence and complications in the short-term and long-term after onset. The disease is accompanied by multiple problems, such as reduced exercise capacity and poorer quality of life (QOL). 
Acute coronary syndrome (ACS) clinically manifests as an acute condition, but is actually an acute onset of chronic systemic vascular disease (atherosclerosis) that has been progressing slowly over a long period. Further, despite receiving appropriate treatment in the acute phase, patients are left with a chronic vascular condition, so lifelong treatment and management is essential following discharge [4]. Moreover, even after being treated for acute CVD, patients with tachycardia, frequent arrhythmia, reduced cardiac output, orthostatic hypotension, and exercise-induced hypertension have difficulty exercising, and older patients and those with complications face a permanent and marked reduction in their capacity to exercise at an appropriate fitness level. When this state persists, atherosclerosis progresses further, thereby causing adverse outcomes in the long-term-5 to 10 years after onset (e.g., QOL, recurrence, readmission, death). Therefore, patients should be put on a cardiac rehabilitation (CR) program as soon as they are stabilized following acute phase treatment. This program includes individualized exercise training, optimal medical treatment, and education for self-care of risk factors (e.g., smoking cessation, diet therapy, physical activities, diabetes and hypertension management, stress management).

CR is actively performed in many Western countries, where its effectiveness and safety have already been established, and clinical practice guidelines (CPGs) for CR have been developed in consideration of each country's circumstances, and medical and scientific evidence documenting CR has been systematically organized to inform medical professionals' decision-making [4-9].

In Korea, on the other hand, CVD treatment is still focused on acute phase treatment and lacks a sound understanding of CR. As a result, only a handful of hospitals nationwide offer CR. The purpose of this research is to develop CPGs that will promote CR in Korea. The CPGs proposed here are based on medical and scientific evidence, and are intended to inform medical decisionmaking. These guidelines should not be uniformly applied to all patients, and medical professionals must make final decisions in consideration of a patient's medical state and other circumstances. These CPGs do not limit a physician's practice, and neither can they be used to assess or review a physician's practice.

These CPGs address CR and secondary prevention pro- grams for male and female adult patients who have been admitted and treated for ACS and survived the acute phase treatment. They do not deal with primary prevention of CVD or CR among pediatric patients. Moreover, due to limited time and resources, this first edition does not cover heart failure, valvular heart diseases, congenital heart diseases, arrhythmia, or other heart and peripheral vascular deformities, as such contents will be developed in future revisions and updates. ACS includes AMI and unstable angina, and acute phase treatment includes intensive treatment at the coronary care unit (CCU), medical drug therapy, percutaneous coronary interventions (PCIs), and coronary artery bypass grafting (CABG) surgery.

More specifically, the purpose of these CPGs is to provide best practice guidelines for CR after ACS based on objective, scientific evidence, for physicians and other medical professionals (e.g., nurses, physical therapists, clinical nutritionists) responsible for CR of patients with ACS. Furthermore, these guidelines were developed in an attempt to minimize gaps between medical and scientific evidence and actual clinical practice, as well as gaps in CR treatments across hospitals and physicians, thereby ultimately contributing to the development of a universal, evidence-based CR program. These CPGs discuss the health benefits and risks of each individual patient requiring CR. Their ultimate objective is to improve patients' QOL by restoring their cardiopulmonary functions, reducing recurrence and readmission, and lowering mortality rates.

These are the first CPGs for CR to be developed in Korea. Some contents of the Scottish [5] guidelines for CR were adapted with additional references to other foreign CPGs for CR [4-9], and based on studies published between January 1, 2009 and April 30, 2018. However, these CPGs only deal with patients experiencing ACS, so in future they will have to be supplemented by including content for other heart diseases. We aim to revise these guidelines every 3-4 years, when a consensus is reached on the need for revisions in light of new knowledge.

\section{METHODS}

\section{Research framework}

The group that developed these CPGs was comprised of writers and consultants. Thirty-three authors, primar- 
ily rehabilitation specialists, cardiologists, and thoracic surgeons in 21 university hospitals and two general hospitals, were involved in the CPG management, development, and assessment committees. During the research and development process, opinions were garnered from 12 consultants including rehabilitation, sports, and preventive medicine specialists, CPG experts, nurses, physical therapists, clinical nutritionists, and library and information experts.

This research was based on an extensive literature review of $\mathrm{CR}$, the assessment of CR outcomes, exercise therapies appropriate for $\mathrm{CR}$, and educational components for secondary prevention. The key questions related to each of these aspects were selected during a process that involved all members of the CPG management and development committees, and finalized with reference to the latest foreign CPGs [4-9], in consultation with the CPG consultation committee. Individual guidelines were then developed in relation to circumstances in Korea. Key questions that had not been adequately analyzed in existing guidelines were developed de novo, and finalized in consultation with the CPG consultation committee in consideration of the circumstances in Korea.

To select foreign CPGs for adaptation, the Scottish (2017) [5], British (2013) [6], American (2011) [7], Canadian (2009) [4], and Japanese (2012) [8] CPGs for CR published within the past ten years were evaluated using accepted Appraisal Guidelines for Research and Evaluation II (AGREE II) [10]. Two investigators appraised one set of CPGs, and guidelines assigned an average 'total score for each domain' of 80 or greater (out of a score of 161) by two investigators, and a 'rigor of development' score of $70 \%$ or greater for each domain (out of a score of 56 ).

As summarized in Table 1, the Scottish (2017) [5] and British (2013) [6] CPGs were selected after being reviewed by the CPG management committees, and the Scottish Intercollegiate Guidelines Network (SIGN) CPGs [5], which had been published more recently, were chosen as the major guidelines for adaptation in this study. References regarding specific key questions and topics were taken from all five foreign CPGs [4-9].

Percentage score for each domain $(\%)=$

Total score for each domain - minimum score for each domain
$\times 100$

The key questions were used to inform the CPGs were primarily selected during a process that involved all members of the CPG management and development committees. The Scottish CPGs (SIGN 2017) [5] were used as the primary reference, but the English (NICE 2013) [6], American (AHA 2011) [7], Canadian (CACR 2009) [4], and Japanese (JCS 2012) [8] CPGs were also used for reference in relation to key questions not adequately addressed in the SIGN 2017 guidelines. Those developed de novo due to the lack of available references were as follows: "Is a submaximal exercise test, such as a 6 -minute walk test

Table 1. Appraisal of foreign clinical practice guidelines for cardiac rehabilitation with AGREE II

\begin{tabular}{lcccccc}
\hline Guideline (country) & $\begin{array}{c}\text { Reviewer } \\
\text { no\# }\end{array}$ & $\begin{array}{c}\text { Rigor of } \\
\text { development }\end{array}$ & $\begin{array}{c}\text { Rigor } \\
\text { percentage }\end{array}$ & $\begin{array}{c}\text { Total score for } \\
\text { each domain }\end{array}$ & $\begin{array}{c}\text { Overall } \\
\text { appraisal }\end{array}$ & Result \\
\hline SIGN 2017 (Scotland) & 1 & 50 & 83 & 130 & 6 & Selected \\
& 2 & 46 & & 125 & 6 & \\
\hline NICE 2013 (England) & 3 & 50 & 89 & 121 & 6 & Partial selection \\
& 4 & 56 & & 135 & 6 & \\
AHA 2011 (USA) & 5 & 37 & 67 & 90 & 5 & Partial selection \\
& 6 & 30 & & 89 & 4 & \\
\hline CACR 2009 (Canada) & 7 & 20 & 44 & 71 & 3 & Partial reference \\
\hline & 8 & 38 & & 99 & 4 & \\
JCS 2012 (Japan) & 7 & 26 & 34 & 70 & 3 & Partial reference \\
\hline
\end{tabular}

AGREE, Appraisal Guidelines for Research and Evaluation; SIGN, Scottish Intercollegiate Guidelines Network; NICE, National Institute for Health and Care Excellence; AHA, American Heart Association; CACR, Canadian Association of Cardiac Rehabilitation; JCS, Japanese Circulation Society. 
useful for cardiac rehabilitation?", "Is high-intensity interval training more effective than continuous moderateintensity training as an aerobic exercise?", and "Should policosanol be recommended as a food supplement?" (Table 2).

\section{Data sources}

To gather evidence to inform the development of the CPGs, PubMed was searched using the following key terms and search strategies: Search ('cardiac rehabilitation' [TIAB] OR 'cardiac rehabilitation' [MeSH]), Filters (Consensus Development Conference; Guideline; NIH; Practice Guidelines). In addition, PubMed was searched for guidelines using key words such as myocardial infarction (MI) as follows: Search ((rehabilitation or 'secondary prevention') and ('myocardial infarction' or 'acute coronary syndrome')), Filters (Consensus Development Conference; NIH; Guidelines; Practice Guidelines).

Studies published after publication of the latest foreign CPGs were primarily found in the following three databases: Cochrane Library (www.cochranelibrary.com), PubMed (www.ncbi.nlm.nih.gov/pubmed), and EMBASE (www.embase.com).

MeSH terms were used 'cardiac rehabilitation' in the Cochrane Library and PubMed, and Emtree terms 'heart rehabilitation' were used when searching EMBASE. Based

Table 2. Key questions for clinical practice guidelines for cardiac rehabilitation

\begin{tabular}{|c|c|}
\hline Question & Description \\
\hline KQ1 & Must cardiac rehabilitation be included in the treatment of acute coronary syndrome? \\
\hline KQ2 & Does cardiac rehabilitation influence the outcome of patients with cardiovascular disease? \\
\hline KQ3 & Does cardiac rehabilitation improve the quality of life of patients with cardiovascular disease? \\
\hline KQ4 & When should cardiac rehabilitation be begun? (Timing of cardiac rehabilitation) \\
\hline KQ5 & How should cardiac rehabilitation programs be structured? \\
\hline KQ6 & $\begin{array}{l}\text { Can cardiac rehabilitation programs lower health management costs for patients with acute coronary } \\
\text { syndrome? }\end{array}$ \\
\hline KQ7 & $\begin{array}{l}\text { Are individualized cardiac rehabilitation programs more effective than the existing fixed cardiac } \\
\text { rehabilitation program? }\end{array}$ \\
\hline KQ8 & $\begin{array}{l}\text { Should psychological interventions concerning anxiety, depression, and stress be included in the } \\
\text { cardiac rehabilitation program? }\end{array}$ \\
\hline KQ9 & Is a cardiopulmonary exercise test necessary for cardiac rehabilitation? \\
\hline KQ10 & Is a submaximal exercise test, such as a 6-minute walk test, useful for cardiac rehabilitation? \\
\hline KQ11 & What are the effective measures for promoting participation in cardiac rehabilitation? \\
\hline KQ12 & What are the effective measures for increasing physical activity compliance rates? \\
\hline KQ13 & When should patients begin cardiac rehabilitation following coronary artery bypass grafting? \\
\hline KQ14 & Should aerobic exercises be included in the cardiac rehabilitation program? \\
\hline KQ15 & Should resistance (muscle training) exercises be included in the cardiac rehabilitation program? \\
\hline KQ16 & How can the safety of cardiac rehabilitation exercises be enhanced? \\
\hline KQ17 & $\begin{array}{l}\text { Can a home-based cardiac rehabilitation program replace a hospital-based cardiac rehabilitation } \\
\text { program? }\end{array}$ \\
\hline KQ18 & Should cardiac rehabilitation programs be recommended to elderly patients? \\
\hline KQ19 & Is patient education a necessary part of cardiac rehabilitation? \\
\hline KQ20 & What contents should be included in patient education? \\
\hline KQ21 & What interventions are needed to improve patients' adherence to taking their medications? \\
\hline KQ22 & What is an effective intervention for patients who need to stop smoking? \\
\hline KQ23 & What diet therapies are recommended for patients undergoing cardiac rehabilitation? \\
\hline KQ24 & Should a specific food supplement be recommended? \\
\hline KQ25 & Would an ICT-based modality be helpful in maintaining the effects of education in the long-term? \\
\hline
\end{tabular}

ICT, information \& communication technology. 
on the search strategies used in SIGN 2017, CR-related search strategies for each study question were developed, and the specific search terms for each were entered using AND or OR operators. Operators and synonyms were taken from the search strategies published in Cochrane Review articles found in the Cochrane Library using the MESH term 'cardiac rehabilitation'. After conducting a search in the Cochrane Library, the search strategy was saved, and the same search strategy used in the EMBASE. The Cochrane Library and EMBASE use the same search strategies, such as proximity operators, and the search strategy for PubMed was also nearly identical, with the exception of different proximity operators. Therefore, due to space limitations, we present only the search strategy used for the Cochrane Library in this report (refer to Appendix). When making revisions with reference to existing guidelines, we limited the searches to systematic review articles, and the year of publication was set to the year following the publication of the existing guidelines. Korean articles were searched in RISS, KMbase, and KoreaMed using terms 'cardiac rehabilitation' and '심장재 활' (cardiac rehabilitation). After the duplicate removal, the two researchers selected the first, second, and third document selection for the same documents. In case of disagreement during the article selection process, the consensus was reached after the discussion or the final decision was made by the third researcher's arbitration.

For assessing the methodological quality of article, AMSTAR 2.0, GRADE system used for systematic review, Cochrane risk-of-bias (RoB) used for randomized controlled trials, and the RoB assessment tool for non- randomized studies (RoBANS) used for non-randomized controlled trials. Two researchers evaluated the quality of one article. In case of disagreement during the assessing the methodological quality of article, the consensus was reached after the discussion or the final decision was made by the third researcher's arbitration. Data obtained in the search were accepted as evidence of the studies' populations (human cases of ACS), designs, and methods (sample size, blinding, data analysis), results (objectivity of results and consistency throughout studies), language (Korean and English), and applicability to Korea. Both adaptation and de novo methods were used. For the former, all available evidence cited in foreign guidelines was reviewed, as the level of evidence and recommendations presented by each country used different evidence appraisal methods, and an evidence chart was developed based on all the subsequent evidence published after the publication of the latest foreign CPGs. After drafting the CPGs, the levels of evidence (LOE) and strength of recommendations (SOR) were determined based on the evidence chart and with reference to the method used in the SIGN 2017 [5].

LOE ranged from 1++ (highest quality) to 4 (lowest quality) [5] (Table 3). The SOR was determined in consideration of the LOE, reliability and consistency of the LOE, impact on patients, applicability in Korea, and balance between harm and benefits, but instead of following the GRADE classification of ABCD, recommendations were classified as either strong or conditional and Good-Practice Points (GPPs) were given according to the method used in the SIGN 2017 (Table 4). The SIGN stopped using

Table 3. Levels of evidence [5]

\begin{tabular}{|c|c|}
\hline Level & Evidence statement \\
\hline $1++$ & $\begin{array}{l}\text { High-quality meta-analyses, systematic reviews of randomized control trials, or randomized control } \\
\text { trials with a very low risk of bias }\end{array}$ \\
\hline $1+$ & Well-conducted meta-analyses, systematic reviews, or randomized control trials with a low risk of bias \\
\hline $1-$ & Meta-analyses, systematic reviews, or randomized control trials with a high risk of bias \\
\hline $2++$ & $\begin{array}{l}\text { High-quality systematic reviews of case-control or cohort studies } \\
\text { High-quality case-control or cohort studies with a very low risk of confounding or bias and a high } \\
\text { probability that the relationship is causal }\end{array}$ \\
\hline $2+$ & $\begin{array}{l}\text { Well-conducted case-control or cohort studies with a low risk of confounding or bias and a moderate } \\
\text { probability that the relationship is causal }\end{array}$ \\
\hline 2- & $\begin{array}{l}\text { Case-control or cohort studies with a high risk of confounding or bias and a significant risk that the } \\
\text { relationship is not causal }\end{array}$ \\
\hline 3 & Non-analytic studies (e.g., case reports, case series) \\
\hline 4 & Expert opinion \\
\hline
\end{tabular}


Table 4. Recommendations and Good-Practice Points [5]

\begin{tabular}{l} 
Some recommendations can be made with more certainty than others. The wording \\
used in the recommendations in this guideline denotes the certainty with which the \\
recommendation is made (the 'strength' of the recommendation). \\
The 'strength' of a recommendation takes into account the quality (level) of the \\
evidence. Although higher-quality evidence is more likely to be associated with \\
strong recommendations than lower-quality evidence, a particular level of quality \\
does not automatically lead to a particular strength of recommendation. \\
Other factors that are taken into account when forming recommendations include: \\
relevance to the NHS in Scotland; applicability of published evidence to the target \\
population; consistency of the body of evidence, and the balance of benefits and \\
harms of the options. \\
R $\begin{array}{l}\text { For 'strong' recommendations on interventions that 'should' be used, the } \\
\text { guideline development group is confident that, for the vast majority of people, } \\
\text { the intervention (or interventions) will do more good than harm. For 'strong' } \\
\text { recommendations on interventions that 'should not' be used, the guideline } \\
\text { development group is confident that, for the vast majority of people, the } \\
\text { intervention (or interventions) will do more harm than good. } \\
\text { For 'conditional' recommendations on interventions that should be 'considered', } \\
\text { the guideline development group is confident that the intervention will do } \\
\text { more good than harm for most patients. The choice of intervention is therefore } \\
\text { more likely to vary depending on a person's values and preferences, and so the } \\
\text { healthcare professional should spend more time discussing the options with } \\
\text { the patient. } \\
\text { Recommended best practice based on the clinical experience of the guideline } \\
\text { development group. }\end{array}$ \\
Good-Practice Points (GPP) \\
\hline
\end{tabular}

the ABCD classification in 2013 due difficulties associated with its practical application, and has been classifying recommendations into strong, conditional, and GPPs since then [5].

\section{Process followed to develop cPGs}

Expert panels consisting of 11 rehabilitation specialists, cardiologists, and thoracic surgeons achieved formal consensus using the nominal group technique. Authors of the draft guidelines first reviewed the evidence collected with these panels, and then conducted a question and answer session, after which the final recommendations and the LOEs and SORs were determined through a vote. Participants gave their opinions using a 9-point scale (1, 2, 3 'inappropriate'; 4, 5, 6 'uncertain'; 7, 8, 9 'appropriate'). If the ratings given by more than $70 \%$ of the panel (8 out of 11) were within a single 3-point band, and there were no objections with the phrasing of the recommendation, a consensus was deemed to have been reached.

The SORs were indicated as strong, conditional, or GPPs, in consideration of the LOE, likely impacts on patients, their applicability in Korea, and the balance that would be achieved between benefits and harm. 'Strong' recommendations were used when it was deemed that the benefits of the recommendation would outweigh the harm for a vast majority of people. If recommended, it was phrased as 'must be performed, 'should be performed', or 'should be strongly recommended. If it was not recommended, the possible phrases used were 'must not be performed, 'should not be performed', and 'should not be recommended. The word 'conditional' was used when the benefits of the recommendation would outweigh its harms on most people. If recommended, it was phrased as 'recommend' or 'is recommended', and if it was not recommended, it was phrased as 'should not be performed' and 'is not recommended. Therefore, for interventions that were conditionally recommended, performance should be determined on the basis of an individual's values and preferences, and after discussing the intervention with the patient and ensuring the decision is based on a thorough understanding of the intervention's importance. Finally, GPPs were used when the SORs could not be determined due to a low LOE in the literature but the practice was deemed to be a recommended 
practice based on the guideline development group's clinical experience. It was phrased as 'is considered' and 'should be referred to'.

Staff from relevant academic societies and other persons concerned were invited to attend three rounds of public hearings on the draft guidelines (the Korean Academy of Rehabilitation Medicine, the Korean Society of Cardiology, and the Korean Society for Thoracic and Cardiovascular Surgery), following which they were reviewed and modified.

The CPG management committee designated one investigator responsible for each topic. These individuals then finalized the guidelines together with the CPG writing committee. The process of identifying key questions and preparing evidence summaries and meeting transcripts was used to reference the writing process. Internal revisions were made after the first review by the investigator in charge, and the CPG management committee then performed a final review. The CPG Committee at the Korean Academy of Medical Sciences was also asked to appraise the draft guidelines, and those comments will be taken into consideration in a future second revision.

Academic societies and associations for physicians caring for patients in CR will use the 2018 CPGs for CR in their professional development, as will health professionals in their consultations with patients and caregivers, and they will be used to inform various medical institutions' decisions related to convalescence. Following their distribution, the authors will continue to respond to questions and obtain opinions from health professionals using the CPGs. A second edition will be developed once consensus has been reached on the need for further revisions based on foreign CPGs and new evidence.

\section{LITERATURE REVIEW AND RESULTS}

This section reviews CR and secondary prevention programs for male and female adult patients admitted for ACS who survived after the acute phase treatment (e.g., ICU management, medical drug therapy, PCI, CABG). It considers in-hospital CR, the post-discharge CR process, and continuous risk factor management. It describes the rationale, timing, basic principles, and flow of $\mathrm{CR}$, before reviewing the various methods used to assess CR. Exercise therapy, the core component of CR, and the self-care of CVD risk factors needed for secondary prevention of
CVD conclude this section (Table 5).

\section{Introduction of cardiac rehabilitation}

Although patients with ACS risk death while being transferred to a hospital and during treatment after onset of the disease, many patients make dramatic recoveries

Table 5. Key recommendations of cardiac rehabilitation

1. Introduction of cardiac rehabilitation

- CR programs must be included in the treatment of ACS (SOR of strong / LOE of 1++)

- CR exercise should be initiated as early as possible following acute phase treatment (SOR of strong / LOE of 1-)

2. Assessments of cardiac rehabilitation

- Cardiopulmonary exercise testing should be performed to assess patients' cardiopulmonary exercise functions, prescribe exercise, and predict outcomes (SOR of strong / LOE of 2++)

- Interventions to increase self-efficacy and awareness of the need for CR are needed to increase CR participation (SOR of strong / LOE of 1++)

- Systematized automatic referrals and liaison systems should be considered to increase CR referral rates. (SOR of GPP / LOE of 2-)

3. Exercise therapy for cardiac rehabilitation

- CR should be initiated early after surgery, as an inpatient program for patients who undergo CABG (SOR of strong / LOE of 1++)

- Risk assessment and appropriate monitoring based on the risk assessment results should be performed to ensure patient safety during CR exercise programs (SOR of srong / LOE of 2++)

- CR programs should also be administered to patients aged 65 years and older (SOR of strong/ LOE of $1++$ )

4. Education for secondary prevention

- CR programs should include patient education (SOR of strong / LOE of 1++)

- Smoking cessation interventions should be provided for patients who smoke, and continuous interventions of more than 4 weeks should be considered (SOR of strong / LOE of 1++)

- ICT-based modalities should be considered to maintain the effects of education (SOR of conditional / LOE of 1-)

CR, cardiac rehabilitation; SOR, strength of recommendation; LOE, level of evidence; ICT, information \& communication technology. 
from the condition after treatment in the acute phase. Consequently, many patients, and even medical professionals, are excessively optimistic about the long-term prognosis and outcomes of this disease, and once the symptoms have abated after the acute phase treatment, many patients revert to the lifestyles they had adopted prior to disease onset, with a total disregard for risk factor management.

As reflected in their CPGs, many other countries have already accepted CR as an essential component of comprehensive treatment for ACS (LOE of 1++), including Scottish, British, and American guidelines [7,11-13]. Until recently, many studies analyzed the efficacy of CR in relation to CVD and patients' mortality, recurrence, admission risk, and QOL. Scotland's CPGs state that patients should be offered a CR program that includes an exercise component to reduce cardiovascular mortality, hospital readmissions, and improve patients' QOL (SIGN 2017; SOR strong and LOE of 1++) [5]. England's guidelines are that all patients (regardless of age) should be informed about and offered a CR program that includes an exercise component (NICE 2013) [6]. US guidelines state that all eligible patients with ACS, or patients' whose status is immediately post CABG surgery or post-PCI, should be referred to a comprehensive outpatient CR program, either prior to hospital discharge or during the first followup office visit (AHA 2011; SOR of I / LOE of A) [7].

The Cochrane Review (2016) summarized the findings of a systematic literature review and meta-analysis of patients with AMI, patients who underwent revascularization, such as CABG and PCI, patients with angina, and patients diagnosed with coronary heart disease (CHD) based on angiography. Exercise-based CR lowered cardiovascular mortality (risk ratio $[\mathrm{RR}]=0.74 ; 95 \%$ confidence interval [CI], 0.64-0.86) and risk for readmission (RR=0.82; 95\% CI, 0.70-0.96), but did not significantly reduce the risk of re-infarction (RR=0.90; 95\% CI, 0.79-1.04) or all-cause mortality (RR=0.96; 95\% CI, 0.88-1.04) (LOE of 1-) [14]. In a systematic review of eight observational studies on the effects of CR published after the year 2000, CR lowered the all-cause mortality (adjusted odds ratio $[\mathrm{OR}]=0.47,95 \% \mathrm{CI}, 0.38-0.59$ ) and cardiovascular mortality (adjusted OR=0.43; 95\% CI, 0.23-0.79) of patients with acute MI (LOE of 1+) [15]. CR was initially focused on exercise therapy, but has since transitioned to become a comprehensive program that includes mental sup- port, risk factor corrections, and education for lifestyle transformations. Behavioral change techniques (BCTs) include providing information about the outcomes of a behavior, educating patients on how to behave, encouraging outcome-related goal-setting, providing information about specific behaviors to be performed at specific times and places, providing feedback, and training patients how to manage stress and control their emotions. Compared to the control group, which only received the usual care, BCT interventions demonstrated better outcomes in smoking cessation ( $\mathrm{RR}=0.89 ; 95 \% \mathrm{CI}, 0.81-0.97$ ), with significantly lower mortality risk $(\mathrm{RR}=0.82$; $95 \% \mathrm{CI}$, 0.69-0.97), and $3.13 \mathrm{mmHg}$ lower SBP (95\% CI, -5.11 to -1.15). However, the CHD risk was not significantly reduced with BCTs (LOE of 1+) [16].

A Korean study also reported that a CR group (10\%, 7 of 69 patients) had significantly lower recurrence rates (AMIs, readmission rates, need for reperfusion, mortality rates) and significantly longer disease-free days (354 \pm 38.34 days vs. $316 \pm 99.96$ days) compared to the control group (24\%, 17 of 72 patients) [17].

Whether CR lowers mortality rates from heart disease is a critical question in relation to the need for CR. Although the results of recent studies on the effects of CR have been partially inconsistent, multiple studies have reported that the exercise component of CR lowers cardiovascular mortality and readmission rates, regardless of the type of CHD and type and setting of CR $[14,18,19]$.

Scotland's CPGs state that patients should be offered a CR program that includes an exercise component to reduce cardiovascular mortality, hospital readmissions, and improve their QOL, and note that the exercise component of CR reduces cardiovascular mortality and hospital admissions, and improves health-related QOL, regardless of the type of CHD, the type of $\mathrm{CR}$, or its setting (SIGN 2017; LOE of 1++) [5].

The 2016 Cochrane Review-an online collection of six databases that summarize high-quality, independent evidence to inform healthcare decision-making-summarized the results of a systematic literature review and meta-analysis of randomized controlled trials (RCTs) involving patients with AMI, patients who underwent revascularization, such as CABG and PCI, patients with angina, and patients diagnosed with CHD based on angiography. Exercise-based CR lowered cardiovascular mortality (RR=0.74; 95\% CI, 0.64-0.86) and risk for read- 
mission (RR=0.82; 95\% CI, 0.70-0.96), but did not significantly reduce the risk of all-cause mortality $(\mathrm{RR}=0.96$; 95\% CI, 0.88-1.04) (LOE of 1-) [14]. In another systematic literature review and meta-analysis of 31 studies of 6,926 patients published in 2017, over a 10-year follow-up, the comprehensive CR group had significantly lower cardiovascular mortality compared to that of the usual care group ( $R R=0.74 ; 95 \% \mathrm{CI}, 0.65-0.86 ; \mathrm{p}<0.0001$ ) (LOE of 1+) [20]. In a meta-analysis of 18 studies including 7,691 patients who underwent CABG or reperfusion after MI or were diagnosed with coronary stenosis based on coronary angiography, the CR group had similar all-cause mortality (RR=1.00; 95\% CI, 0.88-1.13), but significantly lower cardiovascular mortality compared to that of the control group (RR=0.42; 95\% CI, 0.21-0.88) (LOE of $1+$ ) [21].

Verifying whether CR improves patients' QOL following the onset of heart disease is key to patients' abilities to return to their daily lives. Recent studies on the effects of CR found that the exercise component of CR improves these individuals' QOL, regardless of the type of coronary syndrome and type and setting of CR $[14,18,19]$, and benefits the cardiopulmonary health and QOL of patients with heart failure with preserved ejection fraction $[22,23]$.

Scotland's CPGs state that patients should be offered a CR program that includes an exercise component to reduce cardiovascular mortality and hospital readmissions and improve patients' QOL. These benefits are known to occur regardless of the type of $\mathrm{CHD}$, the type of $\mathrm{CR}$, or the setting (SIGN 2017; LOE of 1++) [5].

Meta-analysis could not be performed in a systematic literature review of 63 RCTs that examined exercisebased CR in patients with coronary syndrome due to the heterogeneity of outcome measurement methods and reporting methods in 20 studies that assessed health-related QOL using verified general or disease-specific outcome measurement methods. Nonetheless, most of these studies found an improvement in the QOL of those in the CR group. Further, 14 studies reported a higher QOL in one or more domains in the exercise-based CR group compared to the control group, and 5 studies reported a significantly higher QOL in more than $50 \%$ of domains in the CR group compared to those in the control group (LOE of 1-) [14].

\section{RECOMMENDATION}

- CR programs must be included in the treatment of ACS (SOR of strong / LOE of 1++)

\section{Timing of cardiac rehabilitation}

The Scottish CPGs (SIGN 2017) state that "all patients must be referred to CR to undergo individualized intervention and treatment plans based on individualized assessment," [5] regardless of the type of treatment they undergo after the onset of ACS, and notes that their treatment should be comprehensive and individualized at the initiation of the rehabilitation program. There is not enough clinical evidence, however, to document the optimum timing of exercise, a critical component of CR, and early exercise is often determined passively. We therefore reviewed evidence regarding the timing of CR exercise in existing guidelines and additional studies.

US guidelines are that all eligible patients with ACS or patients' whose status is immediately post CABG surgery or post-PCI should be referred to a comprehensive outpatient CR program either prior to hospital discharge or during the first follow-up office visit. Further, all eligible outpatients with the diagnosis of ACS, CAB surgery or PCI, chronic angina and/or peripheral artery disease in the past year should be referred to a comprehensive outpatient CR program (AHA 2011; SOR of I / LOE of A) [7]. England's guidelines are that CR should be initiated as soon as possible after admission and before discharge from hospital. Patients should be invited to a CR session within 10 days of their discharge from hospital (NICE 2013) [6].

Haykowsky et al. [24] reported that commencing and maintaining exercise training early after the onset of MI (within 1 week of onset) leads to benefits in left ventricular (LV) remodeling (LOE of 1+). Most studies examining the timing of CR analyzed the effects of exercise training on LV remodeling, and found that exercise training in the acute phase or early phases since onset produces positive outcomes. These studies describe the timing of the exercise component of CR, as opposed to the timing of the CR per se. The risk of bias was low in clinical trials included in a 2016 meta-analysis that analyzed the impacts of the timing of exercise training on LV functions. These studies classified the timing of exercise training into an acute phase (6 hours to 7 days), a recovery phase ( 7 to 28 days), and a maintenance phase ( $\geq 29$ days), and examined dif- 
ferences in the left ventricle ejection fraction (LVEF), $\mathrm{LV}$ end-systolic diameter, and peak $\mathrm{VO}_{2}$ of patients with reduced LV function after MI. Although results on the changes of LVEF and end-systolic diameter varied widely, exercise training in the acute phase led to significant improvements (more than moderate improvements) in $\mathrm{LV}$ end-systolic diameter, and peak $\mathrm{VO}_{2}$ that were also greater than those produced by exercise training begun in the recovery phase and the maintenance phase. In conclusion, exercise training had positive effects on LV remodeling and recovery of cardiopulmonary functions, with the greatest effects achieved when exercise training was initiated in the acute phase (LOE of 1-) [25]. In this context, we determined that it is more appropriate to provide recommendations for the timing of exercise training as opposed to the timing of CR.

\section{RECOMMENDATION}

- It is more appropriate to provide recommendations for the timing of exercise training than the timing of CR

- CR exercise should be initiated as early as possible following acute phase treatment (SOR of strong / LOE of 1-)

\section{The organization of cardiac rehabilitation}

Because staff numbers, equipment, and facilities vary according to the size, capital, number of relevant medical professionals, and number of eligible patients in each institution, CR programs should be structured on basic concepts in consideration of organizational circumstances. The structure of a multidisciplinary team, and particularly the number of people on the team and the type of health care professionals, is directly dependent on the number of patients and contents of the program, the complexity of the patients being treated, the organization's human resources policies, and the community resources available to deliver the program [4].

Scotland's guidelines state that CR services should offer individualized exercise assessments, tailor the exercise components to individuals' choices, and deliver them in a range of settings (SIGN 2017; SOR strong) [5]. Canada's guidelines state that all CR programs must address sitespecific facilities' equipment in conjunction with safety requirements and considerations, and all CR programs must have policies and procedures to address the management of environmental and medical emergency situations (CACR 2009) [4].
In a systematic literature review of 8 observational studies that compared patients participating in a CR program with those not participating in a CR program, CR reduced all-cause and cardiac-related mortality rates, and significantly improved patients' health-related QOL [26]. More than half of these studies adopted a multidisciplinary team approach, and structured their teams to provide interventions regarding exercise education, healthrelated information and education, lifestyle modifications, mental health support, and social support (LOE of 1-). A meta-analysis of 71 RCTs that reported the effects of comprehensive CR in coronary artery diseases (CAD) found a reduction of all-cause and cardiac-related mortality, noncritical re-infarction and readmission rates, and also found that many types of professionals were involved in providing exercise and psychological treatments, risk factor education and counseling (LOE of 1-) [15]. In a systematic literature review analyzing 63 studies investigating the effects of exercise-based CR on CHD, CR lowered cardiovascular mortality rates and risk of hospital admission, and the rehabilitation programs included strategies for exercise and risk factor education, behavior change, psychological support, and traditional risk factor management (LOE of 1-) [14].

In 2010, the Korean Society of Cardiac Rehabilitation discussed and adapted various Korean and foreign CR guidelines, including the American Association of Cardiovascular and Pulmonary Rehabilitation (AACVPR) guidelines, and relevant studies that provided recommendations for the basic facilities, equipment, and personnel required in Korea's CR centers [27].

\section{RECOMMENDATION}

- CR programs should be comprehensive and multidisciplinary (SOR of GPP / LOE of 1-)

\section{Cost-effectiveness of cardiac rehabilitation}

The cost-effectiveness of CR has been reported in multiple studies [14]. These authors consistently concluded that CR involves a more cost-effective use of healthcare resources relative to that of general treatments. This conclusion is based on foreign literature however, so high quality Korean studies are needed to assess the costeffectiveness of CR in Korea, based on the total cost of healthcare and the cost per quality-adjusted life year (QALY) analysis. 
Scotland's guidelines state that interventions are considered to be cost effective if they fall below the commonly accepted UK threshold of $£ 20,000$ per QALY. However, no evidence of the cost-effectiveness of CR was identified in the UK, and additional higher-quality trials are needed to assess the cost-effectiveness of CR (SIGN 2017; SOR conditional) [5].

According to a systematic literature review analyzing 63 RCTs involving exercise-based CR in patients with coronary syndrome, it was difficult to compare 7 studies on the cost of CR and overall health management costs due to differences in their currencies and study periods, and given that 4 studies used a QALY that ranged from $\$ 42,535$ higher to $\$ 650$ lower per QALY gained in the CR group compared to the control group (LOE of 1-) [14].

\section{RECOMMENDATION}

- Though CR programs are reported to lower health management costs in patients with CVD, high-quality Korean studies are needed to substantiate this, considering the differences of insurance systems and healthcare environments across countries (SOR of GPP / LOE of 1-)

\section{Assessments of cardiac rehabilitation}

Use individualized assessments to develop care plans and interventions

Patients referred to CR following MI are in diverse medical, social, and financial situations. Therefore, individualized CR programs should be considered together with various factors, including differences of heart function such as cardiac output, comorbidities that hinder exercise, such as lung disease, arthritis, and low back pain, differences in lifestyle such as smoking and drinking status, and patients' ability to visit the hospital for CR according to employment status and distance from home. This is crucial to boosting participation and adherence with the CR program and ultimately achieving its goal, namely reducing the recurrence of $\mathrm{MI}$ and mortality rates. For this reason, the British Association for CR describes an individualized assessment of patient needs as an important component in the early phase of CR [28].

Scotland's guidelines state that all patients referred to CR should undergo an individualized assessment that is used to create to a care plan and identify interventions specific to their needs. In addition, CR services should offer individualized exercise assessments, tailor the exercise components of their programs to individual choice, and deliver them in a range of settings (strong) (SIGN 2017; SOR of GPP, strong / LOE of 1+) [5].

In a prospective cohort study that compared a general CR and an individualized CR program, participation was about $30 \%$ higher in the individualized CR program ( $\mathrm{p}<0.0001)$, and the risk for admission was significantly lower with the individualized CR program $(\mathrm{RR}=0.664$; 95\% CI, 0.554-0.797) (LOE of 2+) [29]. A Cochrane Review of 19 RCTs $(n=10,856)$ examined the effects of programs that consisted of goal setting and action planning based on individualized assessments of various chronic diseases, such as heart failure, kidney failure, and diabetes, and found that individualized programs were more effective than those of the control group in lowering glycated hemoglobin (-0.24\%; 95\% CI, -0.35 to -0.14$)$ and systolic blood pressure (-2.64 mmHg; 95\% CI, -4.47 to -0.82$)$, and improving depression (standardized mean differences [SMD] $=-0.36 ; 95 \% \mathrm{CI},-0.52$ to -0.20$)$ and self-efficacy (SMD $=0.25$; 95\% CI, 0.07-0.43) (LOE of 1+) [30]. In a RCT of 132 patients admitted for a heart disease such as CAD, a higher percentage of patients agreed to be referred to a lifestyle adjustment program in the individualized assessment group than in the conventional assessment group (27\% vs. $5 \%$ ) (LOE of $1+$ ) [31]. In a RCT of 40 patients with ACS, the intervention group, members of which received educational components according to their needs at an early stage, showed significantly higher self-efficacy compared to those in the control group, and a higher percentage of the intervention group participated in more than $90 \%$ of outpatient CR sessions ( $47 \%$ vs. $21 \%$ ) (LOE of $1+$ ) [32]. In a RCT of patients with ACS, providing individualized goals and plans $(n=78)$ led to a high improvement in outcome indices that included self-efficacy, return to work or the recovery of pre-onset functions, readmission, and mortality, compared to the conventional method $(\mathrm{n}=105)(21.8 \%, \mathrm{n}=17$ vs. $10.5 \%, \mathrm{n}=11 ; \mathrm{p}=0.039)$ [33].

\section{RECOMMENDATION}

- Individualized CR plans should be tailored to individual needs and be developed through individual assessments of patients referred to CR (SOR of strong / LOE of 1+) 


\section{Psychological evaluations and interventions}

Psychological symptoms such as depression, anxiety, and stress are common among patients with MI, and these symptoms can lead to recurrence, increased mortality, and reduced QOL. At about $15 \%$ to $20 \%$, the prevalence of depression among patients with $\mathrm{MI}$ is about three times higher than that of the general population $[34,35]$, and similar prevalence rates are observed among patients who have undergone PCI or CABG [36]. Depression undermines secondary prevention by lowering adherence with treatment plans, such as drug therapies, lifestyle adjustments, and CR participation [37-39], and may increase medical costs [40] for patients with heart disease. Anxiety and stress have been associated with recurrence and mortality rates among patients with $\mathrm{CAD}$ $[41,42]$. For this reason, psychological assessments and interventions have recently been recognized as essential components of CR programs.

Scotland's guidelines are that all patients should be offered a psychological care package, based on a cognitive behavioral model (e.g., stress management, cognitive restructuring, and communication skills) as an integral part of CR (SIGN 2017; SOR of strong / LOE of 1++) [5]. US guidelines are that patients with recent CABG surgery or MI should be screened for depression, in collaboration with their primary care physicians and a mental health specialist (AHA 2011; LOE of B) [7]. Canada's guidelines are that all CR patients should undergo screening for active and historical depression and anxiety at their intake assessment, and a qualified professional such as a psychologist or psychiatrist should refer individuals who screen positive for depression or anxiety for assessment or treatment (CACR 2009) [4]. Japan observes that because the prevalence of depression and depressed states is high among patients with CAD, and depression has been reported to relate to increased morbidity and mortality from CD, so specialists should screen for and treat depression in this patient population. Screening for depressed moods or other psychological symptoms, as well as conducting psychological interventions for the prevention and treatment of depression, are expected to alleviate psychological symptoms, improve QOL, enhance adherence to treatment, and reduce the mortality and incidence of cardiovascular events (JCS 2012; LOE of $\mathrm{B}, \mathrm{C})[8]$.

A 2017 Cochrane meta-analysis reviewed 35 RCTs $(\mathrm{n}=10,703)$ with regard to the psychological interventions conducted for $\mathrm{CAD}$, and found that they did not reduce all-cause mortality, revascularization, or incidence of nonfatal MI compared to conventional treatment, but they did significantly lower heart disease-related mortality rates ( $\mathrm{RR}=0.79 ; 95 \% \mathrm{CI}, 0.63-0.98)$, and improved perceived depression ( $\mathrm{SMD}=-0.27 ; 95 \% \mathrm{CI},-0.39$ to 0.15 ), anxiety (SMD $=-0.24 ; 95 \% \mathrm{CI},-0.38$ to -0.09 ), and stress levels (SMD=-0.56; 95\% CI, -0.88 to -0.24 ) (LOE of $1++$ ) [43]. In a meta-analysis of 13 studies of the outcomes of psychological interventions in CVD patients, they were effective in reducing stress (Hedges' g effect size $=0.34$; $95 \%$ CI, -0.19 to 0.87 ), anxiety (effect size $=1.04 ; 95 \%$ CI, $0.53-1.54$ ), and depression (effect size $=0.67$; 95\% CI, 0.410.92) (LOE of 1+) [44]. A RCT that compared early counseling focused on psychological trauma and conventional stress counseling in 190 patients with ACS, found no significant differences in psychological symptoms, mortality rates, or CVD-related readmission rates between the two groups (LOE of 1-) [45].

\section{RECOMMENDATION}

- Patients referred to CR should be assessed for psychological problems, such as anxiety, depression, and stress, and should be provided with psychological interventions if abnormal findings are observed (SOR of strong / LOE of 1++)

\section{Symptom-limited cardiopulmonary exercise test}

The symptom-limited cardiopulmonary exercise test that provides useful information related to exercise prescriptions can be obtained by monitoring heart rate (HR), blood pressure (BP), symptoms, electrocardiography (ECG), and gas analysis, while gradually increasing exercise load [46]. Assessing the risk of participation in exercise-induced CR programs based on changes in HRs, abnormal ECGs, changes in BP, and the onset of symptoms as a result of increasing exercise load can be used to develop safe and effective exercise prescriptions [47]. Aerobic exercise capacity, as measured with $\mathrm{VO}_{2 \max }$, was associated with CVD patients' prognosis, and the effectiveness of CR can also be evaluated on the basis of its improvement after CR. Thus, in the absence of contraindications, the symptom-limited cardiopulmonary test is recommended for $\mathrm{CR}[7,8,47]$.

US guidelines recommend a risk assessment with a 
physical activity history and/or an exercise test for all patients, to guide their prognosis and prescription (AHA 2011; SOR of I / LOE of B) [7]. Canada recommends that a directly supervised graded exercise test (GXT) be part of the initial CR assessment prior to the initiation of therapy (CACR 2009) [4]. Japan recommends using a symptomlimited exercise stress test on day 14-21 after onset to predict prognosis, prescribe exercise training programs, and assess the treatment efficacy of CR (JCS 2012; LOE of B) [8].

Symptom-limited cardiopulmonary exercise testing is currently accepted as a standard in CR programs for patients after ACS, so it is difficult to conduct a new RCT to directly compare the clinical outcomes of patients who undergo this testing and those who do not. Consequently, we could not find such clinical trials in our search. However, large cohort studies on CVD patients found that $\mathrm{VO}_{2 \max }$ measured with cardiopulmonary exercise testing or exercise testing-based risk score are strongly correlated with all-cause mortality or heart disease-related mortality with consistent dose-response correlations. This suggests that maximal cardiopulmonary exercise testing is a useful tool for predicting the outcomes and risks during exercise for patients with ACS (LOE of 2++) [44]. In a study of 100 patients who underwent coronary angioplasty due to MI, patients were randomly divided into an exercise testing group $(n=50)$ and a non-exercise testing group $(n=50)$; physicians permitted seven out of 11 activities, including the return to work, earlier for the exercise testing group than for the non-exercise testing group (LOE of 1-) [48].

\section{RECOMMENDATION}

- Cardiopulmonary exercise testing should be performed to assess patients' cardiopulmonary exercise functions, prescribe exercise, and predict outcomes (SOR of strong / LOE of 2++)

\section{Submaximal exercise test (6-minute walk test)}

The symptom-limited exercise test is recommended to assess exercise capacity and risk, prescribe exercise, and predict outcomes for patients undergoing CR $[7,8,46]$. However, exercise, ECG, gas analysis equipment, and adequate space are needed to conduct this standard cardiopulmonary exercise testing, and the associated costs hinder the universal application of this testing across all medical institutions [49]. Where maximal exercise testing is difficult, a submaximal exercise test, such as a 6-minute walk test, is commonly recommended [50,51], and a 6 -minute walk distance is used to set exercise intensity and evaluate the effectiveness of CR [52,53]. In Korea, the 6 -minute walk test is generally used as a submaximal exercise test, so recommendations in consideration of the validity, reliability, and responsiveness of the 6-minute walk test are needed.

Japan's guidelines state that a submaximal exercise test conducted on day 4 after onset or thereafter should be used to predict prognosis, prescribe exercise-training programs, and assess treatment efficacy (JCS 2012; LOE of B) [8].

A 2012 systematic review of studies of the 6-minute walk test included six studies on validity, one study on reliability, and 11 studies on responsiveness [54]. The maximum metabolic equivalents (METs) obtained from the 6-minute walk test and symptom-limited exercise test were more than moderately correlated, and the maximum heart rate obtained from the 6 -minute walk test and symptom-limited exercise test were also more than moderately correlated. Changes in reliability ranged from $2 \%$ to $8 \%$, but the intraclass correlation coefficient (ICC) was 0.97 , based on which level of evidence was evaluated to be moderate. In a meta-analysis of studies on the responsiveness of the 6-minute walk test in $\mathrm{CR}$, the mean difference of a 6-minute walk distance before and after treatment was $60.43 \mathrm{~m}$ (95\% CI, 54.57-66.30; p<0.001), indicating a high responsiveness (LOE of $2+$ ). In a subsequent study, a repeated 6-minute walk test within 1 week after onset of MI had high reliability (ICC $=0.879$; $95 \%$ CI, 0.785-0.939), and the ICC for HR, rate of perceived exertion (RPE), measured using the Borg scale, and BP ranged from 0.880 to 0.934 , also showing high reliability (LOE of 2+) [55]. Hanson et al. [56] found a high reliability for the 6 -minute walk test (ICC $=0.94$ ), but the 6 -minute walk distance tended to increase with subsequent tests, suggesting the need to consider this fact when assessing treatment responsiveness (LOE of 2+). A moderate correlation between 6 -minute walk distance and $\mathrm{VO}_{2 \max }$ was found in a recent study of 49 patients referred to $\mathrm{CR}$ $(\mathrm{r}=0.56, \mathrm{p}<0.01)(\mathrm{LOE}$ of $2++)$ [57].

\section{RECOMMENDATION}

-A submaximal exercise test, such as a 6 -minute walk 
test, should be considered when symptom-limited exercise testing is difficult (SOR of conditional / LOE of 2+)

\section{Strategies to improve cardiac rehabilitation participa- tion}

Despite the effectiveness of CR in patients with ACS, the problem of low CR participation lingers. In the US, CR participation among patients with $\mathrm{MI}$ is about $35 \%$, and $20 \%-30 \%$ in Europe, while that in Japan is low, at about 4\%-8\% [58]. A recent Korean study also found a hospitalbased CR participation rate of about $30 \%$ among patients with ACS in three university hospitals, and various factors that included distance, cost, time, comorbidity, and perceptions of CR were identified as hindering factors [59]. Therefore, effective strategies that could increase early uptake or participation in and adherence to CR are essential. In the United States, the Million Heart Project has been undertaken to lower recurrence, readmission, mortality, and medical costs among patients with ACS by increasing their CR participation based on grounds for effective strategies [60].

Scotland's guidelines are that interventions to improve self-efficacy should be considered for inclusion in a CR program. Further, the strategies associated with increased uptake in at least one trial were: (1) structured telephone calls/visits by a nurse/therapist after hospital discharge; (2) early appointments to CR; (3) motivational letters based on the theory of planned behavior; (4) CR programs tailored for women; and (5) intermediate phase programs (self-management, instruction, and exercise monitoring) for older patients (SIGN 2017; SOR of conditional / LOE of 1++) [5]. Canada recommends maximizing the number of patients who may benefit from CR programs by developing systematized, preferably automated, referral mechanisms that are sensitive to socioeconomic and ethno-cultural diversity with key patient care partners and other CVD stakeholders. An automated referral process should be used to significantly increase referrals from acute care to CR and subsequent enrollment (CACR 2009) [4].

A 2014 Cochrane Review of RCTs on CR participation among CVD patients included 10 studies on early CR participation $(n=1,338)$ and eight studies on CR maintenance $(\mathrm{n}=1,167)$, but a meta-analysis could not be performed due to the varying types of treatment involved [61]. Eight out of 10 studies and 3 out of 8 studies, however, found a significant increase in CR participation and maintenance, respectively. Strategies that increased CR participation included structured phone interviews or house visits after discharge [62-65], early visits to rehabilitation programs, [66] and letters that recommended CR participation [67]. Three studies that observed an increase in CR maintenance rates used physical monitoring based on daily journals, goal setting, and execution plans as strategies (LOE of 1++) [68-70]. In a RCT of 141 patients with ACS, physical activity rates increased in the intervention group that received motivating counseling compared to the control group (LOE of 1+) [71]. In a RCT that compared a female-only CR program and a mixed-gender $\mathrm{CR}$ program, there were no significant differences in participation rates between the two groups (LOE of 1++) [72]. In a RCT of 825 patients with ischemic heart disease or heart failure, participants were divided into two groups, one of which received instructions on learning and coping strategies and another that participated in conventional CR program strategies. The results showed that a higher percentage of the former group participated in more than $75 \%$ of the CR sessions ( $80 \%$ vs. $73 \%$; OR $=1.48 ; 95 \%$ CI, 1.07-2.05) (LOE of 1+) [73].

In a systematic literature review of 14 observational or intervention studies on the use of systematized automatic referrals and a liaison system to boost CR participation, meta-analysis revealed that the CR participation rate was highest when both systematized automatic referrals and a liaison system were applied (66\%; $95 \%$ CI, $54 \%-77 \%)$, as opposed to using only the conventional CR referral system (24\%; 95\% CI, $18 \%-32 \%)$, only the systematized automatic referral system (45\%; $95 \% \mathrm{CI}, 33 \%-57 \%)$, or only the liaison system (44\%; 95\% CI, 35\%-53\%) (LOE of 2-) [74].

\section{RECOMMENDATION}

- Interventions to increase self-efficacy and awareness of the need for CR are needed to increase CR participation (SOR of strong / LOE of 1++)

- Systematized automatic referrals and liaison systems should be considered to increase CR referral rates (SOR of GPP / LOE of 2-)

\section{Strategies to maintain long-term physical activities}

Maintaining physical activities is important to reducing CVD recurrence rates and improving patients' QOL in the 
long-term, after undergoing exercise-based CR. Although fitness is improved and maintained for a certain amount of time after participating in a CR program [75], these effects are often not translated into maintaining physical activities in the long-term $[76,77]$. In a Korean study administering physical activity surveys before and after the onset of MI, 37\% of the patients who were active prior to MI became inactive after onset, and $11 \%$ of the patients who were inactive prior to MI maintained physical activities after onset, thereby suggesting the need for strategies to promote the maintenance of physical activities in the long-term [78].

Scotland's guidelines are that psychoeducation (goal setting, self-monitoring) should be considered for patients in CR to facilitate adherence to physical activities, and noted that the interventions reported to be of benefit were: (1) goal setting, (2) action planning, and (3) using daily diary entries to monitor activities (SIGN 2017; SOR of conditional / LOE of 1++) [5].

In a meta-analysis of 209 patients in four studies, psychological education (using strategies such as goal setting, problem solving, and self-monitoring) led to greater long-term physical activities compared to those promoted by simple education about exercise and risk factors (SMD=0.62; 95\% CI, 0.30-0.94) (LOE of $1++$ ) [79]. In a systematic literature review of strategies used to maintain physical activities in the long-term, one study examined the effects of a problem-based learning strategy, but the study failed to find any significant effects (LOE of 1+) [80]. Further, a study on improving self-efficacy also did not find significant differences [81], while one RCT that used self-monitoring observed significant effects [82]. In a recent RCT of 47 patients with CVD, no significant differences in the maintenance of physical activities 1 year after the program were found between a voluntary, incremental physical activity program and the existing supervised exercise program (LOE of 1+) [83]. However, the addition of group education regarding exercise based on the use of a pedometer and face-to-face counseling to the conventional CR program significantly increased the duration of patients' continuous moderate to vigorous exercise 1 year after the program (LOE of 1+) [84].

\section{RECOMMENDATION}

- Psychological education strategies (including goal setting and self-monitoring) should be used to increase
CR maintenance rates (SOR of strong / LOE of 1++)

\section{Exercise therapy for cardiac rehabilitation}

Timing of cardiac rehabilitation in the case of coronary artery bypass surgery

Early CR is usually recommended if PCI is performed for ACS. In cases of CABG, CR may be delayed due to concerns about general deconditioning and the recovery of the sutured site after sternotomy. Thus, we propose appropriate timing for $\mathrm{CR}$ in cases involving $\mathrm{CABG}$ for ACS.

US guidelines recommend CR for all patients after CABG, with the referral ideally performed early postoperatively during the surgical hospital stay (AHA 2015; SOR of I / LOE of A) [85], and all eligible patients with ACS, or those whose status is immediate post-CAB surgery or post-PCI should be referred to a comprehensive outpatient CR program either prior to hospital discharge or during the first follow-up office visit (AHA 2011; SOR of I / LOE of A) [7].

Patients with ACS who underwent CABG and participated in CR showed significantly reduced mortality rates, CVD incidence, readmission rates, and length of hospital stays, compared to those in the control group $[86,87]$. According to AHA recommendations in 2015, CR is recommended for all patients who undergo CABG, and being referred to $\mathrm{CR}$ as an inpatient early after surgery is ideal [85]. Furthermore, according to AHA recommendations made in 2011, all patients who undergo CABG should begin CR while hospitalized, and must be referred immediately after discharge or at the first outpatient visit for a more comprehensive outpatient-based CR program [7].

A systematic review of nine randomized controlled studies on the effects of CR after CABG on patients with ACS found that the CR group showed improved quality of life and reduced cardiovascular risk factors [88]. However, this study could not systematically analyze the effects of specific rehabilitation regimens, since the type, intensity, and frequency of exercise varied in different CR programs across the studies, and diverse assessment parameters were used to analyze the effects of CR (LOE of 1-). It has been reported in Korea that beginning CR in the hospital after CABG leads to lower resting heart rates and increased 6-minute walking distances at the time of discharge and 1 month after surgery [89]. 


\section{RECOMMENDATION}

- CR should be initiated early after surgery, as an inpatient program for patients who undergo CABG (SOR of strong / LOE of 1++)

\section{Aerobic exercise program}

CR programs encompass the management of various risk factors that increase the risk of CVD. In particular, interventions for physical activities are a major component of CR programs [90]. Among various interventions for physical activities, we presented published evidence from aerobic exercise programs, and reviewed the different effects of CR according to the types of aerobic exercise involved.

Scotland's guidelines are that aerobic and resistance exercises should be considered part of the exercise prescription for patients attending CR (SIGN 2017; SOR of conditional / LOE of 1++) [5]. Canada recommends that patients in CR engage in aerobic and resistance exercise 3-5 times per week, at $40 \%-85 \%$ of their HR reserve, for 20-40 minutes per session. Each exercise session should include an appropriate warm-up and cool-down period. These guidelines further note that regardless of the form of interval training, there is compelling evidence that interval training can lead to improvements in peak $\mathrm{VO}_{2}$, functional status, and overall QOL. Accordingly, $\mathrm{CR}$ programs have increasingly used interval training as an alternative to traditional continuous aerobic training (CACR 2009) [4].

The exercise component of CR lowers cardiovascular mortality and readmission rates while increasing QOL [91]. Though the methods, frequencies, durations, and intensities of exercise vary widely across the studies conducted, nonetheless, studies have reported that CR is effective, regardless of specific frequency, duration, intensity, and place of exercise (LOE of $1++$ ) $[18,19,92]$. The SIGN 2017 recommends that aerobic exercise should be included in exercise prescriptions for patients participating in CR [5].

In 18 RCTs that compared aerobic exercise-based CR and control groups among patients who underwent PCI or CABG for ACS [93], the aerobic exercise group showed reduced systolic BP (95\% CI, -6.67 to -0.91 ), increased high-density lipoprotein (HDL; 95\% CI, 1.24-6.43), and reduced low-density lipoprotein (LDL; 95\% CI, -10.35 to -0.73) compared to the control group. Furthermore, peak
$\mathrm{VO}_{2}$ (95\% CI, 2.41-4.53) and LVEF (95\% CI, 0.26-4.93) were improved when aerobic exercise was performed. However, there were no significant differences in diastolic BP, total cholesterol, and triglycerides between the two groups. Regarding the effects of aerobic exercise according to duration, exercise for 8 to 12 weeks led to lower systolic $\mathrm{BP}$ at rest and significantly higher peak $\mathrm{VO}_{2}$ compared to the conventional treatment group, and exercise for more than 12 weeks led to positive effects on HDL, $\mathrm{LDL}$, triglycerides, peak $\mathrm{VO}_{2}$, and LVEF. Regarding the amount of exercise per week, 60-90 minutes of exercise per week had positive effects on systolic BP, HDL, triglycerides, peak $\mathrm{VO}_{2}$, and LVEF, and there were no additional effects, even when exercise was performed more than 90 minutes per week (LOE of 1-).

In a systematic literature review comparing the effects of CR with high-intensity interval training (HIT) and that with moderate-intensity continuous training (MCT) [94], HIT led to greater changes of peak $\mathrm{VO}_{2}$ than did MCT (mean difference $[\mathrm{MD}]=1.78$; 95\% CI, 0.45-3.11), while MCT led to lower resting HRs ( $\mathrm{MD}=-1.80 / \mathrm{min}$; $95 \% \mathrm{CI}, 0.71-2.89)$ and body weight ( $\mathrm{MD}=-0.48 \mathrm{~kg} ; 95 \%$ CI, 0.15-0.81) than did HIT. There were no differences in changes to blood glucose, triglycerides, and HDL after exercise between the two exercise groups (LOE of 1+). A Korean study also reported that HIT (1-2 times a week for 9-10 weeks) led to a greater increase in peak $\mathrm{VO}_{2}$, and a 6-minute walking distance led to a greater reduction in perceived depression and fatigue in patients with low to moderate-risk stratification for exercise-related cardiovascular adverse events [95,96].

\section{RECOMMENDATION}

- CR exercise programs should include aerobic exercise (SOR of strong / LOE of $1++$ )

- High-intensity interval training may obtain better results than aerobic exercise (SOR of conditional / LOE of $1+$ )

\section{Resistance exercise program}

CR programs encompass the management of various risk factors that increase the risk of CVD. In particular, interventions for physical activities are a key component of CR [90]. Among different types of physical activities, we presented evidence of resistance training for $\mathrm{CR}$, and reviewed the different effects of CR according to types of 
resistance exercise.

Scotland recommends that aerobic and resistance exercises be considered part of the exercise prescriptions for patients attending CR (SIGN 2017; SOR of conditional / LOE of 1++) [5]. US guidelines are that it is reasonable for a clinician to recommend complementary resistance training at least 2 days per week [5] (AHA 2011; SOR of IIa / LOE of C) [7]. Canada in turn comments that patients in CR may safely derive fitness benefits from a supervised program of resistance training, and resistance training should be offered to all eligible CR participants (CACR 2009) [4].

The exercise component of CR lowers cardiovascular mortality and readmission rates while increasing patients' QOL [91]. The methods, frequencies, durations, and intensities of exercise vary widely across studies, however, it has been reported that CR is effective, regardless of the specific frequency, duration, intensity, and place of exercise (LOE of 1++) [18,19,92].

The SIGN 2017 recommends the inclusion of resistance training in exercise prescriptions for CR [5], and the AHA 2011 reports that resistance exercise improves muscle strength, endurance, fitness, independence, and QOL, regardless of the presence of CVD [7]. One limitation of these studies on the reported effects of resistance training, however, was that they were generally small-scale studies involving low-risk groups for exercise-related adverse cardiovascular events. Thus, the AHA (2011) presents the absolute and relative contraindications of resistance exercise [97] (Table 6).

In a systematic review analyzing the effects of progressive resistance exercise in CR programs, both progressive resistance exercise and aerobic exercise led to increased peak $\mathrm{VO}_{2}$ and fitness, with no significant differences between the two types of exercise. Although the improvement of peak $\mathrm{VO}_{2}$ did not differ between the aerobic exercise plus resistance exercise group and the aerobic exercise only group, the aerobic exercise plus resistance exercise group had greater improvements in maximal exercise capacity. When progressive resistance exercise was performed, there was a significant increase in upper and lower limb muscle strength compared to the control group. When both resistance and aerobic exercises were performed, there was a significant improvement in lower limb muscle strength compared to aerobic exercise alone. Regarding types of exercise, the combined exercise group showed significant improvements when isotonic exercise was performed, but the combined exercise group and the aerobic exercise group did not significantly differ in muscle strength improvements when isokinetic and isometric exercises were used. For lower limb muscles, combining isotonic resistance with aerobic exercises led to significantly better improvements in lower limb muscle strength compared to the effects of using only aerobic

Table 6. Absolute and relative contraindications to resistance training

\begin{tabular}{ll}
\hline & \multicolumn{1}{c}{ Contraindications } \\
\hline Absolute & Unstable coronary heart disease \\
& Decompensated heart failure \\
& Uncontrolled arrhythmias \\
& Severe pulmonary hypertension (mean pulmonary arterial pressure $>55 \mathrm{~mm} \mathrm{Hg})$ \\
& Severe and symptomatic aortic stenosis \\
& Acute myocarditis, endocarditis, or pericarditis \\
& Uncontrolled hypertension $(>180 / 110 \mathrm{mmHg})$ \\
& Aortic dissection \\
& Marfan syndrome \\
& High-intensity resistance training (80\%-100\% of $1 \mathrm{RM})$ in patients with active \\
proliferative retinopathy or moderate or worse non-proliferative diabetic retinopathy \\
Relative (should consult & Major risk factors for coronary heart disease \\
a physician before & Diabetes at any age \\
participation) & Uncontrolled hypertension ( $>160 / 100 \mathrm{mmHg})$ \\
& Low functional capacity (<4 metabolic equivalents of task) \\
& Musculoskeletal limitations \\
& Individuals with implanted pacemakers or defibrillators \\
\hline
\end{tabular}

$\mathrm{RM}$, repetition maximum. 
exercise (LOE of 1-) [98].

In a systematic review of three clinical trials that compared the effects of eccentric and concentric exercises in patients with acute MI, there were no significant differences in peak $\mathrm{VO}_{2}$ and 6 -minute walk test results between the two groups (LOE of 1-) [99].

In a systematic literature review of studies analyzing the effects of CR according to the intensity of resistance exercise, the amount of $\mathrm{VO}_{2 \max }$ increase was greater when lowmoderate-intensity resistance exercise $(<70 \% 1 \mathrm{RM}$ or $\geq 12$ repetition/set) and aerobic exercise were combined, than when aerobic exercise was performed alone. When performed for less than 12 weeks, combined exercise led to a greater increase in $\mathrm{VO}_{2 \max }$ than did aerobic exercise alone, but when performed for more than 12 weeks, studies could not find significant differences in the increases in $\mathrm{VO}_{2 \max }$ between the two groups. One study that examined high-intensity exercise ( $\geq 70 \% 1 \mathrm{RM}$ or $<12$ repetition/set) did not find differences between the two exercise methods. Combined exercise led to superior enhancements in muscle strength compared to those with aerobic exercise, regardless of the intensity and duration of exercise (LOE of $1+)$ [100].

\section{RECOMMENDATION}

- CR programs should include resistance (strengthening) exercises (SOR of conditional / LOE of $1++$ )

Safety issues of exercise training in cardiac rehabilitation The safety of patients who have experienced ACS must be ensured during CR. To this end, it is universal practice to assess patients' cardiac states after ACS, stratify risk for exercise-related cardiovascular adverse events through cardiopulmonary exercise testing, and have patients perform ECG-monitored exercise at the hospital for a certain period early after onset.

With regard to safety issues related to CR and exercise, US recommendations are that all patients receive a risk assessment with a physical activity history and/or an exercise test to guide prognoses and prescriptions, and the clinician should counsel patients to report and be evaluated for symptoms related to exercise (AHA 2011; SOR of I / LOE of B, C) [7]. Canada advises that risk stratification is the key to safe and effective exercise prescriptions, and all patients entering CR programs must have a medical assessment and undergo determination of their cardio- metabolic fitness prior to the initiation of therapy. A directly supervised GXT is recommended as part of the initial CR assessment prior to the initiation of therapy, and the provision of continuous EKG telemetry should be at the discretion of the CR program medical director. Patients in CR are encouraged to wear a heart rate monitor during physical activity and exercise, and follow the target heart rates prescribed for them (CACR 2009) [4].

Findings such as $\mathrm{VO}_{2 \max }$, duration of exercise testing, presence of angina symptoms, and ECG abnormalities from cardiopulmonary exercise testing are helpful for predicting long-term outcomes such as mortality for patients who underwent PCI or CABG for ACS [101-105]. Furthermore, demographic factors and CVD history, such as sex, age, BMI, smoking, systolic BP, total cholesterol, HDL, hypertension, diabetes, and drug history are known to be predictors of CVD recurrence and mortality $[4,106]$.

Therefore, risk stratification for exercise-related cardiovascular adverse events based on history and cardiopulmonary exercise testing is important for patients who undergo PCI or CABG [7]. Risk stratification is critical for ensuring the effectiveness and safety of exercise prescriptions, and therefore, cardiopulmonary exercise testing is recommended prior to beginning an initial CR exercise program [4]. The incidence of heart attacks when conducting exercises such as jogging, swimming, playing tennis, and engaging in cross country skiing is reported to be higher in the heart disease population than in the population without heart disease, with one case per $12,000-121,955$ hours, and one case per $375,000-888,000$ hours, respectively [107]. However, appropriate exercise programs significantly lower the disease-related mortality of patients with heart disease, so recommending aggressive CR exercise training to these patients is a reasonable decision in which the benefits far outweigh the harms. The type and intensity of exercise and monitoring during exercise are known to have a serious impact on exercise-related deaths, and continuous ECG monitoring markedly lowers the incidence of heart attacks during exercise [107].

According to one Korean report, there were 17 cases of angina symptoms without ECG anomalies, 31 cases of ECG anomalies without angina symptoms, 10 cases of ECG anomalies with angina symptoms, and 10 cases of temporary blood pressure instability during 13,934 hours 
of CR by 975 patients over a 10-year period, but during this time there was not a single major adverse cardiac event, such as a death or AMI [108].

\section{RECOMMENDATION}

- Risk assessment and appropriate monitoring based on the risk assessment results should be performed to ensure patient safety during CR exercise programs (SOR of strong / LOE of 2++)

\section{Home-based cardiac rehabilitation}

Home-based CR does not refer to an exercise performed alone by the patient at home; it is a form of exercise performed at home after medical professionals have assessed the patient's cardiac functions at the hospital through exercise testing, and the patient has been given an exercise prescription and education about risk factor management. Effective home-based CR is based on continuous bidirectional communication with the medical team, and continuous follow-up assessments. Owing to recent advances in information and communication technologies (ICTs) and medical technologies, studies are continuing to examine new approaches to homebased CR and their effects. Hence, we reviewed relevant evidence and prepared a recommendation tailored to the circumstances in Korea.

Scotland's guidelines state that an exercise component in CR reduces cardiovascular mortality and hospital admissions, and improves health related QOL regardless of the type of CHD, type of CR, or setting. Benefits appear to be independent of a specific frequency, duration, or intensity of exercise, or whether it takes place in a hospital, home, or community setting. Further, in homebased CR programs, BCTs such as social supports and goal setting were shown to be effective in reducing CVD risk factors, with results comparable to those of hospitalor center-based programs. It concluded that there is little difference between home- and center-based CR in terms of the number of program withdrawals (SIGN 2017; LOE of 1++) [5]. US guidelines are that a home-based CR program can be substituted for a supervised, center-based program for low-risk patients (AHA 2011; SOR of I / LOE of A) [7]. Canada advises that CR programs should follow comprehensive program models, both home-based and institution-based, and integrate the core program components presented within its guidelines (CACR 2009) [4].
Previous studies have proposed several methods of home-based CR, including one in which patients perform $\mathrm{CR}$ at home by themselves after undergoing a cardiac function assessment at the hospital and being given an exercise prescription, with periodic visits from a therapist to provide further instructions on CR. Another approach involves teaching patients to exercise using a brochure or phone, and monitoring their adherence [109].

A systematic literature review that analyzed the effects of home-based CR and hospital-based CR found no differences between the two groups in total mortality at 12 months after onset ( $R R=1.19$; 95\% CI, 0.65-2.16), fitness at 12 months after onset $(\mathrm{MD}=-0.13 ; 95 \% \mathrm{CI},-0.28$ to 0.02 ), fitness at 12 to 24 months after onset, quality of life parameters at 24 months, CR completion rates, and total cholesterol, LDL, triglycerides, systolic BP, diastolic BP, and smoking cessation rates at 3 months and 12 months (LOE of 1+) [110,111]. Furthermore, home-based CR and hospital-based CR had equal effects on maximum heart rate and weight loss (LOE of 1-) [111]. Similarly, both CR programs had equal effects on physical and emotional components of QOL as well as on depression (LOE of 1-) [111]. Regarding anxiety, one study reported that homebased CR and hospital-based CR had similar levels of effectiveness [111], and another study reported that homebased CR was slightly more effective (LOE of 1-) [112]. Regarding blood HDL, one study found increased blood HDL after hospital-based CR, [110] while another study found hospital-based CR to be more effective, though the statistical significance of the difference was unclear [111], thus calling for additional analysis (LOE of 1+). Behavioral change techniques such as social supports and goal setting during home-based CR programs are effective in decreasing cardiovascular risk factors, and the levels of effects were similar to those of hospital-based CR programs (LOE of 1++) [113].

A report conducted in Korea suggested that homebased CR programs using a mobile ECG data transmission device (HeartCall) led to improvements in the participants' fitness and QOL [114]. Owing to recent advances in ICTs, researchers in Korea are attempting to study CR equipment based on smart devices and sensors, and additional studies are needed to investigate the clinical effects of home-based CR programs using these technologies $[115,116]$. 


\section{RECOMMENDATION}

- Home-based CR programs may replace hospital-based CR programs for low-risk patients (SOR of conditional / LOE of 1++)

\section{Cardiac rehabilitation for elderly patients}

The need for CR is also growing due to aging and the subsequent rise of coronary syndromes among those aged 65 years and older. In many cases, however, the elderly engage in fewer daily physical activities, and have a low level of participation in CR exercise programs due to other comorbidities. Based on our review of the relevant literature, we developed recommendations for CR for the elderly.

Canada's guidelines state that elderly patients with CAD will benefit from and should participate in CR programs that include both aerobic and resistance training (CACR 2009) [4].

One-year survival rates for acute MIs is known to decline with advancing age [117]. There is also a report that those aged 65 years or older who have had MIs show lower $\mathrm{VO}_{2 \max }$, exercise testing durations, depression indexes, and grip strength compared to those with other heart diseases [118]. According to an article that reviewed various studies observing the effects of CR by age, CR improves aerobic fitness and physical functioning scores equally, and lowers body fat percentages and LDL/HDL ratios in patients 65 years and older similarly to its effects on patients under the age of 65 [119]. Even among the superaged over 75, CR can lead to functional improvements, and for this group it is important to initiate CR aggressively in the acute phase, immediately after being admitted for heart disease, and continuing into hospitalcentered outpatient CR after discharge [120]. Therefore, elderly patients with CAD must also be provided with and be motivated to participate in CR that is comprised of aerobic and resistance exercise [4]. A systematic literature review that analyzed the effects of resistance training on elderly ( $\geq 65$ years) patients and mid-aged patients with CAD also found that resistance training improves upper and lower limb muscle strength, exercise capacity, and mobility equally in both patient groups (LOE of $1++$ ) [121].

Although no Korean study has analyzed the effects of CR in those aged 65 years and older, it has been reported that $\mathrm{CR}$ improves $\mathrm{VO}_{2 \max }$ and anaerobic thresholds in $\mathrm{MI}$ in patients aged 60 years and older [122].

\section{RECOMMENDATION}

- CR programs should also be administered to patients aged 65 years and older (SOR of strong / LOE of 1++)

\section{Education for secondary prevention}

Need for educational components in cardiac rehabilitation

The British Association for Cardiovascular Prevention and Rehabilitation (BACPR) defines CR as the collection of all behaviors aiming to delay or reverse disease progress by rectifying health behaviors and returning to an ideal social life or preserving social functions [123]. Other CR guidelines use similar definitions, and thereby confirm that existing CR programs are oriented to providing a comprehensive program that consists of secondary prevention and improvements in patients' QOL, as opposed to simply focusing on their rehabilitation with reduced motor functions.

Behavioral interventions that focus on directing patients' health behaviors in a desirable direction are needed with regard to various aspects such as physical activities, diet, smoking cessation, body weight management, stress management, and psychological problems, and there is high demand for CR programs that encompass these factors, though they are not adequately addressed in conventional clinical practices. Furthermore, although maintaining various drug therapies such as statins, antiplatelets, and beta-blockers is well supported by evidence and plays an important role, the issue of whether patients are actually adhering to these drug therapies is influenced by diverse factors that involve the patients, medical teams, prescriptions, and healthcare systems. There is evidence suggesting that the ideal state has not yet been reached, and CR programs must also address this shortcoming.

Providing knowledge about a patient's disease, subsequent treatments, the expected effects and needs, precautions and self-care efforts is important for assessing their effects on patient outcomes, but also has ethical implications in terms of ensuring patients' rights to know.

Scotland's guidelines reviewed the evidence for what was at that time called 'comprehensive rehabilitation. This term was used to define the prevalent model of CR, which consisted of two components, exercise and educa- 
tion. They concluded that when helping patients make lifestyle changes, CR programs should place comparable emphasis on each of the lifestyle risk factors (SIGN 2017; SOR of strong) [5]. England's guidelines observed that comprehensive CR programs should include health education and stress management components (NICE 2013; SOR of I) [6]. Japan has recommended that CR programs include patient education sessions (JCS 2012; SOR of I / LOE of A) [8].

In a review of 6 RCTs that compared exercise-only groups and exercise and education groups of patients with CAD, exercise and education about risk factors increased patients' levels of physical activities over 6-12 months, but their effects on smoking and diet were not clear; the strategies employed here included goal setting, problem solving, self-monitoring, and role-modeling (LOE of 1++) [79]. Other results indicated that similar strategies were effective after participating in a CR program (limited to exercise training), and even when not participating in a CR program (LOE of 2-) [124].

In a systematic review of patients with ACS, only one RCT examined the effects of an intervention aimed to increase self-efficacy. This trial reported that there were no significant differences in the levels of physical activities between the intervention and conventional treatment groups at 6 months (LOE of 1+) [77]. However, when the results of all qualitative studies on patients with heart failure were combined, patients' responses to their bodily changes and the extent of encouragement to exercise received from friends and family members were associated with their levels of physical activity, suggesting that enhancing self-efficacy and helping patients have positive expectations of their results may be important factors for inducing behavioral change (LOE of 1+) [125]. In a systematic literature review of studies that analyzed the effects of home-based CR programs, 10 out of 11 RCTs found that behavior change techniques were useful in lowering controllable CVD risk factors, and the techniques used included social support, goal setting, and self-monitoring (LOE of 1++) [113].

When articles published after the publication of existing CPGs were searched, of 22 clinical trials, one study found that the effects of educational interventions were appropriate to answering the key question (LOE of 1+) [126]. Most clinical trials included in this study had low risk of bias, and educational interventions varied widely, from 40-minute interviews with 15-minute phone follow-ups, to 4 -week programs with 11 months of follow-up sessions. Most studies reported only all-cause mortality, and few studies compared mortality rates by cause of death. There were significant differences in CVD mortality rates according to educational levels, suggesting that educational interventions may be effective, but there were only two relevant studies, and the level of evidence is low. The two groups did not significantly differ in all-cause mortality, MI, revascularization rates, and admission rates, and the educational intervention group tended to show higher QOL indices, though they were not consistently high across all indices.

Because the effects of educational interventions are more indirect-as in altering patient behaviors-as opposed to influencing the outcomes of CVD directly, they may vary greatly according to the patient population's characteristics, the method/intensity/duration of an intervention, and whether patients accepted the interventions. It is difficult to conclude that simply summing the results alone could describe the effects of educational interventions in their entirety. Furthermore, there are cases in which the control group may alter its behaviors independently, thereby diluting the effects of interventions. When interpreting the results, therefore, the effects of educational interventions are much more difficult to substantiate than those of drug therapies or procedures. Given this background, we strongly recommend that patient education be included in CR programs, despite the low evidence level supporting the effects of educational interventions, and the low effects these interventions are documented to have in the literature.

\section{RECOMMENDATION}

- CR programs should include patient education (SOR of strong / LOE of $1++$ )

\section{Contents of patient educational programs}

Existing CPGs for CR make recommendations about the information that should be provided to patients, that is, the contents to be included in patient education. Supporting evidence is thin, however, and there is a lack of adequate evidence to support each specific item.

With regard to the content of patient education materials, one study reported that many patients voluntarily stopped taking prescribed medicines, and even those 
who continued to take them often took them irregularly, with adherence rates merely reaching an average of $50 \%$ in the long-term [127]. Several drugs such as statins and antiplatelets provide firm evidence of improved outcomes from ACS. Helping patients adhere to these drug therapies is crucial for secondary prevention, and CR programs may serve as an opportunity to achieve this end.

Scotland's guidelines recommend discussing the impacts of a cardiac event/coronary heart disease on emotional well-being throughout $\mathrm{CR}$, advising patients about the purpose and use of secondary prevention medication and encourage concordance, while linking patients to other information sources, peer support, and support groups. Prior to hospital discharge following a cardiac event, verbal and written information on diagnosis, chest pain management-including how to use the glyceryl trinitrate (GTN) spray-advice on driving, returning to work, and appropriate daily activities should be provided, including information such as booklets from the British Heart Foundation or Chest, Heart \& Stroke Scotland. Future treatments, interventions, and appointments should be discussed, along with the purpose of CR, and a contact number for the CR unit should be provided. The benefits of smoking cessation should be discussed if appropriate. At the CR assessment, information appropriate to the needs and choices of the patient with regard to activities, exercise, smoking cessation, weight management, diet, and common emotional adjustment reactions to ill health should be provided, and the patient should be advised of the benefits of continuing to exercise long term, and assured that it can be undertaken safely and effectively in any setting. The patient should also be given a contact number for ongoing person-centered advice (SIGN 2017) $[5,8]$.

Japan encourages (1) teaching the patient how to manage chest pain, and providing contact information in the event unmanageable chest pain occurs; (2) instructing the patient on the use of nitroglycerin sublingual tablets or sprays; (3) encouraging the patient and his/her family members to learn how to perform cardiopulmonary resuscitation; (4) explaining the patient's coronary risk factors; (5) motivating the patient to participate in CR and improving their lifestyle; and (6) encouraging the patient to stop smoking and maintain a tobacco-free lifestyle (JCS 2012) [8].
The authors did not review the level of evidence for specific educational content, but did review evidence concerning adherence to drug therapies by reviewing the published literature. SIGN 2017 found that most studies with high levels of evidence and high effects used multifaceted and complex interventions, including intensive education and counseling by various medical and nonmedical experts, continuous treatment support, as well as family and coworker support, and given that the heterogeneity across studies was high, concluded that it was difficult to present specific recommendations (LOE of $1++)$ [127].

Other evidence showed that early interventions by a nurse and those in which the physician in charge was provided with information about a patient's adherence to drug therapy were effective albeit to a small extent [128]. Multiple recent studies, as well as new studies, are continuing to investigate the effectiveness of mobile health using cell phone messages or smartphone applications, and systematic reviews of completed studies found that these techniques are effective to some degree (LOE of 1-) [129-132]. Even so, there is significant heterogeneity regarding the methods involved, so it is difficult to make specific recommendations about the interventions that should be used to improve adherence.

Medication adherence remains a crucial issue that cannot be neglected, necessitating that CR teams confirm and evaluate whether patients are adhering to their prescribed medications. CR programs have great potential to provide a tool for checking and improving patients' adherence, but additional studies are needed to confirm which interventions should to be integrated in CR programs to effect this outcome.

\section{RECOMMENDATION}

- The recommendations in SIGN 2017 should be largely accepted regarding educational contents (SOR of GPP / LOE of 2+) (Table 7)

\section{Education for smoking cessation}

The importance of smoking cessation by patients with ACS and the general population is undoubtable. However, there is currently inadequate evidence documenting the specific benefits of smoking cessation interventions in CR programs, and existing structures and intensities of such interventions are inadequate. These interventions 
Table 7. Education contents for cardiac rehabilitation in SIGN 2017 [5]

\begin{tabular}{ll}
\hline & \multicolumn{1}{c}{ Education contents } \\
\hline $\begin{array}{l}\text { Throughout entire } \\
\text { CR program }\end{array}$ & $\begin{array}{l}\text { - Education about the effects of cardiovascular events/coronary artery disease, such as } \\
\text { myocardial infarction, on psychological/emotional state }\end{array}$ \\
& - To promote secondary prevention, explain the purpose and need for the prescribed \\
& medications and encourage patients to adhere to prescription-guide patients to additionally \\
& obtain appropriate information
\end{tabular}

SIGN, Scottish Intercollegiate Guidelines Network; CR, cardiac rehabilitation.

require additional staff resources, and because the availability of these resources varies across hospitals in Korea, each organization's circumstances must be considered when including this intervention. In general, an adequate intensity of intervention is most likely to determine the effects of a behavioral therapy, so we recommend an appropriate follow-up period. Nonetheless, programs should be designed on the basis of the human resources available, the extent to which these resources could be dedicated to CR programs or educational efforts that include smoking cessation, and the expected outcomes.

Scotland's guidelines recommend that CR patients who smoke should be offered smoking cessation interventions that extend over more than 4 weeks. These interventions should include telephone contact, behavioral support, and self-help materials (SIGN 2017; SOR of strong / LOE of 1++) [5]. US recommendations are that tobacco users should be advised to stop smoking at every visit, patients should receive counseling and help developing a plan to stop smoking that may include pharmacotherapy and/ or referral to a smoking cessation program (AHA 2011; SOR of I / LOE of A), and all patients should be advised at every office visit to avoid exposure to environmental tobacco smoke at work, home, and in public places (LOE of B) [7].

A systematic review of published studies found that psychosocial interventions were effective in achieving smoking cessation [133]. It is known that behavioral therapies, phone support, and self-help have similar effects, but most programs combined two or more approaches. The effects are closely associated with the duration of interventions, but programs that lasted for more than a month after initial contact (RR=1.28; 95\% CI, 1.17-1.40), were more effective than those that lasted for less than a month (RR=1.01; 95\% CI, 0.91-1.12) (LOE of 1++). Phone support interventions were also found to be very effective in promoting smoking cessation $(\mathrm{RR}=1.32$; $95 \% \mathrm{CI}, 1.07$ 1.62). The current review includes both $C R$ programs that included phone support interventions and those that did not (LOE of 1-) [134].

In a systematic review of studies that assessed interventions begun during patients' hospital stays and continued during outpatient visits, only those that extended over more than 1 month were effective. Diverse CVD patients were studied, and some were offered nicotine replacement therapies (LOE of 1++) [135]. A review of studies based on interventions delivered by nurses found them to be effective. These interventions included counseling sessions where patients' were encouraged to stop smoking, benefits and factors that hindered smoking cessation were reviewed along with coping strategies, and followup visits were incorporated. This review noted that five 
studies found significant effects associated with CR programs that had an educational component ( $\mathrm{RR}=1.35 ; 95 \%$ CI, 1.14-1.59). Interventions led by a nurse responsible for CR and health education components delivered over several sessions were more effective than interventions led by a nurse who was also involved in other tasks (LOE of 1+) [136]. Combining therapy with face-to-face or phone behavioral interventions increased the success rates of patients undergoing nicotine replacement therapy by $10 \%-25 \%$ (LOE of $1++$ ) [137].

Regarding evidence published after the publication of CPGs, Franck et al. [138] conducted a systematic review of seven studies with a patient population similar to that in this study. It included three intervention studies focused on counseling, and four studies that combined drug therapy with counseling. Of the studies that used drug therapy, only the one that used varenicline found a significant increase in the smoking abstinence rate, and three behavioral intervention studies that significantly improved the rate of smoking abstinence at 6 months and 12 months were assessed as having problems with generalizability. All-cause mortality was not reduced, and there were no significant differences in the recurrence of MI, revascularization, and readmission. Two studies documented a significant decrease in cardiovascular events. Though there was a tendency to improve QOL, this improvement was not consistent across all indices. If limited to the additional evidence, smoking cessation interventions do seem useful for ACS, but there is not a high level of evidence supporting this supposition (LOE of 1-).

\section{RECOMMENDATION}

- Smoking cessation interventions should be provided for patients who smoke, and continuous interventions of more than 4 weeks should be considered (SOR of strong / LOE of 1++)

Diet program for patients with cardiovascular disease (proposal by the Korean Society of Clinical Nutrition)

The diet program for CR patients proposed by the Korean Society of Clinical Nutrition aims to help these individuals maintain a healthy weight, appropriate blood lipid levels, and blood pressure. Since diet programs must consider a country's general meal patterns and cultural backgrounds, it is inappropriate to follow existing Ameri- can or European recommendations, and it is unrealistic to review the levels of evidence for each specific item, as is the case with educational contents. We therefore present GPPs in collaboration the Korean Society of Clinical Nutrition.

These guidelines were developed on the basis of recently revised major dietary guidelines related to CVD prevention and rehabilitation in Korea and abroad [46,9,139-142]. The Mediterranean diet, one of the most widely accepted healthy dietary patterns, has been reported to lower CVD risks in 5-year meta-analyses of RCTs and cohort studies [143,144], and has also been reported to significantly lower morbidity and mortality in CVD secondary prevention intervention studies that include comprehensive nutritional interventions [145-147]. More systematic studies are needed, however, since there are few relevant Korean data that can be used to propose levels of evidence and assess the strengths of recommendations for each item in this dietary guideline.

\section{RECOMMENDATION}

- Diet programs should be designed with reference to the following guidelines (SOR of GPP / LOE of 2+) (Table 8)

\section{Recommendations for food supplements}

As is true among patients with other acute or chronic diseases, many patients with CVD take food supplements and are interested in them. Often they also consult their physicians about them, and physicians must respond appropriately, thereby necessitating a review of evidence regarding the use of food supplements. Patients are interested in or take diverse types of food supplements, but it is impossible to address all of them in detail. For this reason, we developed a recommendation for the food supplements most commonly encountered in practice: omega-3 fatty acids, polycosanol, and antioxidants. Although the available evidence is inadequate, by carefully generalizing these recommendations, it was possible to give levels of recommendations for food supplements (LOE of 4).

\section{Omega-3 fatty acids}

England advises physicians not to offer or advise people to use the following to prevent another MI: omega-3 fatty acid capsules and/or foods supplemented with omega-3 
Table 8. Diet program for cardiac rehabilitation program

\begin{tabular}{|c|c|c|}
\hline Item & Contents & Notes \\
\hline Diet pattern & $\begin{array}{l}\text { Diversify food groups within the appropriate } \\
\text { total energy consumption to maintain a } \\
\text { healthy weight, including whole grains, } \\
\text { vegetables, fruits, fish (blue-backed fish), } \\
\text { poultry, beans, and nuts. }\end{array}$ & $\begin{array}{l}\text { Major foreign guidelines prioritize a guideline for } \\
\text { overall diet patterns. Korean guidelines do not } \\
\text { provide instructions about diet patterns, but it would } \\
\text { be desirable to include one, owing to the nature of } \\
\text { the diet culture. However, considering Koreans' food } \\
\text { culture, we did not mention dairies (low fat). }\end{array}$ \\
\hline Fat $[141]$ & $\begin{array}{l}\text { Limit total fat intake to } 30 \% \text { of total energy } \\
\text { intake. Limit saturated fat intake to } 7 \% \text { of } \\
\text { total energy intake. Replace saturated fats } \\
\text { with monounsaturated fatty acid (MUFA) } \\
\text { and polyunsaturated fatty acid (PUFA), and } \\
\text { limit omega- } 6 \text { PUFA to } 10 \% \text { of total energy } \\
\text { intake. Limit trans-fat intake to } 1 \% \text { of total } \\
\text { energy intake. }\end{array}$ & $\begin{array}{l}\text { Generally, accept the recommendations of the Korean } \\
\text { Society of Lipid and Atherosclerosis (KSLA) guideline } \\
\text { (However, to increase the emphasis on MUFA, we } \\
\text { revised the guideline to separately mention MUFA } \\
\text { and PUFA). }\end{array}$ \\
\hline $\begin{array}{l}\text { Cholesterol } \\
{[141,175]}\end{array}$ & Limit daily cholesterol intake to 300 mg. & $\begin{array}{l}\text { Recently, multiple foreign guidelines tend to delete a } \\
\text { guideline for cholesterol. However, we included this, } \\
\text { as some foreign guidelines still limit daily cholesterol } \\
\text { intake to } 200-300 \mathrm{mg} \text {, and the Korean nutrient } \\
\text { standard and KSLA guideline limits daily cholesterol } \\
\text { intake to } 300 \mathrm{mg} \text {. }\end{array}$ \\
\hline Salt $[176,177]$ & $\begin{array}{l}\text { Limit daily salt intake to } 5 \mathrm{~g} \text { (daily sodium } \\
\text { intake to } 2 \mathrm{~g} \text { ). }\end{array}$ & $\begin{array}{l}\text { Daily salt intake ranges from } 4-6 \mathrm{~g} \text { in foreign } \\
\text { guidelines. To remain consistent with the Korean } \\
\text { nutrient standard, we set daily salt intake to } 5 \mathrm{~g} \text {. }\end{array}$ \\
\hline $\begin{array}{l}\text { Fibers } \\
{[178,179]}\end{array}$ & $\begin{array}{l}\text { Eat enough whole grains and vegetables to } \\
\text { keep daily fiber intake above } 25 \mathrm{~g} \text {. }\end{array}$ & $\begin{array}{l}\text { European guidelines set daily fiber intake to } 30-45 \mathrm{~g} \text {, } \\
\text { but Canadian, American, and Korean guidelines set } \\
\text { the cutoff to } 25 \mathrm{~g} \text {. }\end{array}$ \\
\hline Sugars & $\begin{array}{l}\text { Limit added sugar (sugars added during } \\
\text { cooking or processing) intake to } 10 \% \text { of } \\
\text { total energy intake. }\end{array}$ & $\begin{array}{l}\text { Most foreign major guidelines include a phrase about } \\
\text { limiting sugar-sweetened beverages in their diet } \\
\text { pattern guideline. Because sugar intake is on the } \\
\text { rise recently, it is valid to include sugar intake in the } \\
\text { guideline. As with the added sugar standard in the } \\
\text { Korean nutrient standard, added sugars are limited } \\
\text { to } 10 \% \text { of total energy intake. }\end{array}$ \\
\hline Alcohol [142] & It is desirable to avoid drinking. & $\begin{array}{l}\text { In unavoidable cases, alcohol should be limited to } \\
2 \text { shots a day for men }(20 \mathrm{mg}) \text { and } 1 \text { shot a day for } \\
\text { women }(10 \mathrm{mg}) \text {. Alcohol intake should be minimized } \\
\text { to control blood pressure and body weight. }\end{array}$ \\
\hline
\end{tabular}

fatty acid. There is, however, no evidence of harm if people choose to take these capsules or foods. It further advises people not to take supplements containing betacarotene, and does not recommend antioxidant supplements (vitamins $\mathrm{E}$ and/or C) or folic acid to reduce cardiovascular risk (NICE 2013) [6].

Patients who had MIs and take omega- 3 supplements or increase the $\alpha$-Linolenic acid (ALA) content in their diet for secondary prevention will not experience negative consequences. Further, omega-3 or ALA does not have a harmful effect on lipid metabolism, and may also reduce triglycerides [148]. However, there is no clear evidence suggesting that they influence cardiovascular events or all-cause mortality (LOE of 1++) [149].

There have been reports of relatively mild adverse events, such as diarrhea, nausea, delayed hemostasis, and allergies after taking omega-3 as food supplements, but omega-3 lowers triglycerides and increases HDLcholesterol. Several clinical trials and meta-analyses, however, found little evidence suggesting that taking 
omega-3 lowers all-cause mortality, cardiovascular events, or cardiovascular mortality, the incidence of CAD, cerebrovascular disease, or arrhythmia [149-152]. An ALA-rich diet did not lower all-cause mortality or cardiovascular mortality, and its effects on the incidence of stroke were not clear. It did, however, slightly reduce deaths from CAD and the incidence of arrhythmia [149].

\section{Polycosanol}

In a study that assessed the effects of policosanol in patients with hyperlipidemia, polycosanol lowered total cholesterol and LDL-cholesterol and increased HDLcholesterol with no significant effects on triglycerides or body weight [153]. Patients with a history of CAD must take high-dose statins for secondary prevention purposes, however, in cases where side effects included poor drug adherence and necessitated the use of lowdose statins, combining low-dose statins with Armolipid Plus (red yeast rice, policosanol, berberine, folic acid, coenzyme Q10, astaxanthin complex) led to a greater reduction of LDL-cholesterol and higher achievement of targeted LDL-cholesterol rates without differences in adverse events (LOE of 1-) [154].

Another study that assessed the safety and effects of adding polycosanol for patients receiving antiplatelets after drug eluting stent insertions found that adding polycosanol did not significantly elevate cardiovascular events, deaths, or bleeding tendencies (LOE of 1-) [155]. However, findings on the association of polycosanol and improvements in dyslipidemia are inconsistent [156], and there have been no clinical trials on the long-term clinical outcomes or secondary prevention outcomes related to polycosanol intake in patients with CAD.

\section{Antioxidants}

Vitamin E, beta-carotene, and vitamin $\mathrm{C}$ are antioxidants widely used as food supplements. Vitamin C has been found to delay the progression of early atherosclerosis, with no benefits for atherosclerosis in later stages in vitro. A meta-analysis of the effects of these antioxidants on major cardiovascular events found that there were no significant differences between these antioxidants and placebos in major cardiovascular events, that is, MI, stroke, all-cause mortality, heart disease-related mortality, revascularization rates, CAD, and heart failure [157]. Further, antioxidants did not prevent cardiovascular events in women (LOE of $1++)$ [158,159].

It has been reported that vitamin $\mathrm{E}$ lowers the incidence of MI and stroke in patients aged 65 years or older [160], but this was only observed in a RCT that involved supplements manufactured by a pharmaceutical company, and there is a lack of evidence suggesting that it has preventive effects against CVD (LOE of 1++) [157,161]. Given published findings and the results of the analyses conducted in developing these CPGs, there is not sufficient evidence to conclude definitively that omega-3, polycosanol, and antioxidants have benefits for the secondary prevention of CAD.

It would clearly be harmful if patients overestimated the effects of these food supplements and deluded themselves into thinking they were undergoing adequate treatment, and so paid less attention to adhering to other established measures for secondary prevention, that is, taking prescribed medicines, exercising, and giving up smoking. Therefore, healthcare professionals must intervene aggressively in this matter (LOE of 4).

\section{RECOMMENDATION}

- Food supplements are not recommended for secondary prevention of CAD (SOR of conditional)

\section{ICT-based modality for patient education}

ICTs (e.g., phones, cell phones/smartphones, mobile apps, tablet computers, the Internet, biosensors) are now widely used in healthcare. The World Health Organization defines medical and public health practice using mobile devices (e.g., cell phones, patient monitoring devices, personal digital devices, other wireless devices) as mobile health (mHealth) [162], though its definition is still vague, and it is often confused with electronic health (eHealth) and telehealth. This technology can, however, help overcome factors that limit participation in CR, such as distance, traveling, scheduling, access to caregivers, and costs. These technologies can increase access to home-based CR, enable two-way communications with experts and monitoring by experts, and is also helpful for hospital-based programs. In particular, mHealth is very useful for managing chronic diseases such as CVD, by increasing a patient's knowledge and awareness of their disease, and providing appropriate education to induce and maintain good self-care.

As of 2015, Internet usage rates and smartphone pen- 
etration rates among adults in advanced countries worldwide were $87 \%$ and $68 \%$, respectively. At $94 \%$ and $88 \%$, respectively, these rates are highest in South Korea. Although there is a great gap in developing countries, which have rates of about $54 \%$ and $37 \%$ respectively, rates in these countries are rising sharply [163]. As of $2017,50 \%$ of global Internet traffic was on mobile devices (smartphones or tablet computers) [164], and mHeatlh apps were estimated to have been downloaded 3.7 billion times [165]. In other words, the Internet and mobile digital devices based on ICTs that are already a routine part of our daily lives are widely used for health-related purposes.

Scotland's guidelines are that technology-based interventions should be considered for patients participating in $\mathrm{CR}$, and psychoeducation (goal setting, self-monitoring) should be considered for patients in CR to facilitate adherence to engaging in physical activities (SIGN 2017; SOR of conditional / LOE of 1++) [5].

The results of ICT-based interventions intended to promote exercise are not consistent $[111,166]$. An Australian patient-centered program that consisted of a 1-hour initial consultation followed by 3 months of repeated phone follow-ups significantly reduced total cholesterol (mean $154.7 \pm 3.9$ vs. $181.8 \pm 3.9 \mathrm{mg} / \mathrm{dL} ; \mathrm{p}<0.001$ ), systolic BP (mean $131.6 \pm 1.8$ vs. $143.9 \pm 2.3 \mathrm{mmHg}$; $<0.001$ ), and BMI (mean $28.9 \pm 0.7$ vs. $31.2 \pm 0.7 \mathrm{~kg} / \mathrm{m}^{2} ; \mathrm{p}=0.025$ ) [166]. In another multicenter RCT in Australia, goal setting with 6 months of phone follow-ups led to a greater reduction in total cholesterol (MD=21 mg/dL and 95\% CI, 16-25 $\mathrm{mg} / \mathrm{dL}$ vs. $\mathrm{MD}=7 \mathrm{mg} / \mathrm{dL}$ and $95 \% \mathrm{CI}, 3-11 \mathrm{mg} / \mathrm{dL}$ ), body weight, BMI, dietary fat intake, saturated fat intake, and anxiety [166]. These interventions, which had efficacies similar to those of traditional CR, may serve as alternatives for patients who cannot participate in hospitalbased CR, or those who simply prefer this method (LOE of 2+) $[111,166]$. In a RCT that used exercise prescriptions and behavior support over the Internet and text messages (4-6 per week for 24 weeks) for patients with ischemic heart disease (mean age, 60 years), this intervention did not improve exercise capacity $\left(\mathrm{VO}_{2 \max }\right)$, but significantly increased leisure activities ( $\mathrm{MD}=110.2 \mathrm{~min} /$ week; 95\% $\mathrm{CI},-0.8$ to $221.3 ; \mathrm{p}=0.05)$ and walking time $(\mathrm{MD}=151.4$ $\mathrm{min} /$ week; 95\% CI, 27.6-275.2; $\mathrm{p}=0.02$ ). In addition, patients' self-efficacy and QOL were significantly improved (LOE of 1++) [167]. The use of digital pedometers in a small-scale RCT significantly increased the total number of physical activities, walking time, and walking frequency of patients undergoing CR (LOE of 1+) [168].

Combining physical activities with video games increased involvement in physical activities and improved QOL, well-being, and symptoms of depression in patients with stroke or heart failure aged 50 years or older. These attempts were evaluated to be safe and fun (LOE of 2+) [169]. Internet-based interventions, such as websites that provided individual instructions, also improved the number of steps and exercise capacity of patients in distant locations, or those who had decided not to participate in hospital-based CR (LOE of 1++) [71,170,171]. In a home-based RCT that provided a web-based rehabilitation program for patients with angina, at the 6-week follow-up the intervention group showed an average increase of 497 steps daily, while the control group showed an average decrease of 861 steps daily (LOE of 1+) [170]. Patients who were provided with 6 months of online personal instruction called CardioFit, an Internet-based system developed to promote physical activities in patients with CAD, increased the amount of physical activities they engaged in and their QOL at 6 months and 12 months (LOE of 1++) [172]. In addition, the intervention group, which used an Internet-based virtual CR program for 4 months, showed a significant increase in maximum treadmill duration (45.7 seconds; 95\% CI, 1.04-90.48) at 16 months, compared to that of the control group. The intervention group also had decreased total cholesterol, LDL-cholesterol, and dietary saturated fat intake (LOE of 1++) [171]. Home-based multilateral CR models using ICT were associated with improvements in CVD risk factors comparable to those of traditional hospital-based CR programs [166]. Thus, these CR models may be integrated into traditional treatment models, and increase the number of options available to patients (LOE of 2+).

A recent study reviewed and meta-analyzed 10 clinical trials of 607 patients in 5 countries about the utility, acceptance, and usefulness of mobile apps for self-care of CVD and management of risk factors [173]. The mobile app group showed improvements in readmission rates, disease knowledge, psychosocial well-being, BMI, waist circumference, cholesterol and fitness, physical activities, drug adherence, and smoking cessation rates [173]. However, many of these studies were small-scale, had a short study period, had no blinding due to the nature of 
the intervention, and used biased data, thereby necessitating additional studies to address these shortcomings. Nevertheless, these studies confirmed the potential mobile apps had for inducing behavioral changes and ameliorating CVD risk factors. These findings allude to the usefulness of mHealth as a healthcare delivery system that improves access to CR for patients who have difficulty participating in traditional hospital-based CR (LOE of 1-).

Maintaining medication adherence is critical for the secondary prevention of CVD, and is associated with readmission, disease onset, mortality, and increased medical costs [174]. Hence, behavioral interventions such as education and counseling intended to enhance medication adherence are essential components of CR. In this context, study findings that ICT-based interventions are useful in improving medication adherence for patients with CVD, as with other chronic diseases are mounting. A recent meta-analysis of RCTs used mHealth devices for CVD patients had medication adherence as its primary outcome. According to this meta-analysis of 10 clinical trials (1-18 months of follow-up), using text messages, Bluetooth-electronic pillboxes, online messaging platforms, and conversational voice calls all enhanced drug adherence, though the degrees of improvement varied [130]. This meta-analysis was also limited because it analyzed mostly small-scale studies with short-term followups and self-reported results, but the results nevertheless suggest that mHealth tools may be useful for improving drug adherence among CVD patients (LOE of 1-).

\section{RECOMMENDATION}

- ICT-based modalities should be considered to maintain the effects of education (SOR of conditional / LOE of 1-)

\section{DISCUSSION AND CONCLUSION}

According to a 2016 Statistics Korea report [3], CVD is the second leading cause of death in Korea, and most of these events involve ACS $[1,2]$. This high mortality rate encompasses not only deaths at the time of onset, but also deaths from recurrence and complications over a short or long-term following initial onset, but unfortunately, relevant statistics are lacking in Korea. ACS clinically manifests as an acute condition, but pathologi- cally, it is actually an onset of chronic atherosclerotic vascular disease that has been slowly progressing over a long period. Therefore, even following appropriate treatment in the acute phase, patients are left with a chronic atherosclerotic vascular condition, so lifelong treatment and management is essential after discharge [4]. Many patients, moreover, have difficulty managing risk factors on their own, and continuing with appropriate levels of physical activity after discharge due to tachycardia, angina, arrhythmia, orthostatic hypotension, and reduced fitness. In particular, older patients and those with complications are faced with markedly reduced exercise capacity, which, if it persists, will allow the CAD to progress, and lead to adverse outcomes in the long-term -5 years and 10 years after onset (e.g., quality of life, recurrence, readmission, death).

A widely used treatment method worldwide, the effects, safety, and recommendations associated with CR are well established. Several countries have developed their own CPGs in consideration of their circumstances [4-9], but CR is still largely an unknown in Korea, due to a lack of awareness among patients and medical professionals, as well as a lack of relevant experts and facilities. On a positive note, in 2008, CR programs were implemented at 11 university hospitals as part of the government-led Regional Cardio-Cerebrovascular Center Project; three additional medical facilities will be added in 2019, after which 14 CR hubs will be fully operational. In addition, with the growing acceptance of CR nationwide and the introduction of CR insurance benefits, 40 medical institutions nationwide have established CR programs, and a growing number of medical institutions are preparing to offer CR. Large gaps remain among Korean medical facilities administrating CR, however, due to the lack of a standardized concept, purpose, and application. Furthermore, CPGs that document standards, principles, and methods are needed to direct medical facilities that aim to establish new CR programs, even as demands for CPGs are growing in the field.

The purpose of the CPGs proposed in this paper-the first in Korea-is to present published evidence of the need for and rationale of $\mathrm{CR}$, and the strength of recommendations that medical professionals treating CVD could make with regard to CR to their patients, with strong confidence and a sense of duty. We hope that this goal has been achieved, and that the following CPGs will 
be used to promote CR in Korea, ultimately enriching people's health and enhancing medical-economic efficiencies by markedly improving the short- and long-term outcomes of ACS (e.g., QOL, recurrence, readmission rates, reoperations, and mortality).

\section{Recommendations for Korea's CPGs}

1. Introduction of Cardiac Rehabilitation

- CR programs must be included in the treatment of ACS (SOR of strong / LOE of 1++)

- It is more appropriate to provide recommendations for the timing of exercise training than the timing of CR

- CR exercise should be initiated as early as possible following acute phase treatment (SOR of strong / LOE of 1-)

- CR programs should be comprehensive and multidisciplinary (SOR of GPP / LOE of 1-)

- Though CR programs are reported to lower health management costs in patients with CVD, high-quality Korean studies are needed to substantiate this, considering the differences of insurance systems and healthcare environments across countries (SOR of GPP / LOE of 1-)

2. Assessments of Cardiac Rehabilitation

- Individualized CR plans should be tailored to individual needs and be developed through individual assessments of patients referred to CR (SOR of strong / LOE of 1+)

- Patients referred to CR should be assessed for psychological problems, such as anxiety, depression, and stress, and should be provided with psychological interventions if abnormal findings are observed (SOR of strong / LOE of 1++)

- Cardiopulmonary exercise testing should be performed to assess patients' cardiopulmonary exercise functions, prescribe exercise, and predict outcomes (SOR of strong / LOE of 2++)

-A submaximal exercise test, such as a 6-minute walk test, should be considered when symptom-limited exercise testing is difficult (SOR of conditional / LOE of $2+$ )

- Interventions to increase self-efficacy and awareness of the need for CR are needed to increase CR participation (SOR of strong / LOE of 1++)

- Systematized automatic referrals and liaison systems should be considered to increase CR referral rates. (SOR of GPP / LOE of 2-)

- Psychological education strategies (including goal setting and self-monitoring) should be used to increase CR maintenance rates (SOR of strong / LOE of $1++)$

3. Exercise Therapy for Cardiac Rehabilitation

- CR should be initiated early after surgery, as an inpatient program for patients who undergo CABG (SOR of strong / LOE of $1++$ )

- CR exercise programs should include aerobic exercise (SOR of strong / LOE of 1++)

- High-intensity interval training may obtain better results than aerobic exercise (SOR of conditional / LOE of 1+)

- CR programs should include resistance (strengthening) exercises (SOR of conditional / LOE of 1++)

- Risk assessment and appropriate monitoring based on the risk assessment results should be performed to ensure patient safety during CR exercise programs (SOR of strong / LOE of 2++)

- Home-based CR programs may replace hospitalbased CR programs for low-risk patients (SOR of conditional / LOE of 1++)

- CR programs should also be administered to patients aged 65 years and older (SOR of strong / LOE of $1++$ )

4. Education for Secondary Prevention

- CR programs should include patient education (SOR of strong / LOE of 1++)

- The recommendations in SIGN 2017 should be largely accepted regarding educational contents (SOR of GPP / LOE of 2+) (Table 7)

- Smoking cessation interventions should be provided for patients who smoke, and continuous interventions of more than 4 weeks should be considered (SOR of strong / LOE of 1++)

- Diet programs should be designed with reference to the following guidelines (SOR of GPP / LOE of 2+) (Table 8)

- Food supplements are not recommended for secondary prevention of CAD (SOR of conditional)

- ICT-based modalities should be considered to maintain the effects of education (SOR of conditional / LOE of 1-)

Despite their clinical importance and benefits, it is 
practically difficult to design high-quality RCTs for CR, which requires a long-term behavioral correction. These CPGs were developed with reference to foreign CPGs in consideration of the latest medical technologies and circumstances in Korea, and will suffice to present standards for CR programs in Korea for the next 3-4 years. Furthermore, they will contribute to improving people's health, as well as advancing national health policies, by optimizing fitness, improving the QOL, and bettering outcomes after the discharge of patients with ACS. In the future, CR experts in relevant academic associations should continue to develop CPGs for CR in relation to heart failure, heart valve disease, congenital heart disease, arrhythmic disease, and other heart and peripheral vascular deformities.

In conclusion, these Korean CPGs for CR present levels of evidence and assess the relative strengths of the various recommendations made for the fundamental components of $\mathrm{CR}$, which should no longer be treated as alternative medicine with insufficient efficacy and evidence. We expect that CR will become an essential prescription whose benefits far outweigh its possibility for inflicting harm, and must be recommended and encouraged by physicians for most patients. Promoting CR is difficult when it is solely based on the efforts of medical institutions. Government-led strategies and support are crucial to ensuring that patients who are treated in the acute phase maintain healthy lifestyles, engage in appropriate physical activities, and adequately care for themselves. This is the fundamental purpose and direction of Korea's Act on the Prevention and Management of Cardio-Cerebrovascular Diseases.

\section{CONFLICT OF INTEREST}

No potential conflict of interest relevant to this article was reported.

\section{ACKNOWLEDGMENTS}

This study was funded by the Ministry of Health and Welfare (MOHW) as part of the Health and Medical Technology Research and Development Project of the Korea Health Industry Development Institute (KHIDI) (Project No. HC17C0063; Study period. November 1, 2018 to October 31,2018$)$. There has not been any influence from the KHIDI and National Evidence-Based Healthcare Collaborating Agency throughout the entire process of the CPGs development, and no funding has been received from other academic societies, organizations, and interest groups. The authors express their gratitude to Kyung Pyo Hong, Samsung Medical Center, Yun-Hee Kim, Department of Physical and Rehabilitation Medicine, Sungkyunkwan University School of Medicine, Sung-Bo Sim, Department of Thoracic and Cardiovascular Surgery, The Catholic University of Korea College of Medicine, WonHah Park, Department of Sports Medicine Center, Samsung Medical Center, Sungkyunkwan University School of Medicine, Kun-Sei Lee, Department of Preventive Medicine, School of Medicine, Konkuk University, Soo Young Kim, Department of Family Medicine, Kangdong Sacred Heart Hospital, Hallym University College of Medicine, Woong Ju, School of Medicine, Ewha Womans University, Sae Young Jae, Department of Sport Science, Graduate School of Urban Public Health, University of Seoul, Mi Ja Jang, Samsung Medical Center, Hye Jeong Kim, Inje University Sanggye Paik Hospital, Jung-Ho Youn, Mediplex Sejong Hospital, Song Mi Lee, Severance Hospital, Seungmin Lee, Department of Food and Nutrition, Sungshin Women's University, Jeong Ju Lee, Kyunghee University Hospital at Gangdong for their effort in expert's advices.

\section{REFERENCES}

1. Shin HY, Lee JY, Song J, Lee S, Lee J, Lim B, et al. Cause-of-death statistics in the Republic of Korea, 2014. J Korean Med Assoc 2016;59:221-32.

2. Lee SW, Kim HC, Lee HS, Suh I. Thirty-year trends in mortality from cardiovascular diseases in Korea. Korean Circ J 2015;45:202-9.

3. Korean Statistical Information Service. Cause of death statistics. Daejeon: Statistics Korea; 2016.

4. Canadian Association of Cardiac Rehabilitation. Canadian guidelines for cardiac rehabilitation and cardiovascular disease prevention: translating knowledge into action. Winnipeg: Canadian Association of Cardiac Rehabilitation; 2009.

5. Scottish Intercollegiate Guidelines Network. Cardiac rehabilitation: a national clinical guideline. Edinburgh: Scottish Intercollegiate Guidelines Network; 2017. 
6. National Institute for Health and Care Excellence. Myocardial infarction: cardiac rehabilitation and prevention of further cardiovascular disease (CG172). London: National Institute for Health and Care Excellence; 2013.

7. Smith SC Jr, Benjamin EJ, Bonow RO, Braun LT, Creager MA, Franklin BA, et al. AHA/ACCF Secondary Prevention and Risk Reduction Therapy for Patients with Coronary and other Atherosclerotic Vascular Disease: 2011 update. A guideline from the American Heart Association and American College of Cardiology Foundation. Circulation 2011;124:2458-73.

8. JCS Joint Working Group. Guidelines for rehabilitation in patients with cardiovascular disease (JCS 2012). Circ J 2014;78:2022-93.

9. Piepoli MF, Hoes AW, Agewall S, Albus C, Brotons C, Catapano AL, et al. 2016 European Guidelines on cardiovascular disease prevention in clinical practice: the Sixth Joint Task Force of the European Society of Cardiology and Other Societies on Cardiovascular Disease Prevention in Clinical Practice (constituted by representatives of 10 societies and by invited experts)Developed with the special contribution of the European Association for Cardiovascular Prevention \& Rehabilitation (EACPR). Eur Heart J 2016;37:2315-81.

10. Steering Committee for Clinical Practice Guideline. Korean Appraisal of Guidelines for Research \& Evaluation II. Seoul: Ministry of Health \& Welfare, Korean Academy of Medical Science; 2010.

11. Cardiac Rehabilitation Guideline Panel. Cardiac rehabilitation as secondary prevention. Am Fam Physician 1995;52:2257-64.

12. Balady GJ, Ades PA, Bittner VA, Franklin BA, Gordon NF, Thomas RJ, et al. Referral, enrollment, and delivery of cardiac rehabilitation/secondary prevention programs at clinical centers and beyond: a presidential advisory from the American Heart Association. Circulation 2011;124:2951-60.

13. Perk J, De Backer G, Gohlke H, Graham I, Reiner Z, Verschuren M, et al. European Guidelines on cardiovascular disease prevention in clinical practice (version 2012). The Fifth Joint Task Force of the European Society of Cardiology and Other Societies on Cardiovascular Disease Prevention in Clinical Practice (constituted by representatives of nine societies and by invited experts). Eur Heart J 2012;33:1635701.

14. Anderson L, Oldridge N, Thompson DR, Zwisler AD, Rees K, Martin N, et al. Exercise-based cardiac rehabilitation for coronary heart disease: Cochrane systematic review and meta-analysis. J Am Coll Cardiol 2016;67:1-12.

15. Sumner J, Harrison A, Doherty P. The effectiveness of modern cardiac rehabilitation: a systematic review of recent observational studies in non-attenders versus attenders. PLoS One 2017;12:e0177658.

16. Goodwin L, Ostuzzi G, Khan N, Hotopf MH, MossMorris R. Can we identify the active ingredients of behaviour change interventions for coronary heart disease patients? A systematic review and metaanalysis. PLoS One 2016;11:e153271.

17. Kim C, Kim DY, Moon CJ. Prognostic influences of cardiac rehabilitation in Korean acute myocardial infarction patients. Ann Rehabil Med 2011;35:37580.

18. Anderson L, Taylor RS. Cardiac rehabilitation for people with heart disease: an overview of Cochrane systematic reviews. Cochrane Database Syst Rev 2014;(12):CD011273.

19. Shepherd CW, While AE. Cardiac rehabilitation and quality of life: a systematic review. Int J Nurs Stud 2012;49:755-71.

20. Abell B, Glasziou P, Hoffmann T. The contribution of individual exercise training components to clinical outcomes in randomised controlled trials of cardiac rehabilitation: a systematic review and meta-regression. Sports Med Open 2017;3:19.

21. van Halewijn G, Deckers J, Tay HY, van Domburg R, Kotseva K, Wood D. Lessons from contemporary trials of cardiovascular prevention and rehabilitation: a systematic review and meta-analysis. Int J Cardiol 2017;232:294-303.

22. Chan E, Giallauria F, Vigorito C, Smart NA. Exercise training in heart failure patients with preserved ejection fraction: a systematic review and meta-analysis. Monaldi Arch Chest Dis 2016;86:759.

23. Pandey A, Parashar A, Kumbhani D, Agarwal S, Garg J, Kitzman D, et al. Exercise training in patients with heart failure and preserved ejection fraction: metaanalysis of randomized control trials. Circ Heart Fail 2015;8:33-40. 
24. Haykowsky M, Scott J, Esch B, Schopflocher D, Myers J, Paterson I, et al. A meta-analysis of the effects of exercise training on left ventricular remodeling following myocardial infarction: start early and go longer for greatest exercise benefits on remodeling. Trials 2011;12:92.

25. Zhang YM, Lu Y, Tang Y, Yang D, Wu HF, Bian ZP, et al. The effects of different initiation time of exercise training on left ventricular remodeling and cardiopulmonary rehabilitation in patients with left ventricular dysfunction after myocardial infarction. Disabil Rehabil 2016;38:268-76.

26. Oldridge N. Exercise-based cardiac rehabilitation in patients with coronary heart disease: meta-analysis outcomes revisited. Future Cardiol 2012;8:729-51.

27. Kim C, Bang HJ, Kim JH, Sohn MK, Yang CY, Lee SG, et al. Recommendations for establishing cardiac rehabilitation programs: facility, equipment and staff. The Korean Society of Cardiac Rehabilitation (KSCR) position statement. J Korean Acad Rehabil Med 2010; 34:491-7.

28. Jones J, Buckley JP, Furze G, Doherty P, Speck L, Connolly S, et al. The BACPR standards and core components for cardiovascular prevention and rehabilitation 2012. London: British Association for Cardiovascular Prevention and Rehabilitation; 2012.

29. Clark AM, Catto S, Bowman G, Macintyre PD. Design matters in secondary prevention: individualization and supervised exercise improves the effectiveness of cardiac rehabilitation. Eur J Cardiovasc Prev Rehabil 2011;18:761-9.

30. Coulter A, Entwistle VA, Eccles A, Ryan S, Shepperd S, Perera R. Personalised care planning for adults with chronic or long-term health conditions. Cochrane Database Syst Rev 2015;(3):CD010523.

31. Hill K, Walwyn R, Camidge D, Murray J, Meads D, Reynolds G, et al. A randomized feasibility trial of a new lifestyle referral assessment versus usual assessment in an acute cardiology setting. J Cardiovasc Nurs 2016;31:507-516.

32. Weibel L, Massarotto P, Hediger H, Mahrer-Imhof R. Early education and counselling of patients with acute coronary syndrome. A pilot study for a randomized controlled trial. Eur J Cardiovasc Nurs 2016; 15:213-22.

33. Fors A, Swedberg K, Ulin K, Wolf A, Ekman I. Effects of person-centred care after an event of acute coronary syndrome: two-year follow-up of a randomized controlled trial. Int J Cardiol 2017;249:42-7.

34. Lesperance F, Frasure-Smith N. Depression in patients with cardiac disease: a practical review. J Psychosom Res 2000;48:379-91.

35. Carney RM, Freedland KE. Depression, mortality, and medical morbidity in patients with coronary heart disease. Biol Psychiatry 2003;54:241-7.

36. Frasure-Smith N, Lesperance F. Recent evidence linking coronary heart disease and depression. Can J Psychiatry 2006;51:730-7.

37. Gehi A, Haas D, Pipkin S, Whooley MA. Depression and medication adherence in outpatients with coronary heart disease: findings from the Heart and Soul Study. Arch Intern Med 2005;165:2508-13.

38. Ades PA, Waldmann ML, McCann WJ, Weaver SO. Predictors of cardiac rehabilitation participation in older coronary patients. Arch Intern Med 1992;152: 1033-5.

39. Glazer KM, Emery CF, Frid DJ, Banyasz RE. Psychological predictors of adherence and outcomes among patients in cardiac rehabilitation. J Cardiopulm Rehabil 2002;22:40-6.

40. Frasure-Smith N, Lesperance F, Gravel G, Masson A, Juneau M, Talajic M, et al. Depression and healthcare costs during the first year following myocardial infarction. J Psychosom Res 2000;48:471-8.

41. Shibeshi WA, Young-Xu Y, Blatt CM. Anxiety worsens prognosis in patients with coronary artery disease. J Am Coll Cardiol 2007;49:2021-7.

42. Murphy BM, Higgins RO, Jackson AC. Anxiety, depression, and psychological adjustment after an acute cardiac event. In: Alvarenga ME, Byrne DG, editors. Handbook of psychocardiology. Singapore: Springer; 2015. p. 1-21.

43. Richards SH, Anderson L, Jenkinson CE, Whalley B, Rees K, Davies P, et al. Psychological interventions for coronary heart disease. Cochrane Database Syst Rev 2017;4:CD002902.

44. Klainin-Yobas P, Ng SH, Stephen PDM, Lau Y. Efficacy of psychosocial interventions on psychological outcomes among people with cardiovascular diseases: a systematic review and meta-analysis. Patient Educ Couns 2016;99:512-21.

45. von Kanel R, Barth J, Princip M, Meister-Langraf RE, 
Schmid JP, Znoj H, et al. Early psychological counseling for the prevention of posttraumatic stress induced by acute coronary syndrome: the MI-SPRINT randomized controlled trial. Psychother Psychosom 2018;87:75-84.

46. American College of Sports Medicine. ACSM's guidelines for exercise testing and prescription. 9th ed. Philadelphia, PA: Lippincott Williams \& Wilkins; 2013.

47. American Association of Cardiovascular \& Pulmonary Rehabilitation. Guidelines for Cardiac Rehabilitation and Secondary Prevention Programs. Champaign, IL: Human Kinetics; 2013.

48. Balady GJ, Leitschuh ML, Jacobs AK, Merrell D, Weiner DA, Ryan TJ. Safety and clinical use of exercise testing one to three days after percutaneous transluminal coronary angioplasty. Am J Cardiol 1992;69:1259-64.

49. Horgan J, Bethell H, Carson P, Davidson C, Julian D, Mayou RA, et al. Working party report on cardiac rehabilitation. Br Heart J 1992;67:412-8.

50. Goble AJ, Worcester MC. Best practice guidelines for cardiac rehabilitation and secondary prevention. Melbourne, Australia: Department of Human Services; 1999.

51. Wright DJ, Khan KM, Gossage EM, Saltissi S. Assessment of a low-intensity cardiac rehabilitation programme using the six-minute walk test. Clin Rehabil 2001;15:119-24.

52. Tallaj JA, Sanderson B, Breland J, Adams C, Schumann C, Bittner V. Assessment of functional outcomes using the 6-minute walk test in cardiac rehabilitation: comparison of patients with and without left ventricular dysfunction. J Cardiopulm Rehabil 2001;21:221-4.

53. Verrill DE, Barton C, Beasley W, Lippard M, King CN. Six-minute walk performance and quality of life comparisons in North Carolina cardiac rehabilitation programs. Heart Lung 2003;32:41-51.

54. Bellet RN, Adams L, Morris NR. The 6-minute walk test in outpatient cardiac rehabilitation: validity, reliability and responsiveness. A systematic review. Physiotherapy 2012;98:277-86.

55. Nogueira PA, Leal AC, Pulz C, Nogueira ID, Filho JA. Clinical reliability of the 6 minute corridor walk test performed within a week of a myocardial infarction.
Int Heart J 2006;47:533-40.

56. Hanson LC, McBurney H, Taylor NF. The retest reliability of the six-minute walk test in patients referred to a cardiac rehabilitation programme. Physiother Res Int 2012;17:55-61.

57. Harris KM, Anderson DR, Landers JD, Emery CF. Utility of walk tests in evaluating functional status among participants in an outpatient cardiac rehabilitation program. J Cardiopulm Rehabil Prev 2017;37: 329-33.

58. Goto Y. Current state of cardiac rehabilitation in Japan. Prog Cardiovasc Dis 2014;56:557-62.

59. Im HW, Baek S, Jee S, Ahn JM, Park MW, Kim WS. Barriers to outpatient hospital-based cardiac rehabilitation in Korean patients with acute coronary syndrome. Ann Rehabil Med 2018;42:154-65.

60. Ades PA, Keteyian SJ, Wright JS, Hamm LF, Lui K, Newlin K, et al. Increasing cardiac rehabilitation participation from $20 \%$ to $70 \%$ : a road map from the Million Hearts Cardiac Rehabilitation Collaborative. Mayo Clin Proc 2017;92:234-42.

61. Karmali KN, Davies P, Taylor F, Beswick A, Martin N, Ebrahim S. Promoting patient uptake and adherence in cardiac rehabilitation. Cochrane Database Syst Rev 2014;(6):CD007131.

62. Cossette S, Frasure-Smith N, Dupuis J, Juneau M, Guertin MC. Randomized controlled trial of tailored nursing interventions to improve cardiacrehabilitation enrollment. Nurs Res 2012;61:111-20.

63. Hillebrand T, Frodermann H, Lehr D, Wirth A. Increased participation in coronary groups by means of an outpatient care program. Herz Kreislauf 1995; 27:346-9.

64. Jolly K, Bradley F, Sharp S, Smith H, Thompson S, Kinmonth AL, et al. Randomised controlled trial of follow up care in general practice of patients with myocardial infarction and angina: final results of the Southampton heart integrated care project (SHIP). The SHIP Collaborative Group. BMJ 1999;318:70611.

65. Price JA. A pilot trial of a coaching intervention designed to increase women's attendance at cardiac rehabilitation intake [dissertation]. Toronto, Canada: University of Toronto; 2012.

66. Pack QR, Mansour M, Barboza JS, Hibner BA, Mahan MG, Ehrman JK, et al. An early appointment 
to outpatient cardiac rehabilitation at hospital discharge improves attendance at orientation: a randomized, single-blind, controlled trial. Circulation 2013;127:349-55.

67. Wyer S, Earll L, Joseph S, Harrison J, Giles M, Johnston $\mathrm{M}$. Increasing attendance at a cardiac rehabilitation programme: an intervention study using the Theory of Planned Behaviour. Coron Health Care 2001;5:154-9.

68. Arrigo I, Brunner-LaRocca H, Lefkovits M, Pfisterer M, Hoffmann A. Comparative outcome one year after formal cardiac rehabilitation: the effects of a randomized intervention to improve exercise adherence. Eur J Cardiovasc Prev Rehabil 2008;15:306-11.

69. Duncan KA, Pozehl B. Staying on course: the effects of an adherence facilitation intervention on home exercise participation. Prog Cardiovasc Nurs 2002;17: $59-65,71$.

70. Sniehotta FF, Scholz U, Schwarzer R. Action plans and coping plans for physical exercise: a longitudinal intervention study in cardiac rehabilitation. Br J Health Psychol 2006;11(Pt 1):23-37.

71. Reid RD, Morrin LI, Higginson LA, Wielgosz A, Blanchard C, Beaton LJ, et al. Motivational counselling for physical activity in patients with coronary artery disease not participating in cardiac rehabilitation. Eur J Prev Cardiol 2012;19:161-6.

72. Grace SL, Midence L, Oh P, Brister S, Chessex C, Stewart DE, et al. Cardiac rehabilitation program adherence and functional capacity among women: a randomized controlled trial. Mayo Clin Proc 2016;91: 140-8.

73. Lynggaard V, Nielsen CV, Zwisler AD, Taylor RS, May $O$. The patient education: learning and coping strategies. Improves adherence in cardiac rehabilitation (LC-REHAB): a randomised controlled trial. Int J Cardiol 2017;236:65-70.

74. Grace SL, Chessex C, Arthur H, Chan S, Cyr C, Dafoe $\mathrm{W}$, et al. Systematizing inpatient referral to cardiac rehabilitation 2010: Canadian Association of Cardiac Rehabilitation and Canadian Cardiovascular Society joint position paper endorsed by the Cardiac Care Network of Ontario. Can J Cardiol 2011;27:192-9.

75. Taylor RS, Brown A, Ebrahim S, Jolliffe J, Noorani H, Rees K, et al. Exercise-based rehabilitation for patients with coronary heart disease: systematic review and meta-analysis of randomized controlled trials. Am J Med 2004;116:682-92.

76. van den Berg-Emons RJ, Bussmann JB, Balk AH, Stam HJ. Factors associated with the level of movement-related everyday activity and quality of life in people with chronic heart failure. Phys Ther 2005;85: 1340-8.

77. ter Hoeve N, Huisstede BM, Stam HJ, van Domburg RT, Sunamura M, van den Berg-Emons RJ. Does cardiac rehabilitation after an acute cardiac syndrome lead to changes in physical activity habits? Systematic review. Phys Ther 2015;95:167-79.

78. Cha S, Park JJ, Kim S, Ahn HY, Han K, Lee Y, et al. Need for systematic efforts to modify health-related behaviors after acute myocardial infarction in Korea. Circ J 2018;82:2523-9.

79. Aldcroft SA, Taylor NF, Blackstock FC, O'Halloran PD. Psychoeducational rehabilitation for health behavior change in coronary artery disease: a systematic review of controlled trials. J Cardiopulm Rehabil Prev 2011;31:273-81.

80. Tingstrom PR, Kamwendo K, Bergdahl B. Effects of a problem-based learning rehabilitation programme on quality of life in patients with coronary artery disease. Eur J Cardiovasc Nurs 2005;4:324-30.

81. Carlson JJ, Johnson JA, Franklin BA, VanderLaan RL. Program participation, exercise adherence, cardiovascular outcomes, and program cost of traditional versus modified cardiac rehabilitation. Am J Cardiol 2000;86:17-23.

82. Izawa KP, Watanabe S, Omiya K, Hirano Y, Oka K, Osada N, et al. Effect of the self-monitoring approach on exercise maintenance during cardiac rehabilitation: a randomized, controlled trial. Am J Phys Med Rehabil 2005;84:313-21.

83. Fournier M, Radel R, Bailly L, Pradier C, Fabre R, Fuch A, et al. "As du Coeur" study: a randomized controlled trial on physical activity maintenance in cardiovascular patients. BMC Cardiovasc Disord 2018;18:77.

84. Ter Hoeve N, Sunamura M, Stam HJ, Boersma E, Geleijnse ML, van Domburg RT, et al. Effects of two behavioral cardiac rehabilitation interventions on physical activity: a randomized controlled trial. Int J Cardiol 2018;255:221-8.

85. Kulik A, Ruel M, Jneid H, Ferguson TB, Hiratzka LF, Ikonomidis JS, et al. Secondary prevention after cor- 
onary artery bypass graft surgery: a scientific statement from the American Heart Association. Circulation 2015;131:927-64.

86. Hansen D, Dendale P, Leenders M, Berger J, Raskin A, Vaes J, et al. Reduction of cardiovascular event rate: different effects of cardiac rehabilitation in CABG and PCI patients. Acta Cardiol 2009;64:639-44.

87. Hedback B, Perk J, Hornblad M, Ohlsson U. Cardiac rehabilitation after coronary artery bypass surgery: 10 -year results on mortality, morbidity and readmissions to hospital. J Cardiovasc Risk 2001;8:153-8.

88. Aldahash R, Al Dera HS. Physical therapy program improves the physiological impact towards better quality of life and low cardiac risk factors in patients following coronary artery bypass grafting: systematic review. Acta Medica Int 2016;3:185-95.

89. Kim SY, Oh JK, Youn JH, Kim YJ. The effect of cardiac rehabilitation I phase program on physical capacity after coronary artery bypass graft surgery. Korean J Sports Med 2012;30:85-91.

90. Hamm LF, Sanderson BK, Ades PA, Berra K, Kaminsky LA, Roitman JL, et al. Core competencies for cardiac rehabilitation/secondary prevention professionals: 2010 update. Position statement of the American Association of Cardiovascular and Pulmonary Rehabilitation. J Cardiopulm Rehabil Prev 2011; 31:2-10.

91. Anderson L, Thompson DR, Oldridge N, Zwisler $\mathrm{AD}$, Rees $\mathrm{K}$, Martin N, et al. Exercise-based cardiac rehabilitation for coronary heart disease. Cochrane Database Syst Rev 2016;(1):CD001800.

92. Brown A, Taylor R, Noorani H, Stone J, Skidmore B. Exercise-based cardiac rehabilitation programs for coronary artery disease: a systematic clinical and economic review. Ottawa, Canada: Canadian Coordinating Office for Health Technology Assessment; 2003.

93. Chen YC, Tsai JC, Liou YM, Chan P. Effectiveness of endurance exercise training in patients with coronary artery disease: a meta-analysis of randomised controlled trials. Eur J Cardiovasc Nurs 2017;16:397408.

94. Liou K, Ho S, Fildes J, Ooi SY. High intensity interval versus moderate intensity continuous training in patients with coronary artery disease: a meta-analysis of physiological and clinical parameters. Heart Lung
Circ 2016;25:166-74.

95. Choi HY, Han HJ, Choi JW, Jung HY, Joa KL. Superior effects of high-intensity interval training compared to conventional therapy on cardiovascular and psychological aspects in myocardial infarction. Ann Rehabil Med 2018;42:145-53.

96. Kim C, Choi HE, Lim MH. Effect of high interval training in acute myocardial infarction patients with drug-eluting stent. Am J Phys Med Rehabil 2015; 94(10 Suppl 1):879-86.

97. Williams MA, Haskell WL, Ades PA, Amsterdam EA, Bittner V, Franklin BA, et al. Resistance exercise in individuals with and without cardiovascular disease: 2007 update: a scientific statement from the American Heart Association Council on Clinical Cardiology and Council on Nutrition, Physical Activity, and Metabolism. Circulation 2007;116:572-84.

98. Hollings M, Mavros Y, Freeston J, Fiatarone Singh $M$. The effect of progressive resistance training on aerobic fitness and strength in adults with coronary heart disease: a systematic review and meta-analysis of randomized controlled trials. Eur J Prev Cardiol 2017;24:1242-59.

99. Karagiannis C, Savva C, Mamais I, Efstathiou M, Monticone M, Xanthos T. Eccentric exercise in ischemic cardiac patients and functional capacity: a systematic review and meta-analysis of randomized controlled trials. Ann Phys Rehabil Med 2017;60:5864.

100. Xanthos PD, Gordon BA, Kingsley MI. Implementing resistance training in the rehabilitation of coronary heart disease: a systematic review and meta-analysis. Int J Cardiol 2017;230:493-508.

101. Kavanagh T, Mertens DJ, Hamm LF, Beyene J, Kennedy J, Corey P, et al. Prediction of long-term prognosis in 12169 men referred for cardiac rehabilitation. Circulation 2002;106:666-71.

102. Kavanagh T, Mertens DJ, Hamm LF, Beyene J, Kennedy J, Corey P, et al. Peak oxygen intake and cardiac mortality in women referred for cardiac rehabilitation. J Am Coll Cardiol 2003;42:2139-43.

103. Mark DB, Hlatky MA, Harrell FE Jr, Lee KL, Califf RM, Pryor DB. Exercise treadmill score for predicting prognosis in coronary artery disease. Ann Intern Med 1987;106:793-800.

104. Mark DB, Shaw L, Harrell FE Jr, Hlatky MA, Lee KL, 
Bengtson JR, et al. Prognostic value of a treadmill exercise score in outpatients with suspected coronary artery disease. N Engl J Med 1991;325:849-53.

105. Vanhees L, Fagard R, Thijs L, Staessen J, Amery A. Prognostic significance of peak exercise capacity in patients with coronary artery disease. J Am Coll Cardiol 1994;23:358-63.

106. Kokkinos P, Myers J, Kokkinos JP, Pittaras A, Narayan $\mathrm{P}$, Manolis A, et al. Exercise capacity and mortality in black and white men. Circulation 2008;117:614-22.

107. Fletcher GF, Balady GJ, Amsterdam EA, Chaitman B, Eckel R, Fleg J, et al. Exercise standards for testing and training: a statement for healthcare professionals from the American Heart Association. Circulation 2001;104:1694-740.

108. Kim C, Moon CJ, Lim MH. Safety of monitoring exercise for early hospital-based cardiac rehabilitation. Ann Rehabil Med 2012;36:262-7.

109. Claes J, Buys R, Budts W, Smart N, Cornelissen VA. Longer-term effects of home-based exercise interventions on exercise capacity and physical activity in coronary artery disease patients: a systematic review and meta-analysis. Eur J Prev Cardiol 2017;24:24456.

110. Anderson L, Sharp GA, Norton RJ, Dalal H, Dean SG, Jolly K, et al. Home-based versus centre-based cardiac rehabilitation. Cochrane Database Syst Rev 2017;6:CD007130.

111. Huang K, Liu W, He D, Huang B, Xiao D, Peng Y, et al. Telehealth interventions versus center-based cardiac rehabilitation of coronary artery disease: a systematic review and meta-analysis. Eur J Prev Cardiol 2015;22:959-71.

112. McClure T, Haykowsky MJ, Schopflocher D, Hsu ZY, Clark AM. Home-based secondary prevention programs for patients with coronary artery disease: a meta-analysis of effects on anxiety. J Cardiopulm Rehabil Prev 2013;33:59-67.

113. Heron N, Kee F, Donnelly M, Cardwell C, Tully MA, Cupples ME. Behaviour change techniques in homebased cardiac rehabilitation: a systematic review. $\mathrm{Br}$ J Gen Pract 2016;66:e747-57.

114. Lee YH, Hur SH, Sohn J, Lee HM, Park NH, Cho YK, et al. Impact of home-based exercise training with wireless monitoring on patients with acute coronary syndrome undergoing percutaneous coronary inter- vention. J Korean Med Sci 2013;28:564-8.

115. Chung H, Ko H, Thap T, Jeong C, Noh SE, Yoon KH, et al. Smartphone-based cardiac rehabilitation program: feasibility study. PLoS One 2016;11:e0161268.

116. Lee H, Chung H, Ko H, Jeong C, Noh SE, Kim C, et al. Dedicated cardiac rehabilitation wearable sensor and its clinical potential. PLoS One 2017;12:e0187108.

117. Rich MW, Bosner MS, Chung MK, Shen J, McKenzie JP. Is age an independent predictor of early and late mortality in patients with acute myocardial infarction? Am J Med 1992;92:7-13.

118. Ades PA, Savage PD, Tischler MD, Poehlman ET, Dee J, Niggel J. Determinants of disability in older coronary patients. Am Heart J 2002;143:151-6.

119. Pasquali SK, Alexander KP, Peterson ED. Cardiac rehabilitation in the elderly. Am Heart J 2001;142:74855.

120. Audelin MC, Savage PD, Ades PA. Exercise-based cardiac rehabilitation for very old patients $(>$ or $=75$ years): focus on physical function. J Cardiopulm Rehabil Prev 2008;28:163-73.

121. Yamamoto S, Hotta K, Ota E, Mori R, Matsunaga A. Effects of resistance training on muscle strength, exercise capacity, and mobility in middle-aged and elderly patients with coronary artery disease: a metaanalysis. J Cardiol 2016;68:125-34.

122. Kim JH. Effects of cardiac rehabilitation in elderly patients after myocardial infarction. J Korea AcadInd Coop Soc 2016;17:464-71.

123. British Association for Cardiovascular Prevention and Rehabilitation. The BACPR standards and core components for cardiovascular disease prevention and rehabilitation 2017. London: British Cardiovascular Society; 2017.

124. Ferrier S, Blanchard CM, Vallis M, Giacomantonio N. Behavioural interventions to increase the physical activity of cardiac patients: a review. Eur J Cardiovasc Prev Rehabil 2011;18:15-32.

125. Tierney S, Mamas M, Skelton D, Woods S, Rutter MK, Gibson M, et al. What can we learn from patients with heart failure about exercise adherence? A systematic review of qualitative papers. Health Psychol 2011;30:401-10.

126. Anderson L, Brown JP, Clark AM, Dalal H, Rossau HK, Bridges C, et al. Patient education in the management of coronary heart disease. Cochrane Data- 
base Syst Rev 2017;6:CD008895.

127. Nieuwlaat R, Wilczynski N, Navarro T, Hobson N, Jeffery R, Keepanasseril A, et al. Interventions for enhancing medication adherence. Cochrane Database Syst Rev 2014;(11):CD000011.

128. Chase JA, Bogener JL, Ruppar TM, Conn VS. The effectiveness of medication adherence interventions among patients with coronary artery disease: a meta-analysis. J Cardiovasc Nurs 2016;31:357-66.

129. Adler AJ, Martin N, Mariani J, Tajer CD, Owolabi OO, Free $\mathrm{C}$, et al. Mobile phone text messaging to improve medication adherence in secondary prevention of cardiovascular disease. Cochrane Database Syst Rev 2017;4:CD011851.

130. Gandapur Y, Kianoush S, Kelli HM, Misra S, Urrea B, Blaha MJ, et al. The role of mHealth for improving medication adherence in patients with cardiovascular disease: a systematic review. Eur Heart J Qual Care Clin Outcomes 2016;2:237-44.

131. Gandhi S, Chen S, Hong L, Sun K, Gong E, Li C, et al. Effect of mobile health interventions on the secondary prevention of cardiovascular disease: systematic review and meta-analysis. Can J Cardiol 2017;33:21931.

132. Zullig LL, Ramos K, Bosworth HB. Improving medication adherence in coronary heart disease. Curr Cardiol Rep 2017;19:113.

133. Barth J, Jacob T, Daha I, Critchley JA. Psychosocial interventions for smoking cessation in patients with coronary heart disease. Cochrane Database Syst Rev 2015;(7):CD006886.

134. Kotb A, Hsieh S, Wells GA. The effect of telephone support interventions on coronary artery disease (CAD) patient outcomes during cardiac rehabilitation: a systematic review and meta-analysis. PLoS One 2014;9:e96581.

135. Rigotti NA, Clair C, Munafo MR, Stead LF. Interventions for smoking cessation in hospitalised patients. Cochrane Database Syst Rev 2012;(5):CD001837.

136. Rice VH, Hartmann-Boyce J, Stead LF. Nursing interventions for smoking cessation. Cochrane Database Syst Rev 2013;(8):CD001188.

137. Stead LF, Koilpillai P, Lancaster T. Additional behavioural support as an adjunct to pharmacotherapy for smoking cessation. Cochrane Database Syst Rev 2015;(10):CD009670.
138. Franck C, Filion KB, Eisenberg MJ. Smoking cessation in patients with acute coronary syndrome. Am J Cardiol 2018;121:1105-11.

139. Eckel RH, Jakicic JM, Ard JD, de Jesus JM, Houston Miller N, Hubbard VS, et al. 2013 AHA/ACC guideline on lifestyle management to reduce cardiovascular risk: a report of the American College of Cardiology/ American Heart Association Task Force on Practice Guidelines. Circulation 2014;129(25 Suppl 2):S76-99.

140. National Heart Foundation of Australia, Cardiac Society of Australia and New Zealand. Reducing risk in heart disease: an expert guide to clinical practice for secondary prevention of coronary heart disease. Melbourne, Australia: National Heart Foundation of Australia, Cardiac Society of Australia and New Zealand; 2002

141. Committee of Clinical Practice Guideline of the Korean Society of Lipid and Atherosclerosis. Korean Guidelines for the management of dyslipidemia. 4th ed. Seoul: Korean Society of Lipid and Atherosclerosis; 2018.

142. The Committee of Hypertension Clinical Guideline of Korean Academy of Medical Sciences. Evidencebased recommendations for hypertension in primary care. Seoul: Korean Academy of Medical Sciences, Korea Centers for Disease Control \& Prevention; 2018.

143. Sofi F, Abbate R, Gensini GF, Casini A. Accruing evidence on benefits of adherence to the Mediterranean diet on health: an updated systematic review and meta-analysis. Am J Clin Nutr 2010;92:1189-96.

144. Estruch R, Ros E, Salas-Salvado J, Covas MI, Corella D, Aros F, et al. Primary prevention of cardiovascular disease with a Mediterranean diet. N Engl J Med 2013;368:1279-90.

145. Estruch R, Ros E, Salas-Salvado J, Covas MI, Corella D, Aros F, et al. Primary Prevention of Cardiovascular Disease with a Mediterranean Diet Supplemented with Extra-Virgin Olive Oil or Nuts. N Engl J Med 2018;378:e34.

146. de Lorgeril M, Salen P, Martin JL, Monjaud I, Delaye J, Mamelle N. Mediterranean diet, traditional risk factors, and the rate of cardiovascular complications after myocardial infarction: final report of the Lyon Diet Heart Study. Circulation 1999;99:779-85.

147. Singh RB, Rastogi SS, Verma R, Laxmi B, Singh R, Ghosh S, et al. Randomised controlled trial of car- 
dioprotective diet in patients with recent acute myocardial infarction: results of one year follow up. BMJ 1992;304:1015-9.

148. McGowan MP, Proulx S. Nutritional supplements and serum lipids: does anything work? Curr Atheroscler Rep 2009;11:470-6.

149. Abdelhamid AS, Brown TJ, Brainard JS, Biswas P, Thorpe GC, Moore HJ, et al. Omega-3 fatty acids for the primary and secondary prevention of cardiovascular disease. Cochrane Database Syst Rev 2018; 7:CD003177.

150. Kotwal S, Jun M, Sullivan D, Perkovic V, Neal B. Omega 3 Fatty acids and cardiovascular outcomes: systematic review and meta-analysis. Circ Cardiovasc Qual Outcomes 2012;5:808-18.

151. Rizos EC, Elisaf MS. Does supplementation with omega-3 PUFAs add to the prevention of cardiovascular disease? Curr Cardiol Rep 2017;19:47.

152. Enns JE, Yeganeh A, Zarychanski R, Abou-Setta AM, Friesen C, Zahradka P, et al. The impact of omega-3 polyunsaturated fatty acid supplementation on the incidence of cardiovascular events and complications in peripheral arterial disease: a systematic review and meta-analysis. BMC Cardiovasc Disord 2014;14:70.

153. Gong J, Qin X, Yuan F, Hu M, Chen G, Fang K, et al. Efficacy and safety of sugarcane policosanol on dyslipidemia: A meta-analysis of randomized controlled trials. Mol Nutr Food Res 2018;62.

154. Marazzi G, Campolongo G, Pelliccia F, Quattrino S, Vitale C, Cacciotti L, et al. Comparison of low-dose statin versus low-dose statin + Armolipid plus in high-intensity statin-intolerant patients with a previous coronary event and percutaneous coronary intervention (ADHERENCE Trial). Am J Cardiol 2017; 120:893-7.

155. Xu K, Liu X, Li Y, Wang Y, Zang H, Guo L, et al. Safety and efficacy of policosanol in patients with high ontreatment platelet reactivity after drug-eluting stent implantation: two-year follow-up results. Cardiovasc Ther 2016;34:337-42.

156. Berthold HK, Unverdorben S, Degenhardt R, Bulitta M, Gouni-Berthold I. Effect of policosanol on lipid levels among patients with hypercholesterolemia or combined hyperlipidemia: a randomized controlled trial. JAMA 2006;295:2262-9.
157. Ye Y, Li J, Yuan Z. Effect of antioxidant vitamin supplementation on cardiovascular outcomes: a metaanalysis of randomized controlled trials. PLoS One 2013;8:e56803.

158. Lee IM, Cook NR, Gaziano JM, Gordon D, Ridker PM, Manson JE, et al. Vitamin E in the primary prevention of cardiovascular disease and cancer: the Women's Health Study: a randomized controlled trial. JAMA 2005;294:56-65.

159. Lee IM, Cook NR, Manson JE, Buring JE, Hennekens $\mathrm{CH}$. Beta-carotene supplementation and incidence of cancer and cardiovascular disease: the Women's Health Study. J Natl Cancer Inst 1999;91:2102-6.

160. Loffredo L, Perri L, Di Castelnuovo A, Iacoviello L, De Gaetano G, Violi F. Supplementation with vitamin $\mathrm{E}$ alone is associated with reduced myocardial infarction: a meta-analysis. Nutr Metab Cardiovasc Dis 2015;25:354-63.

161. Myung SK, Ju W, Cho B, Oh SW, Park SM, Koo BK, et al. Efficacy of vitamin and antioxidant supplements in prevention of cardiovascular disease: systematic review and meta-analysis of randomized controlled trials. BMJ 2013;346:f10.

162. World Health Organization. mHealth: new horizons for health through mobile technologies. Geneva, Switzerland: World Health Organization; 2011.

163. Poushter, J. Smartphone ownership and internet usage continues to climb in emerging economies. Washington, DC: Pew Research Center; 2016.

164. Statista Inc. Mobile Internet - Statistics \& Facts [Internet]. New York, NY: Statista Inc.; 2018 [cited 2019 Jun 1]. Available from: https://www.statista.com/ topics/779/mobile-internet/.

165. Statista Inc. Number of mHealth app downloads worldwide from 2013 to 2017 (in billions) [Internet]. New York, NY: Statista Inc.; c2019 [cited 2019 Jun 1]. Available from: https://www.statista.com/statistics/625034/mobile-health-app-downloads/.

166. Clark RA, Conway A, Poulsen V, Keech W, Tirimacco $\mathrm{R}$, Tideman P. Alternative models of cardiac rehabilitation: a systematic review. Eur J Prev Cardiol 2015; 22:35-74.

167. Maddison R, Pfaeffli L, Whittaker R, Stewart R, Kerr A, Jiang $\mathrm{Y}$, et al. A mobile phone intervention increases physical activity in people with cardiovascular disease: Results from the HEART randomized 
controlled trial. Eur J Prev Cardiol 2015;22:701-9.

168. Butler L, Furber S, Phongsavan P, Mark A, Bauman A. Effects of a pedometer-based intervention on physical activity levels after cardiac rehabilitation: a randomized controlled trial. J Cardiopulm Rehabil Prev 2009;29:105-14.

169. Verheijden Klompstra L, Jaarsma T, Stromberg A. Exergaming in older adults: a scoping review and implementation potential for patients with heart failure. Eur J Cardiovasc Nurs 2014;13:388-98.

170. Devi R, Powell J, Singh S. A web-based program improves physical activity outcomes in a primary care angina population: randomized controlled trial. J Med Internet Res 2014;16:e186.

171. Lear SA, Singer J, Banner-Lukaris D, Horvat D, Park JE, Bates J, et al. Randomized trial of a virtual cardiac rehabilitation program delivered at a distance via the Internet. Circ Cardiovasc Qual Outcomes 2014;7:9529.

172. Reid RD, Morrin LI, Beaton LJ, Papadakis S, Kocourek J, McDonnell L, et al. Randomized trial of an internet-based computer-tailored expert system for physical activity in patients with heart disease. Eur J Prev Cardiol 2012;19:1357-64.

173. Coorey GM, Neubeck L, Mulley J, Redfern J. Effectiveness, acceptability and usefulness of mobile applications for cardiovascular disease self-man- agement: systematic review with meta-synthesis of quantitative and qualitative data. Eur J Prev Cardiol 2018;25:505-21.

174. Ho PM, Bryson CL, Rumsfeld JS. Medication adherence: its importance in cardiovascular outcomes. Circulation 2009;119:3028-35.

175. Korean Nutrition Society. (2015) Dietary reference intakes for Koreans. Seoul: Korean Nutrition Society; 2016.

176. He FJ, MacGregor GA. Effect of modest salt reduction on blood pressure: a meta-analysis of randomized trials. Implications for public health. J Hum Hypertens 2002;16:761-70.

177. Sacks FM, Svetkey LP, Vollmer WM, Appel LJ, Bray GA, Harsha D, et al. Effects on blood pressure of reduced dietary sodium and the Dietary Approaches to Stop Hypertension (DASH) diet. DASH-Sodium Collaborative Research Group. N Engl J Med 2001;344:310.

178. Zhang Z, Xu G, Liu D, Zhu W, Fan X, Liu X. Dietary fiber consumption and risk of stroke. Eur J Epidemiol 2013;28:119-30.

179. Threapleton DE, Greenwood DC, Evans CE, Cleghorn CL, Nykjaer C, Woodhead C, et al. Dietary fibre intake and risk of cardiovascular disease: systematic review and meta-analysis. BMJ 2013;347:f6879. 


\section{APPENDIX}

*The following process was used in the literature search and review for each key question:

1. Searching Strategies

2. Flow Chart of the Study Selection Process

3. Finally Included Studies

4. Evidence Table for Assessment of Risk of Bias and Quality

\section{INTRODUCTION OF CARDIAC REHABILITATION}

The following are the basic searching strategies used for all key questions of the introduction part in the Cochrane Library. These basic searching strategies were combined with the specific searching strategy for each question using AND.

\#1 MeSH descriptor: [Cardiac Rehabilitation] explode all trees

\#2 ((*ardia* or heart*) and *habilitation*):ti,ab,kw

\#3 \#1 or \#2

\#4 MeSH descriptor: [Myocardial Ischemia] explode all trees

\#5 ((myocard* ${ }^{*}$ or heart* or coronary or cardia*) and (infarct* or isch* or attack*)):ti,ab,kw

\#6 ("acute coronary syndrome" or ACS):ti,ab,kw

\#7 (angina or stenocardia*):ti,ab,kw

\#8 MeSH descriptor: [Myocardial Revascularization] explode all trees

\#9 ((Myocard* or cardi* or coronary) and (Revascular* or angioplast*)):ti,ab,kw

\#10 ((coronary or rotational) near atherectom*):ti,ab,kw

\#11 ("coronary artery bypass" or CABG or "aortocoronary bypass" or "coronary bypass"):ti,ab,kw

\#12 MeSH descriptor: [Percutaneous Coronary Intervention] explode all trees

\#13 ("percutaneous coronary intervention*" or PCI):ti,ab,kw

\#14 (percutaneous next coronary near/2 (interven* or revascular*)):ti,ab,kw

\#15 (stent* and (heart or cardiac*)):ti,ab,kw

\#16 coronary near (disease* or bypass or thrombo* or angioplast*):ti,ab,kw

\#17 (PTCA or "percutaneous transluminal coronary angioplasty"):ti,ab,kw

\#18 MeSH descriptor: [Heart Bypass, Right] explode all trees

\#19 ("heart manual"):ti,ab,kw

\#20 (arrhythmia* or dysrhythmia* or bradycardia* or tachycardia*):ti,ab,kw

\#21 (cardiopulmonary next arrest* or cardio-pulmonary next arrest*):ti,ab,kw

\#22 heart failure:ti,ab,kw

\#23 ((heart or cardiac or coronary) near/3 transplant*):ti,ab,kw

\#24 \{or \#4-\#23\}

\#25 (arthritis* or cancer* or stroke* or kidney* or "obstructive pulmonary" or claudication* or fracture* or Parkinson*):ti,ab,kw

\#26 \#24 not \#25

\#27 MeSH descriptor: [Rehabilitation] explode all trees

\#28 (rehabilitat* or *habilitation):ti,ab,kw

\#29 MeSH descriptor: [Physical and Rehabilitation Medicine] explode all trees

\#30 MeSH descriptor: [Health Facilities] explode all trees

\#31 MeSH descriptor: [Rehabilitation Nursing] explode all trees 


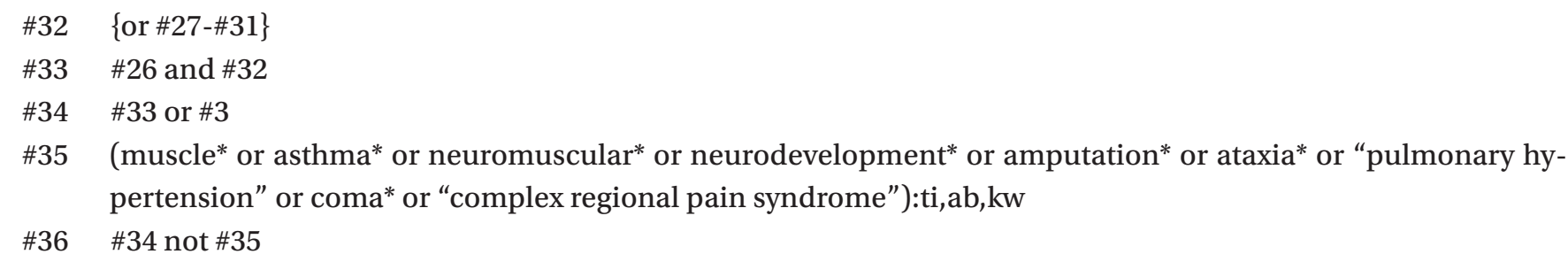

The same Cochrane Library searching strategy was used for KQ1 ("Should cardiac rehabilitation be an integral component of the care of acute coronary syndrome?") and KQ4 ("When should cardiac rehabilitation exercise begin?"). The following is the final searching strategy, including the basic CR searching strategy:

\section{Searching Strategies}

\#37 MeSH descriptor: [Activities of Daily Living] explode all trees

\#38 MeSH descriptor: [Exercise] explode all trees

\#39 MeSH descriptor: [Exercise Therapy] explode all trees

\#40 (exercise* near/2 (rehabilitat* or therap* or training or program* or activit* or toleran* or prescribe* or prescription* or structure* or unstructure* or un-structure* or supervise* or unsupervise* or un-supervise* or guided or unguided or dynamic or regime*)):ti,ab,kw

\#41 (physical near/2 (exercise* or educat* or training or program* or activit* or regime*)):ti,ab,kw

\#42 (aerobic* near/2 (exercise* or training or program* or activit* or regime*)):ti,ab,kw

\#43 (strength* near (exercise* or training)):ti, ab,kw

\#44 (endurance near (exercise* or training)):ti,ab,kw

\#45 (fitness near/2 (training or program* or regime*)):ti,ab,kw

\#46 ((resistance or resistive) near (exercise* or training)):ti, ab,kw

\#47 (isometric near/2 (exercise* or training or program* or activit* or regime*)):ti,ab,kw

\#48 ((high* frequency or low* frequency) near/2 (exercise* or training or program* or activit* or regime*)):ti,ab,kw

\#49 ((high* intensi* ${ }^{*}$ or low* intensi*) near/2 (exercise* or training or program* or activit* or regime*)):ti,ab,kw

\#50 MeSH descriptor: [Managed Care Programs] explode all trees

\#51 ((multifactor* or multifacet* or managed care) near program*):ti,ab,kw

\#52 \{or \#37-\#51\}

\#53 \#36 and \#52 Publication Year from 2009 to 2018 
Flow Chart of Study Selection Process

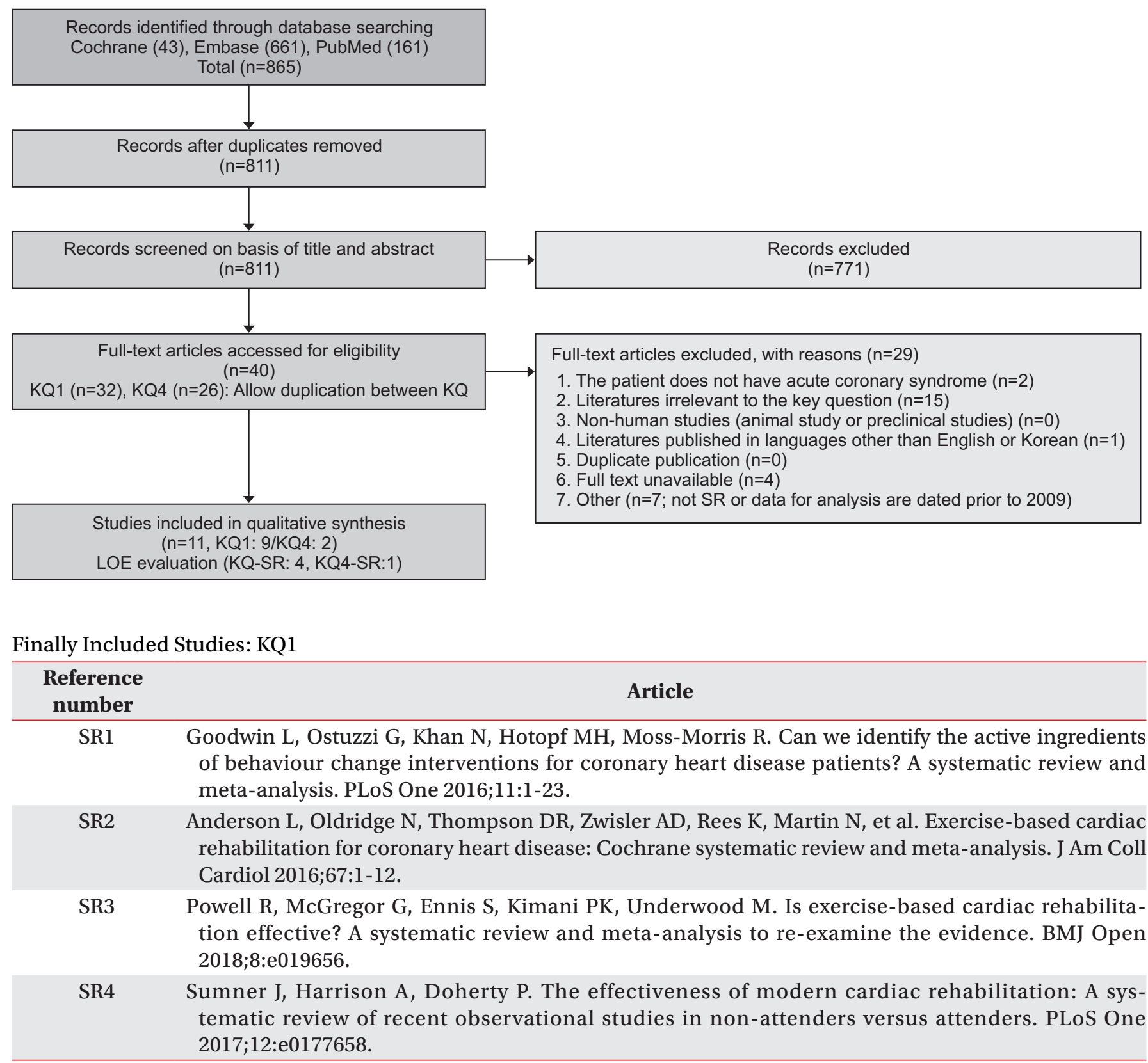

SR, systematic reviews. 
Evidence Table for Assessment of Methodological Quality of Systematic Reviews using AMSTAR 2 and Level of Evidence (LOE) using SIGN Methods: KQ1

\begin{tabular}{|c|c|c|c|c|c|c|c|c|c|c|c|c|c|c|c|c|c|}
\hline \multirow{2}{*}{$\begin{array}{c}\text { Reference } \\
\text { number }\end{array}$} & \multicolumn{16}{|c|}{ Quality items } & \multirow{2}{*}{ LOI } \\
\hline & 1 & 2 & 3 & 4 & 5 & 6 & 7 & 8 & 9 & 10 & 11 & 12 & 13 & 14 & 15 & 16 & \\
\hline SR1 & $\mathrm{Y}$ & $\mathrm{P}$ & $\mathrm{Y}$ & $\mathrm{P}$ & $\mathrm{Y}$ & $\mathrm{Y}$ & $\mathrm{Y}$ & $\mathrm{Y}$ & $\mathrm{Y}$ & $\mathrm{N}$ & $\mathrm{Y}$ & $\mathrm{Y}$ & $\mathrm{Y}$ & $\mathrm{Y}$ & $\mathrm{Y}$ & $\mathrm{Y}$ & $1++$ \\
\hline SR2 & $\mathrm{Y}$ & $\mathrm{N}$ & $\mathrm{Y}$ & $\mathrm{N}$ & $\mathrm{Y}$ & $\mathrm{Y}$ & $\mathrm{Y}$ & $\mathrm{P}$ & $\mathrm{Y}$ & $\mathrm{N}$ & $\mathrm{Y}$ & $\mathrm{Y}$ & $\mathrm{Y}$ & $\mathrm{Y}$ & $\mathrm{Y}$ & $\mathrm{Y}$ & $1++$ \\
\hline SR3 & $\mathrm{Y}$ & $\mathrm{P}$ & $\mathrm{Y}$ & $\mathrm{N}$ & $\mathrm{Y}$ & $\mathrm{N}$ & $\mathrm{Y}$ & $\mathrm{Y}$ & $\mathrm{Y}$ & $\mathrm{N}$ & $\mathrm{Y}$ & $\mathrm{Y}$ & $\mathrm{Y}$ & $\mathrm{Y}$ & $\mathrm{N}$ & $\mathrm{Y}$ & $1++$ \\
\hline SR4 & $\mathrm{Y}$ & $\mathrm{N}$ & $\mathrm{N}$ & $\mathrm{P}$ & $\mathrm{Y}$ & $\mathrm{Y}$ & $\mathrm{Y}$ & $\mathrm{P}$ & $\mathrm{Y}$ & $\mathrm{N}$ & $\mathrm{Y}$ & $\mathrm{Y}$ & $\mathrm{Y}$ & $\mathrm{Y}$ & $\mathrm{N}$ & $\mathrm{Y}$ & $1++$ \\
\hline
\end{tabular}

1. Did the research questions and inclusion criteria for the review include the components of PICO? 2. Did the report of the review contain an explicit statement that the review methods were established prior to the conduct of the review and did the report justify any significant deviations from the protocol? 3. Did the review authors explain their selection of the study designs for inclusion in the review? 4. Did the review authors use a comprehensive literature search strategy? 5. Did the review authors perform study selection in duplicate? 6. Did the review authors perform data extraction in duplicate? 7. Did the review authors provide a list of excluded studies and justify the exclusions? 8. Did the review authors describe the included studies in adequate detail? 9. Did the review authors use a satisfactory technique for assessing the risk of bias (RoB) in individual studies that were included in the review? 10. Did the review authors report on the sources of funding for the studies included in the review? 11. If meta-analysis was performed did the review authors use appropriate methods for statistical combination of results? 12. If meta-analysis was performed, did the review authors assess the potential impact of RoB in individual studies on the results of the meta-analysis or other evidence synthesis? 13. Did the review authors account for RoB in individual studies when interpreting/ discussing the results of the review? 14. Did the review authors provide a satisfactory explanation for, and discussion of, any heterogeneity observed in the results of the review? 15. If they performed quantitative synthesis did the review authors carry out an adequate investigation of publication bias (small study bias) and discuss its likely impact on the results of the review? 16. Did the review authors report any potential sources of conflict of interest, including any funding they received for conducting the review?

Y, yes; N, no; P, partial yes.

Finally Included Studies: KQ4

\begin{tabular}{cc}
$\begin{array}{c}\text { Reference } \\
\text { number }\end{array}$ & Article \\
\hline SR1 & $\begin{array}{c}\text { Zhang YM, Lu Y, Tang Y, Yang D, Wu HF, Bian ZP, et al. The effects of different initiation time of exercise } \\
\text { training on left ventricular remodeling and cardiopulmonary rehabilitation in patients with left ventric- } \\
\text { ular dysfunction after myocardial infarction. Disabil Rehabil 2016;38:268-76. }\end{array}$ \\
& ular \\
\hline
\end{tabular}

SR, systematic reviews. 
Evidence Table for Assessment of Methodological Quality of Systematic Reviews using AMSTAR 2.0 and Level of Evidence (LOE) using SIGN Methods: KQ4

\begin{tabular}{ccccccccccccccccccc}
\hline $\begin{array}{c}\text { Reference } \\
\text { number }\end{array}$ & $\mathbf{1}$ & $\mathbf{2}$ & $\mathbf{3}$ & $\mathbf{4}$ & $\mathbf{5}$ & $\mathbf{6}$ & $\mathbf{7}$ & $\mathbf{8}$ & $\mathbf{9}$ & $\mathbf{1 0}$ & $\mathbf{1 1}$ & $\mathbf{1 2}$ & $\mathbf{1 3}$ & $\mathbf{1 4}$ & $\mathbf{1 5}$ & $\mathbf{1 6}$ & \multirow{2}{*}{ LOE } \\
\hline $\mathrm{S}$ & $\mathrm{Y}$ & $\mathrm{P}$ & $\mathrm{Y}$ & $\mathrm{P}$ & $\mathrm{Y}$ & $\mathrm{Y}$ & $\mathrm{Y}$ & $\mathrm{P}$ & $\mathrm{Y}$ & $\mathrm{N}$ & $\mathrm{Y}$ & $\mathrm{Y}$ & $\mathrm{Y}$ & $\mathrm{Y}$ & $\mathrm{Y}$ & $\mathrm{Y}$ & $1++$ \\
\hline
\end{tabular}

1. Did the research questions and inclusion criteria for the review include the components of PICO? 2. Did the report of the review contain an explicit statement that the review methods were established prior to the conduct of the review and did the report justify any significant deviations from the protocol? 3. Did the review authors explain their selection of the study designs for inclusion in the review? 4. Did the review authors use a comprehensive literature search strategy? 5. Did the review authors perform study selection in duplicate? 6. Did the review authors perform data extraction in duplicate? 7. Did the review authors provide a list of excluded studies and justify the exclusions? 8 . Did the review authors describe the included studies in adequate detail? 9. Did the review authors use a satisfactory technique for assessing the risk of bias (RoB) in individual studies that were included in the review? 10. Did the review authors report on the sources of funding for the studies included in the review? 11. If meta-analysis was performed did the review authors use appropriate methods for statistical combination of results? 12. If meta-analysis was performed, did the review authors assess the potential impact of RoB in individual studies on the results of the meta-analysis or other evidence synthesis? 13. Did the review authors account for RoB in individual studies when interpreting/ discussing the results of the review? 14. Did the review authors provide a satisfactory explanation for, and discussion of, any heterogeneity observed in the results of the review? 15. If they performed quantitative synthesis did the review authors carry out an adequate investigation of publication bias (small study bias) and discuss its likely impact on the results of the review? 16. Did the review authors report any potential sources of conflict of interest, including any funding they received for conducting the review?

$\mathrm{Y}$, yes; N, no; P, partial yes.

The following is the final Cochrane Library searching strategy used for KQ2 ("Does cardiac rehabilitation affect the outcomes of patients with cardiovascular disease?") including the basic CR searching strategy:

\section{Searching Strategies}

\#37 ("Cardiovascular mortality" or "all-cause mortality" or "hospital readmission rates" or morbidity or "Quality of life" or QOL or "mental health" or "return to work"):ti,ab,kw

\#38 MeSH descriptor: [Morbidity] explode all trees

\#39 MeSH descriptor: [Quality of Life] explode all trees

\#40 MeSH descriptor: [Mental Health] explode all trees

\#41 MeSH descriptor: [Return to Work] explode all trees

\#42 self-efficacy or "patient satisfaction" or "CV recurrence rate" or "incidence of recurrent" or "fatal MI" or "nonfatal MI"

\#43 incidence of recurrent MI or "progression of coronary atherosclerosis" or depression*

\#44 \{or \#37-\#43\}

\#45 \#36 and \#44 Publication Year from 2017 to 2018 


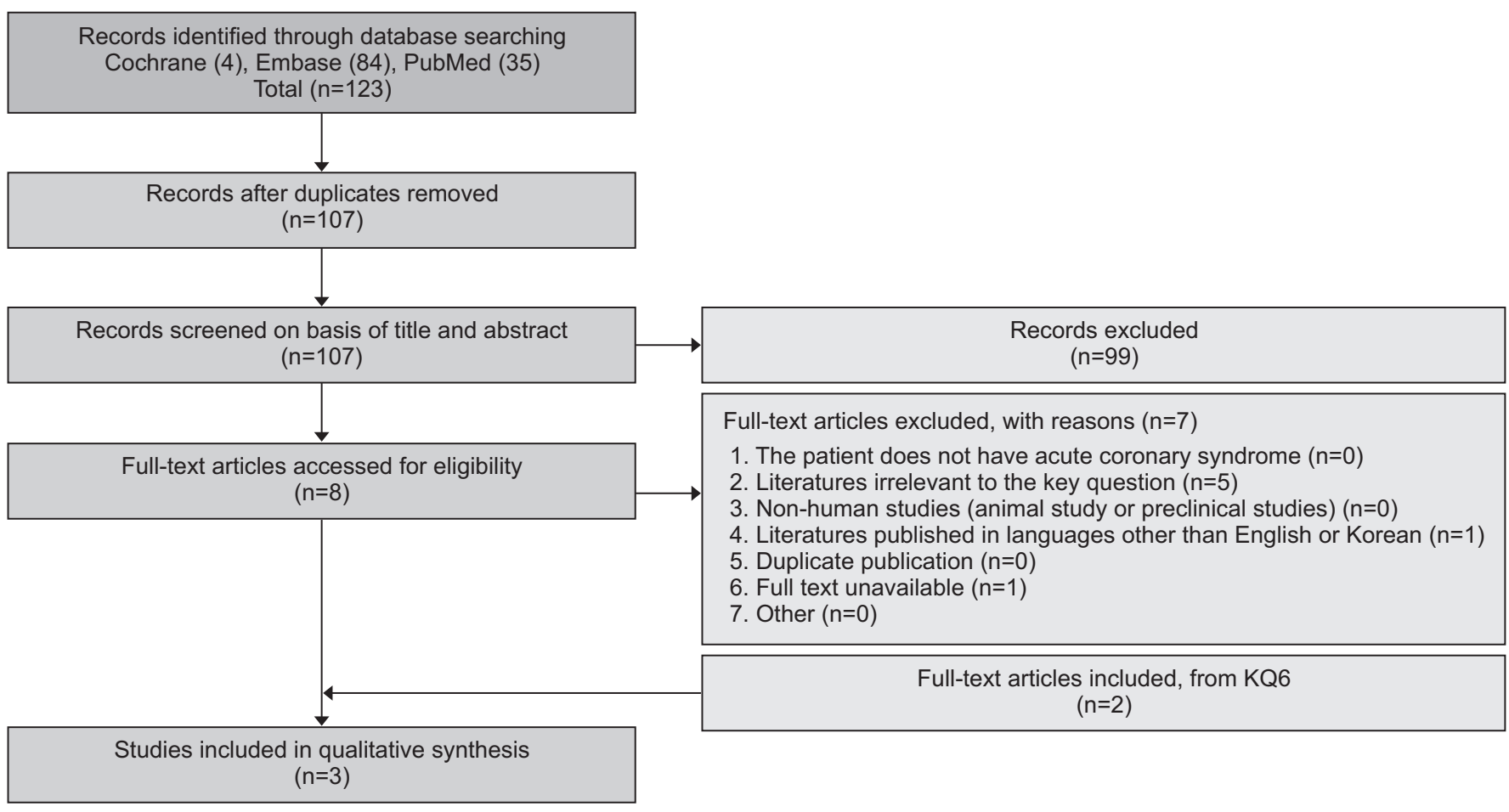

Finally Included Studies

\begin{tabular}{cc}
$\begin{array}{c}\text { Reference } \\
\text { number }\end{array}$ & Article \\
\hline SR1 & $\begin{array}{c}\text { Abell B, Glasziou P, Hoffmann T. The contribution of individual exercise training components to clini- } \\
\text { cal outcomes in randomised controlled trials of cardiac rehabilitation: a systematic review and meta- } \\
\text { regression. Sports Med Open 2017;3(1):19. }\end{array}$ \\
SR2 & $\begin{array}{c}\text { van Halewijn G, Deckers J, Tay HY, van Domburg R, Kotseva K, Wood D. Lessons from contemporary } \\
\text { trials of cardiovascular prevention and rehabilitation: a systematic review and meta-analysis. Int J Car- } \\
\text { diol 201;232:294-303. }\end{array}$ \\
SR3 & $\begin{array}{c}\text { Anderson L, Oldridge N, Thompson DR, Zwisler AD, Rees K, Martin N, et al. Exercise-based cardiac re- } \\
\text { habilitation for coronary heart disease: Cochrane systematic review and meta-analysis. J Am Coll Car- } \\
\text { diol 2016;67:1-12. }\end{array}$ \\
\hline
\end{tabular}

SR, systematic reviews. 
Evidence Table for Assessment of Methodological Quality of Systematic Reviews using AMSTAR 2.0 and Level of Evidence (LOE) using SIGN Methods: KQ 2

\begin{tabular}{ccccccccccccccccccc}
\hline $\begin{array}{c}\text { Reference } \\
\text { number }\end{array}$ & $\mathbf{1}$ & $\mathbf{2}$ & $\mathbf{3}$ & $\mathbf{4}$ & $\mathbf{5}$ & $\mathbf{6}$ & $\mathbf{7}$ & $\mathbf{8}$ & $\mathbf{9}$ & $\mathbf{1 0}$ & $\mathbf{1 1}$ & $\mathbf{1 2}$ & $\mathbf{1 3}$ & $\mathbf{1 4}$ & $\mathbf{1 5}$ & $\mathbf{1 6}$ & \multirow{2}{*}{ LOE } \\
\hline SR1 & $\mathrm{Y}$ & $\mathrm{Y}$ & $\mathrm{Y}$ & $\mathrm{Y}$ & $\mathrm{Y}$ & $\mathrm{N}$ & $\mathrm{N}$ & $\mathrm{Y}$ & $\mathrm{P}$ & $\mathrm{N}$ & $\mathrm{Y}$ & $\mathrm{Y}$ & $\mathrm{Y}$ & $\mathrm{Y}$ & $\mathrm{Y}$ & $\mathrm{Y}$ & $1++$ \\
SR2 & $\mathrm{Y}$ & $\mathrm{P}$ & $\mathrm{Y}$ & $\mathrm{P}$ & $\mathrm{Y}$ & $\mathrm{Y}$ & $\mathrm{N}$ & $\mathrm{N}$ & $\mathrm{P}$ & $\mathrm{N}$ & $\mathrm{Y}$ & $\mathrm{Y}$ & $\mathrm{Y}$ & $\mathrm{Y}$ & $\mathrm{Y}$ & $\mathrm{Y}$ & $1++$ \\
SR3 & $\mathrm{Y}$ & $\mathrm{N}$ & $\mathrm{Y}$ & $\mathrm{N}$ & $\mathrm{Y}$ & $\mathrm{Y}$ & $\mathrm{Y}$ & $\mathrm{P}$ & $\mathrm{Y}$ & $\mathrm{N}$ & $\mathrm{Y}$ & $\mathrm{Y}$ & $\mathrm{Y}$ & $\mathrm{Y}$ & $\mathrm{Y}$ & $\mathrm{Y}$ & $1++$ \\
\hline
\end{tabular}

1. Did the research questions and inclusion criteria for the review include the components of PICO? 2. Did the report of the review contain an explicit statement that the review methods were established prior to the conduct of the review and did the report justify any significant deviations from the protocol? 3. Did the review authors explain their selection of the study designs for inclusion in the review? 4. Did the review authors use a comprehensive literature search strategy? 5. Did the review authors perform study selection in duplicate? 6. Did the review authors perform data extraction in duplicate? 7. Did the review authors provide a list of excluded studies and justify the exclusions? 8 . Did the review authors describe the included studies in adequate detail? 9. Did the review authors use a satisfactory technique for assessing the risk of bias (RoB) in individual studies that were included in the review? 10. Did the review authors report on the sources of funding for the studies included in the review? 11. If meta-analysis was performed did the review authors use appropriate methods for statistical combination of results? 12. If meta-analysis was performed, did the review authors assess the potential impact of RoB in individual studies on the results of the meta-analysis or other evidence synthesis? 13. Did the review authors account for RoB in individual studies when interpreting/ discussing the results of the review? 14. Did the review authors provide a satisfactory explanation for, and discussion of, any heterogeneity observed in the results of the review? 15. If they performed quantitative synthesis did the review authors carry out an adequate investigation of publication bias (small study bias) and discuss its likely impact on the results of the review? 16. Did the review authors report any potential sources of conflict of interest, including any funding they received for conducting the review?

Y, yes; N, no; P, partial yes.

The following is the final Cochrane Library searching strategy used for KQ3 ("Does cardiac rehabilitation improve the quality of life of patients with cardiovascular disease?”) including the basic CR searching strategy:

\section{Searching Strategies}

\#37 ("quality of life" or qol):ti,ab,kw

\#38 ("quality of wellbeing" or "quality of well being" or qwb):ti,ab,kw

\#39 MeSH descriptor: [Quality of Life] explode all trees

\#40 \{or \#37-\#39\}

\#41 \#36 and \#40 Publication Year from 2017 to 2018 
Chul Kim, et al.

Flow Chart of Study Selection Process

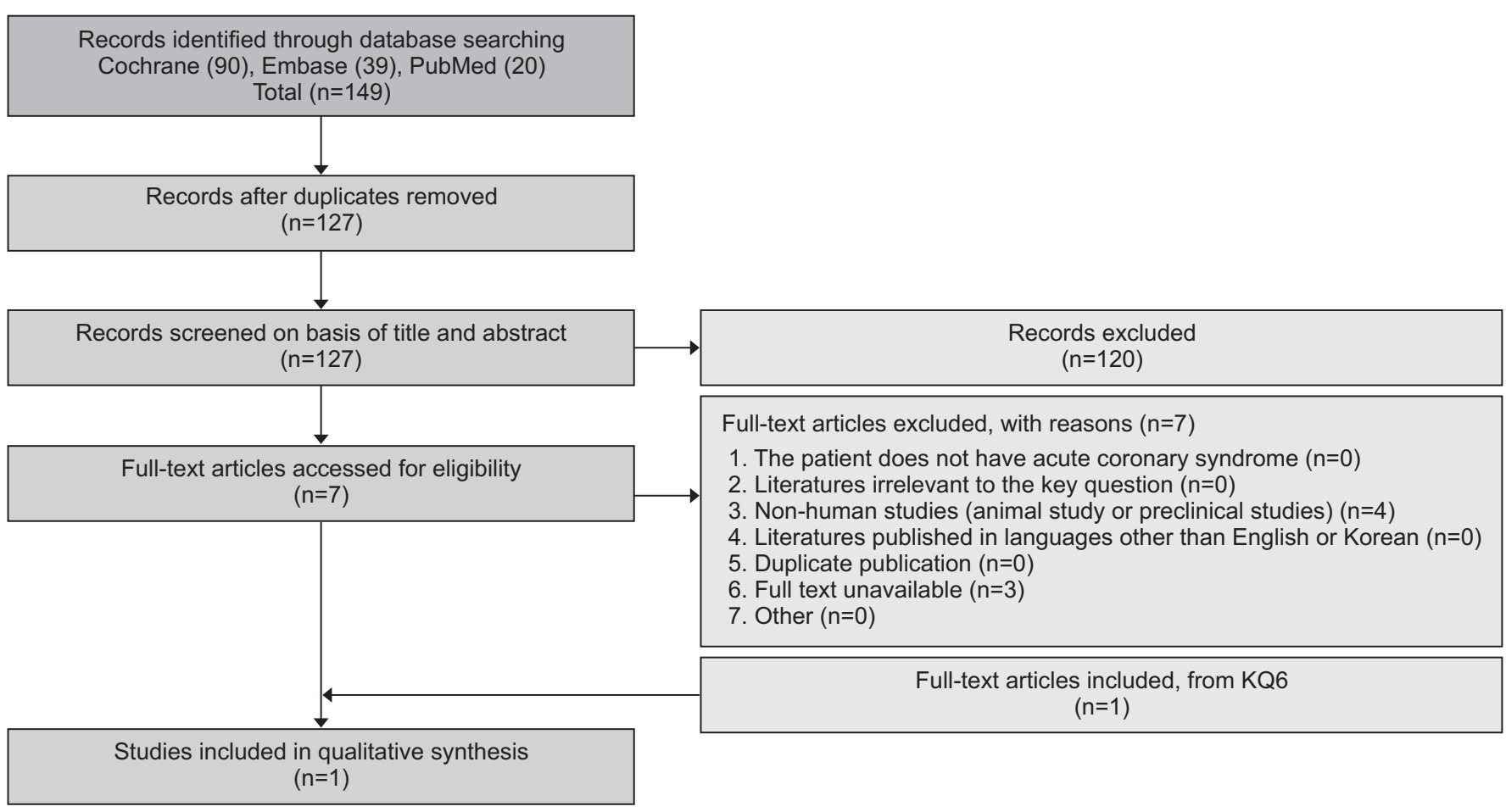

Finally Included Studies

\begin{tabular}{cc}
$\begin{array}{c}\text { Reference } \\
\text { number }\end{array}$ & Article \\
\hline SR1 & $\begin{array}{c}\text { Anderson L, Oldridge N, Thompson DR, et al. Exercise-Based Cardiac Rehabilitation for Coronary Heart } \\
\text { Disease Cochrane Systematic Review and Meta-Analysis. J Am Coll Cardiol 2016;67(1):1-12. }\end{array}$ \\
\hline
\end{tabular}

SR, systematic reviews. 
Evidence Table for Assessment of Methodological Quality of Systematic Reviews using AMSTAR 2.0 and Level of Evidence (LOE) using SIGN Methods:

\begin{tabular}{ccccccccccccccccccc}
\hline $\begin{array}{c}\text { Reference } \\
\text { number }\end{array}$ & $\mathbf{1}$ & $\mathbf{2}$ & $\mathbf{3}$ & $\mathbf{4}$ & $\mathbf{5}$ & $\mathbf{6}$ & $\mathbf{7}$ & $\mathbf{8}$ & $\mathbf{9}$ & $\mathbf{1 0}$ & $\mathbf{1 1}$ & $\mathbf{1 2}$ & $\mathbf{1 3}$ & $\mathbf{1 4}$ & $\mathbf{1 5}$ & $\mathbf{1 6}$ & \multirow{2}{*}{ LOE } \\
\hline SR1 & $\mathrm{Y}$ & $\mathrm{N}$ & $\mathrm{Y}$ & $\mathrm{N}$ & $\mathrm{Y}$ & $\mathrm{Y}$ & $\mathrm{Y}$ & $\mathrm{P}$ & $\mathrm{Y}$ & $\mathrm{N}$ & $\mathrm{Y}$ & $\mathrm{Y}$ & $\mathrm{Y}$ & $\mathrm{Y}$ & $\mathrm{Y}$ & $\mathrm{Y}$ & $1++$ \\
\hline
\end{tabular}

1. Did the research questions and inclusion criteria for the review include the components of PICO? 2. Did the report of the review contain an explicit statement that the review methods were established prior to the conduct of the review and did the report justify any significant deviations from the protocol? 3. Did the review authors explain their selection of the study designs for inclusion in the review? 4. Did the review authors use a comprehensive literature search strategy? 5. Did the review authors perform study selection in duplicate? 6. Did the review authors perform data extraction in duplicate? 7. Did the review authors provide a list of excluded studies and justify the exclusions? 8. Did the review authors describe the included studies in adequate detail? 9. Did the review authors use a satisfactory technique for assessing the risk of bias (RoB) in individual studies that were included in the review? 10. Did the review authors report on the sources of funding for the studies included in the review? 11. If meta-analysis was performed did the review authors use appropriate methods for statistical combination of results? 12. If meta-analysis was performed, did the review authors assess the potential impact of RoB in individual studies on the results of the meta-analysis or other evidence synthesis? 13. Did the review authors account for RoB in individual studies when interpreting/ discussing the results of the review? 14. Did the review authors provide a satisfactory explanation for, and discussion of, any heterogeneity observed in the results of the review? 15. If they performed quantitative synthesis did the review authors carry out an adequate investigation of publication bias (small study bias) and discuss its likely impact on the results of the review? 16. Did the review authors report any potential sources of conflict of interest, including any funding they received for conducting the review?

Y, yes; N, no; P, partial yes.

The following is the final Cochrane Library searching strategy used for KQ5 ("How should cardiac rehabilitation be structured?") including the basic CR searching strategy:

\section{Searching Strategies}

\#37 (process* or facilit* or machine* or treadmill* or "cycle ergometer*" or "ECG monitor*" or "blood pressure monitor*" or "automated external defibrillator" or AED or "emergency cart*" or "12-lead ECG" or "blood glucose monitor*" or "percutaneous oxygen saturation monitor*" or "spirometry device*"):ti,ab,kw

\#38 (cardiology* or dietetic* or nursing* or "exercise physiolog*" or "occupational therap*" or physiotherap* or psycholog* or "social work"):ti,ab,kw

\#39 ("organizational structure" or policy or polices or process* or construction* or concept* or content* or phase* or "flow chart" or "aerobic exercise machine*" or treadmill or cycle* or ergometer* or "medical director*" or director* or nurse* or "exercise physiologist*" or "occupational therapist*" or physiotherapist* or psychologist* or "social work*" or dietician*):ti,ab,kw

\#40 (Facility or Equipment or Personnel* or medical staff* or staff* or Service* or "service framework*" or Phase*):ti,ab,kw

\#41 MeSH descriptor: [Organizations] explode all trees

\#42 MeSH descriptor: [Delivery of Health Care] explode all trees

\#43 \{or \#37-\#42\}

\#44 \#36 and \#43 Publication Year from 2012 to 2018 


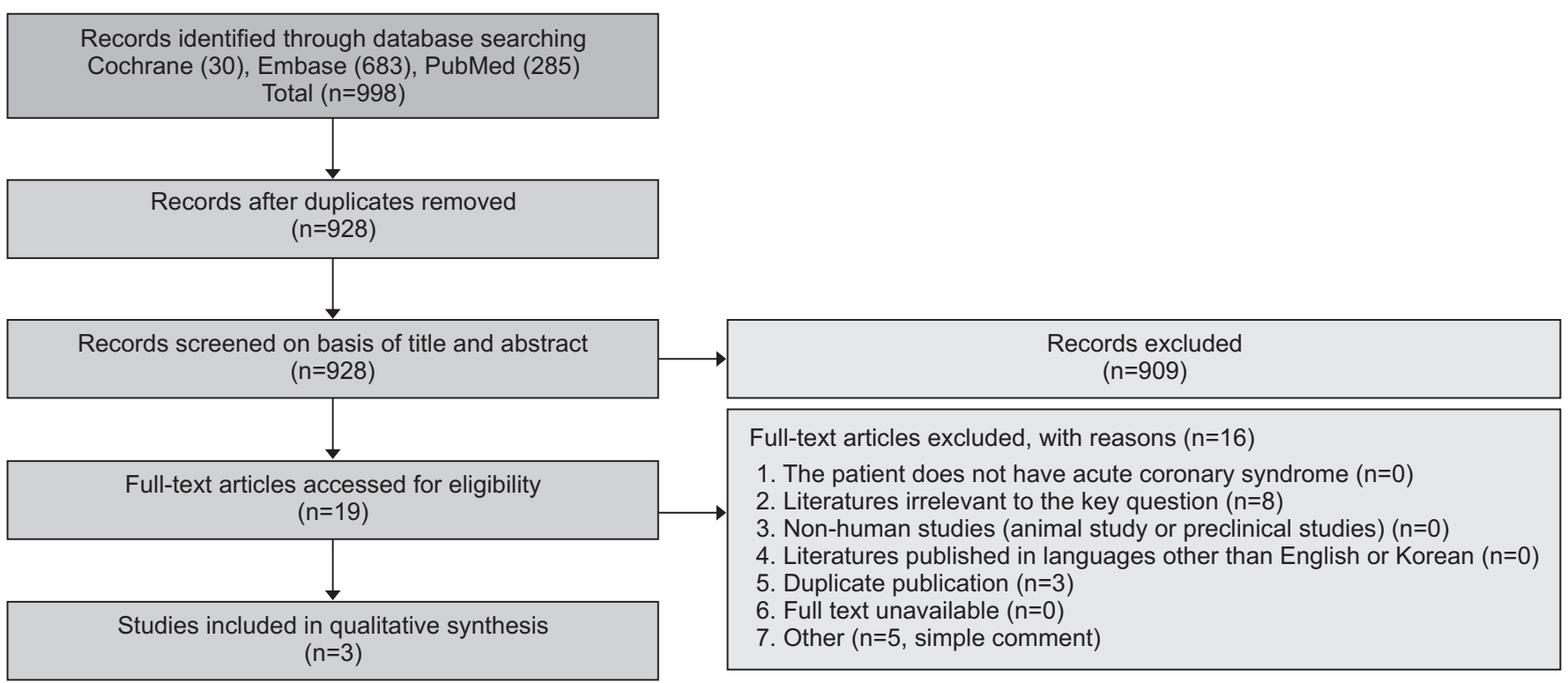

Finally Included Studies

\begin{tabular}{cc}
\hline $\begin{array}{c}\text { Reference } \\
\text { number }\end{array}$ & Article \\
\hline SR1 & $\begin{array}{c}\text { Anderson L, Oldridge N, Thompson DR, Zwisler AD, Rees K, Martin N, et al. Exercise-based cardiac re- } \\
\text { habilitation for coronary heart disease: Cochrane systematic review and meta-analysis. J Am Coll Car- } \\
\text { diol 2016;67:1-12. }\end{array}$ \\
SR2 & $\begin{array}{c}\text { Sumner J, Harrison A, Doherty P. The effectiveness of modern cardiac rehabilitation: A systematic re- } \\
\text { view of recent observational studies in non-attenders versus attenders. PLoS One 2017;12:e0177658. } \\
\text { SR3 } \\
\text { Oldridge N. Exercise-based cardiac rehabilitation in patients with coronary heart disease: meta-analysis } \\
\text { outcomes revisited. Future Cardiol 2012;8:729-51. }\end{array}$ \\
\hline
\end{tabular}

SR, systematic reviews. 
Evidence Table for Assessment of Methodological Quality of Systematic Reviews using AMSTAR 2.0 and Level of Evidence (LOE) using SIGN Methods: KQ 5

\begin{tabular}{ccccccccccccccccccc}
\hline $\begin{array}{c}\text { Reference } \\
\text { number }\end{array}$ & $\mathbf{1}$ & $\mathbf{2}$ & $\mathbf{3}$ & $\mathbf{4}$ & $\mathbf{5}$ & $\mathbf{6}$ & $\mathbf{7}$ & $\mathbf{8}$ & $\mathbf{9}$ & $\mathbf{1 0}$ & $\mathbf{1 1}$ & $\mathbf{1 2}$ & $\mathbf{1 3}$ & $\mathbf{1 4}$ & $\mathbf{1 5}$ & $\mathbf{1 6}$ & \multirow{2}{*}{ LOE } \\
\hline SR1 & $\mathrm{Y}$ & $\mathrm{N}$ & $\mathrm{Y}$ & $\mathrm{N}$ & $\mathrm{Y}$ & $\mathrm{Y}$ & $\mathrm{Y}$ & $\mathrm{P}$ & $\mathrm{Y}$ & $\mathrm{N}$ & $\mathrm{Y}$ & $\mathrm{Y}$ & $\mathrm{Y}$ & $\mathrm{Y}$ & $\mathrm{Y}$ & $\mathrm{Y}$ & $1++$ \\
SR2 & $\mathrm{Y}$ & $\mathrm{N}$ & $\mathrm{N}$ & $\mathrm{P}$ & $\mathrm{Y}$ & $\mathrm{Y}$ & $\mathrm{Y}$ & $\mathrm{P}$ & $\mathrm{Y}$ & $\mathrm{N}$ & $\mathrm{Y}$ & $\mathrm{Y}$ & $\mathrm{Y}$ & $\mathrm{Y}$ & $\mathrm{N}$ & $\mathrm{Y}$ & $1++$ \\
SR3 & $\mathrm{Y}$ & $\mathrm{P}$ & $\mathrm{Y}$ & $\mathrm{P}$ & $\mathrm{N}$ & $\mathrm{N}$ & $\mathrm{N}$ & $\mathrm{P}$ & $\mathrm{Y}$ & $\mathrm{Y}$ & $\mathrm{Y}$ & $\mathrm{Y}$ & $\mathrm{Y}$ & $\mathrm{Y}$ & $\mathrm{N}$ & $\mathrm{Y}$ & $1++$ \\
\hline
\end{tabular}

1. Did the research questions and inclusion criteria for the review include the components of PICO? 2. Did the report of the review contain an explicit statement that the review methods were established prior to the conduct of the review and did the report justify any significant deviations from the protocol? 3. Did the review authors explain their selection of the study designs for inclusion in the review? 4. Did the review authors use a comprehensive literature search strategy? 5. Did the review authors perform study selection in duplicate? 6. Did the review authors perform data extraction in duplicate? 7. Did the review authors provide a list of excluded studies and justify the exclusions? 8 . Did the review authors describe the included studies in adequate detail? 9. Did the review authors use a satisfactory technique for assessing the risk of bias (RoB) in individual studies that were included in the review? 10. Did the review authors report on the sources of funding for the studies included in the review? 11. If meta-analysis was performed did the review authors use appropriate methods for statistical combination of results? 12. If meta-analysis was performed, did the review authors assess the potential impact of RoB in individual studies on the results of the meta-analysis or other evidence synthesis? 13. Did the review authors account for RoB in individual studies when interpreting/ discussing the results of the review? 14. Did the review authors provide a satisfactory explanation for, and discussion of, any heterogeneity observed in the results of the review? 15. If they performed quantitative synthesis did the review authors carry out an adequate investigation of publication bias (small study bias) and discuss its likely impact on the results of the review? 16. Did the review authors report any potential sources of conflict of interest, including any funding they received for conducting the review?

$\mathrm{Y}=\mathrm{yes}, \mathrm{N}=$ no, $\mathrm{P}=$ partial yes.

The following is the final Cochrane Library searching strategy used for KQ6 ("Can cardiac rehabilitation programs lower the cost of health management for patients with acute coronary syndrome?") including the basic CR searching strategy:

\section{Searching Strategies}

\#37 MeSH descriptor: [Costs and Cost Analysis] explode all trees

\#38 MeSH descriptor: [Economics] explode all trees

\#39 MeSH descriptor: [Fees and Charges] explode all trees

\#40 MeSH descriptor: [Budgets] explode all trees

\#41 (cost* near/2 (effective* or utilit* or benefit* or minimi*)):ti,ab,kw

\#42 (price or pricing or financ* or fee or fees):ti, ab,kw

\#43 (value near/2 (money or monetary)):ti,ab,kw

\#44 \{or \#37-\#43\}

\#45 \#36 and \#44 


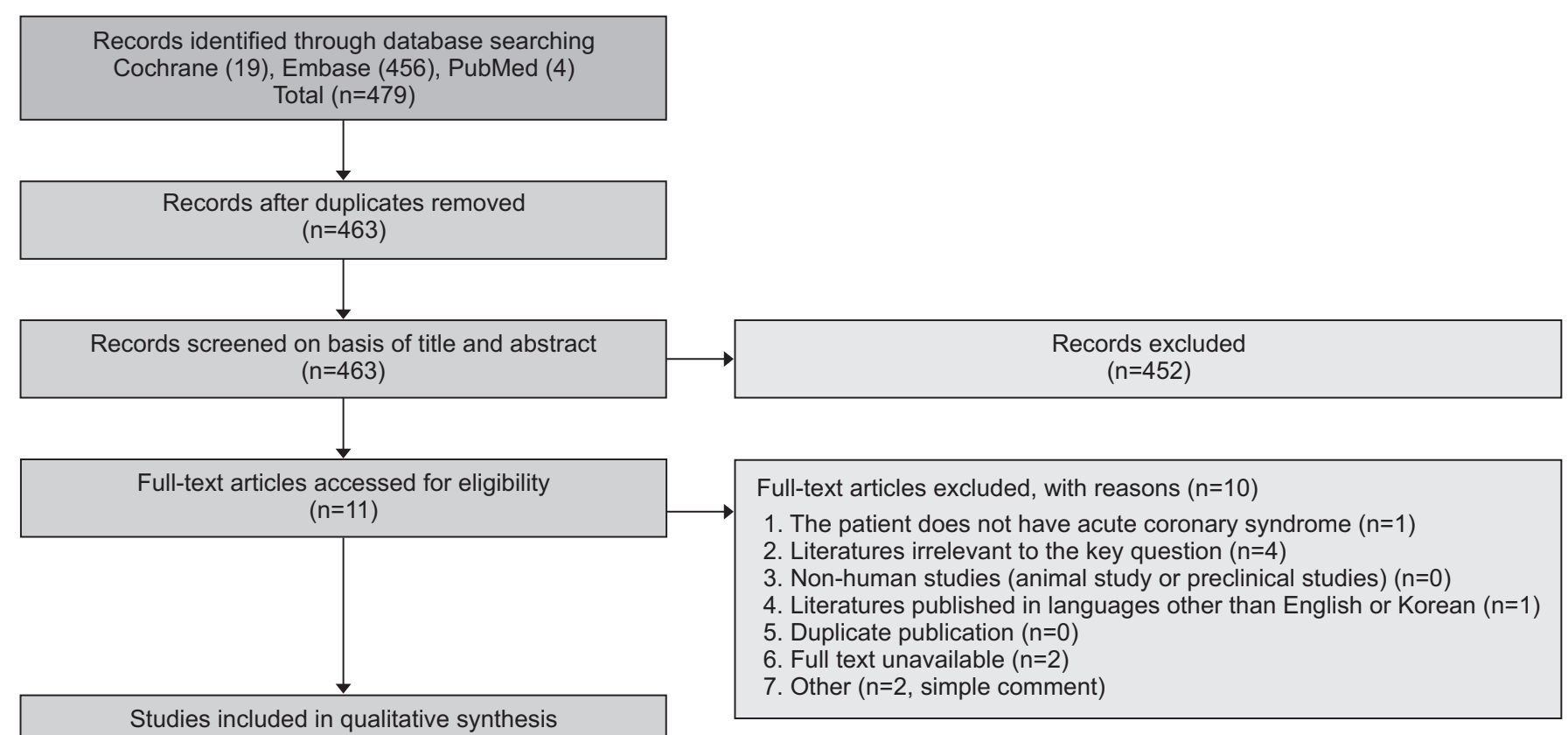

Finally Included Studies

\begin{tabular}{cl}
$\begin{array}{c}\text { Reference } \\
\text { number }\end{array}$ & Article \\
\hline SR1 & $\begin{array}{c}\text { Anderson L, Oldridge N, Thompson DR, Zwisler AD, Rees K, Martin N, et al. Exercise-based cardiac reha- } \\
\text { bilitation for coronary heart disease: Cochrane systematic review and meta-analysis. J Am Coll Cardiol } \\
\end{array}$ \\
& $2016 ; 67: 1-12$.
\end{tabular}

SR, systematic reviews. 
Evidence Table for Assessment of Methodological Quality of Systematic Reviews using AMSTAR 2.0 and Level of Evidence (LOE) using SIGN Methods:

\begin{tabular}{ccccccccccccccccccc}
\hline $\begin{array}{c}\text { Reference } \\
\text { number }\end{array}$ & $\mathbf{1}$ & $\mathbf{2}$ & $\mathbf{3}$ & $\mathbf{4}$ & $\mathbf{5}$ & $\mathbf{6}$ & $\mathbf{7}$ & $\mathbf{8}$ & $\mathbf{9}$ & $\mathbf{1 0}$ & $\mathbf{1 1}$ & $\mathbf{1 2}$ & $\mathbf{1 3}$ & $\mathbf{1 4}$ & $\mathbf{1 5}$ & $\mathbf{1 6}$ & \multirow{2}{*}{ LOE } \\
\hline $\mathrm{SR} 1$ & $\mathrm{Y}$ & $\mathrm{N}$ & $\mathrm{Y}$ & $\mathrm{N}$ & $\mathrm{Y}$ & $\mathrm{Y}$ & $\mathrm{Y}$ & $\mathrm{P}$ & $\mathrm{Y}$ & $\mathrm{N}$ & $\mathrm{Y}$ & $\mathrm{Y}$ & $\mathrm{Y}$ & $\mathrm{Y}$ & $\mathrm{Y}$ & $\mathrm{Y}$ & $1++$ \\
\hline
\end{tabular}

1. Did the research questions and inclusion criteria for the review include the components of PICO? 2. Did the report of the review contain an explicit statement that the review methods were established prior to the conduct of the review and did the report justify any significant deviations from the protocol? 3. Did the review authors explain their selection of the study designs for inclusion in the review? 4. Did the review authors use a comprehensive literature search strategy? 5. Did the review authors perform study selection in duplicate? 6. Did the review authors perform data extraction in duplicate? 7. Did the review authors provide a list of excluded studies and justify the exclusions? 8. Did the review authors describe the included studies in adequate detail? 9. Did the review authors use a satisfactory technique for assessing the risk of bias (RoB) in individual studies that were included in the review? 10. Did the review authors report on the sources of funding for the studies included in the review? 11. If meta-analysis was performed did the review authors use appropriate methods for statistical combination of results? 12. If meta-analysis was performed, did the review authors assess the potential impact of RoB in individual studies on the results of the meta-analysis or other evidence synthesis? 13. Did the review authors account for RoB in individual studies when interpreting/ discussing the results of the review? 14. Did the review authors provide a satisfactory explanation for, and discussion of, any heterogeneity observed in the results of the review? 15. If they performed quantitative synthesis did the review authors carry out an adequate investigation of publication bias (small study bias) and discuss its likely impact on the results of the review? 16. Did the review authors report any potential sources of conflict of interest, including any funding they received for conducting the review?

Y, yes; N, no; P, partial yes.

\section{ASSESSMENT OF CARDIAC REHABILITATION}

The following is the basic searching strategy used for cardiac rehabilitation assessment in the Cochrane Library, combined with the specific searching strategy for each question using AND.

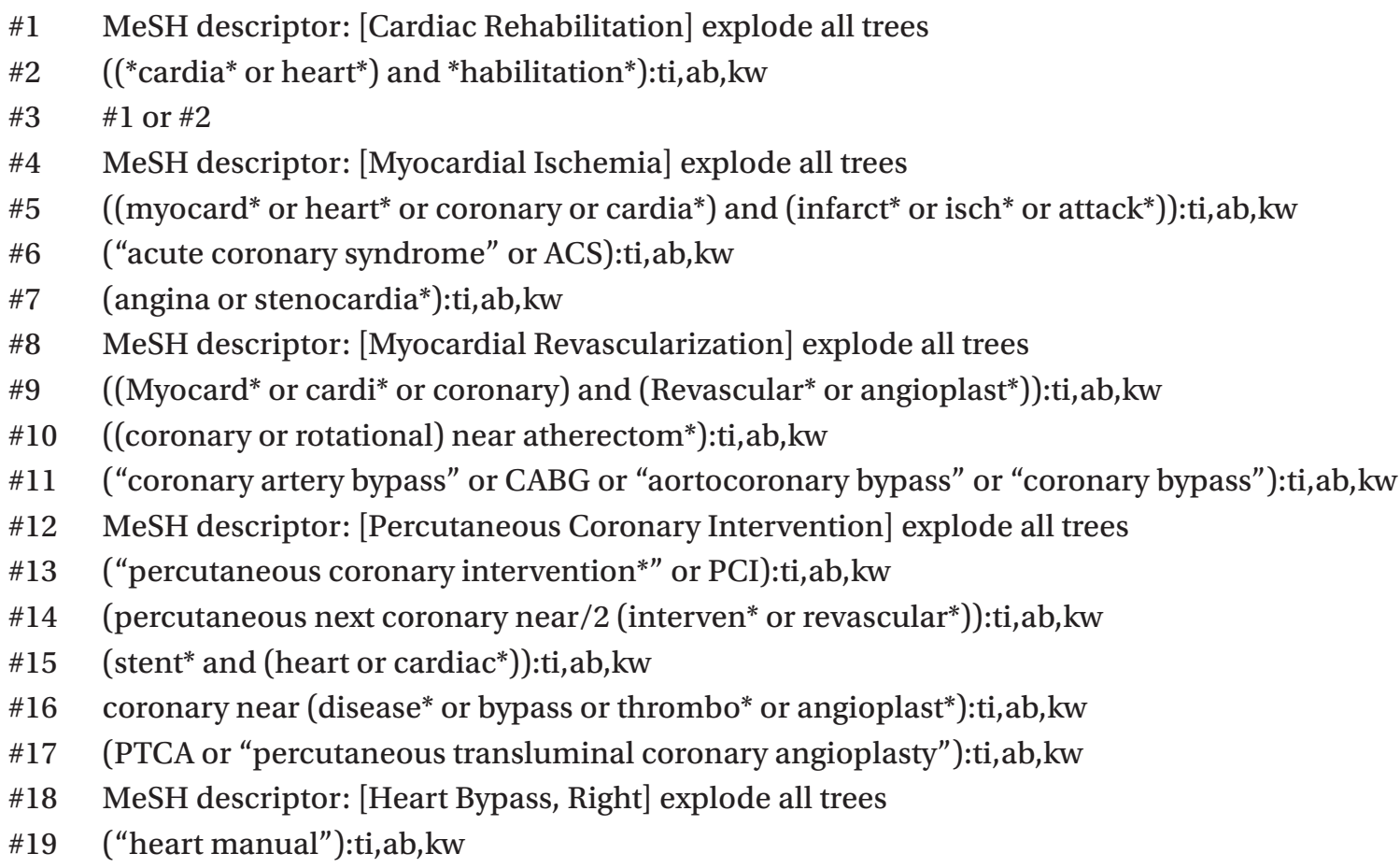




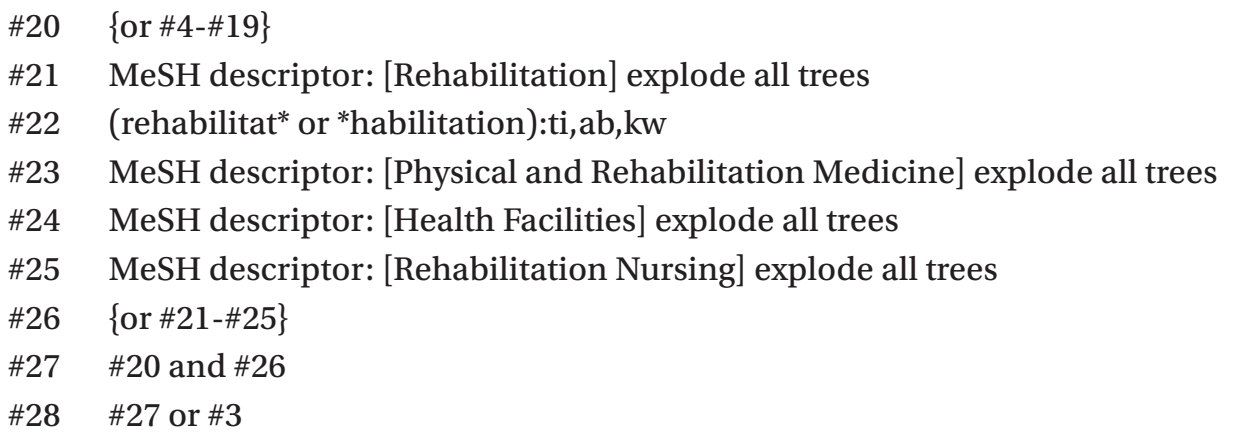

The following is the final Cochrane Library searching strategy used for KQ7 ("Is individualized cardiac rehabilitation program more effective than the existing fixed cardiac rehabilitation program?") including the basic CR assessment searching strategy:

\section{Searching Strategies}

\#29 ((individual* or personal* or "patient centred" or "patient centered" or "person centred" or "person centered") near/5 need*):ti,ab,kw

\#30 (tailored near/6 need*):ti,ab,kw

\#31 (standard* near/6 program*):ti,ab,kw

\#32 \{or \#29-\#31\}

\#33 \#28 and \#32 Publication Year from 2016 to 2018

Flow Chart of the Study Selection Process

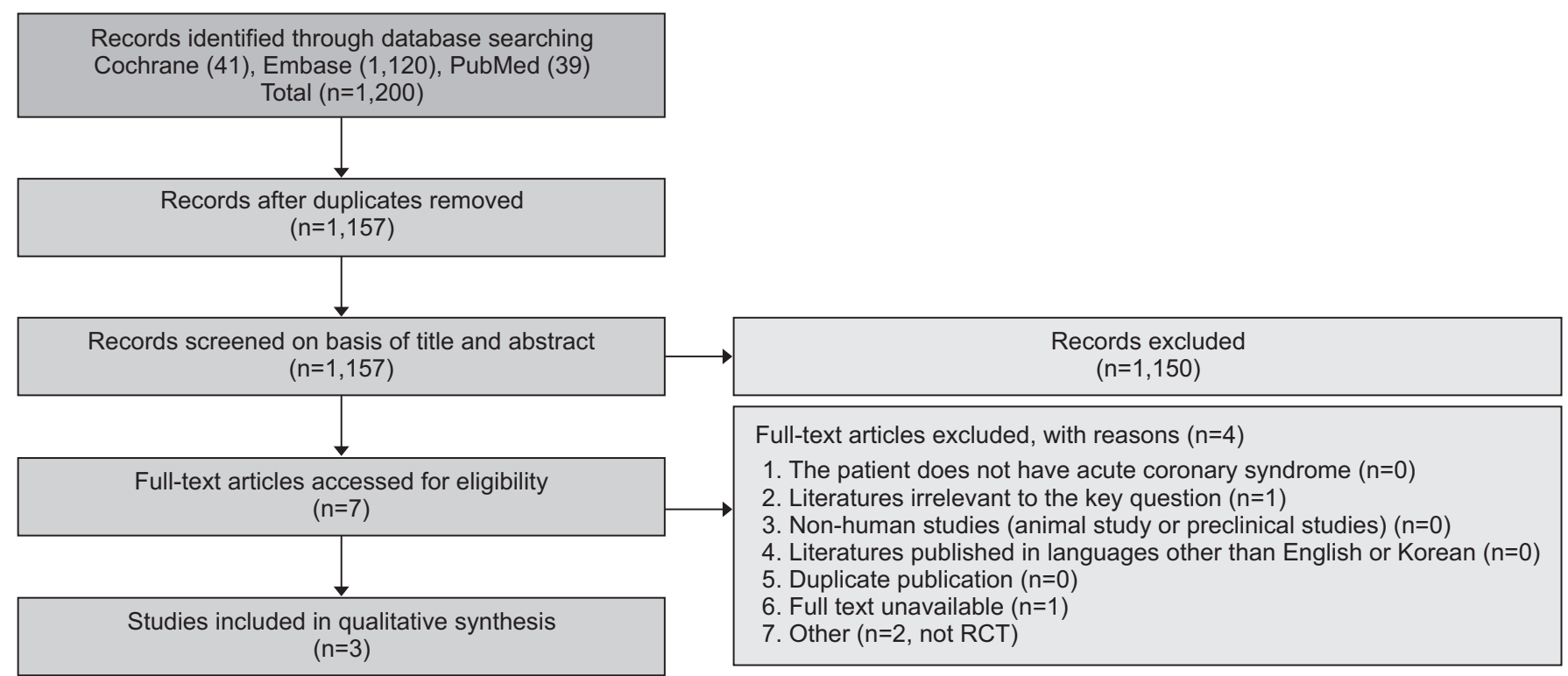


Finally Included Studies

\begin{tabular}{cc}
$\begin{array}{c}\text { Reference } \\
\text { number }\end{array}$ & Article \\
\hline RCT1 & $\begin{array}{c}\text { Weibel L, Massarotto P, Hediger H, Mahrer-Imhof R. Early education and counselling of patients with } \\
\text { acute coronary syndrome: a pilot study for a randomized controlled trial. Eur J Cardiovasc Nurs }\end{array}$ \\
& $\begin{array}{c}\text { 2016;15:213-22. } \\
\text { RCT2 }\end{array}$ \\
$\begin{array}{c}\text { Fors A, Swedberg K, Ulin K, Wolf A, Ekman I. Effects of person-centred care after an event of acute coro- } \\
\text { nary syndrome: two-year follow-up of a randomized controlled trial. Int J Cardiol 2017;249:42-7. }\end{array}$ \\
RCT3 & $\begin{array}{c}\text { Hill K, Walwyn R, Camidge MD. Murray J, Meads MD, Reynolds G, et al. A randomised feasibility trial of } \\
\text { a new lifestyle referral assessment versus usual assessment in an acute cardiology setting. J Cardiovasc } \\
\text { Nurs 2016;31:507-16. }\end{array}$ \\
\hline
\end{tabular}

RCT, randomized controlled trial.

Evidence Table for Assessment of Risk of Bias and Quality

Methodological Quality of Randomized Control Trials (RCT) using Cochrane Risk of Bias (RoB) tool and Level of Evidence (LOE) using SIGN Methods

\begin{tabular}{ccccccccc}
\hline $\begin{array}{c}\text { Reference } \\
\text { number }\end{array}$ & $\mathbf{1}$ & $\mathbf{2}$ & $\mathbf{3}$ & $\mathbf{4}$ & $\mathbf{5}$ & $\mathbf{6}$ & $\mathbf{7}$ & \multirow{2}{*}{ LOE } \\
\hline RCT1 & L & L & H & H & L & L & L & $1+$ \\
RCT2 & L & L & H & U & L & L & L & $1+$ \\
RCT3 & L & U & H & U & H & U & L & $1+$ \\
\hline
\end{tabular}

1. Random sequence generation: selection bias (biased allocation to interventions) due to inadequate generation of a randomized. 2. Allocation concealment: selection bias (biased allocation to interventions) due to inadequate concealment of allocations prior to assignment. 3. Blinding of participants and personnel: performance bias due to knowledge of the allocated interventions by participants and personnel during the study. 4. Blinding of outcome assessment: detection bias due to knowledge of the allocated interventions by outcome assessors. 5 . Incomplete outcome data: attrition bias due to amount, nature or handling of incomplete outcome data. 6. Selective reporting: reporting bias due to selective outcome reporting. 7. Other bias: bias due to problems not covered elsewhere in the table.

$\mathrm{L}$, low risk of bias; $\mathrm{H}$, high risk of bias; $\mathrm{U}$, unclear risk of bias.

KQ8 ("Should psychological interventions concerning anxiety, depression, and stress be included in the cardiac rehabilitation program?”) including the basic CR assessment searching strategy:

\section{Searching Strategies}

\#1 ((matched or stepped) near/3 care):ti,ab,kw

\#2 (tier or tiers or tiered):ti,ab,kw

\#3 (level* near/2 intervention*):ti,ab,kw

\#4 (step* up or step* down):ti,ab,kw

\#5 (matrix and psych*):ti,ab,kw

\#6 psychologist*:ti,ab,kw

\#7 MeSH descriptor: [Psychology] explode all trees

\#8 psycholog*:ti,ab,kw

\#9 (psychosocial or "psycho social"):ti,ab,kw

\#10 (stress near manage*):ti,ab,kw

\#11 (depress* or low next mood*):ti,ab,kw

\#12 \{or \#1-\#11\} 
Chul Kim, et al.

Flow Chart of Study Selection Process

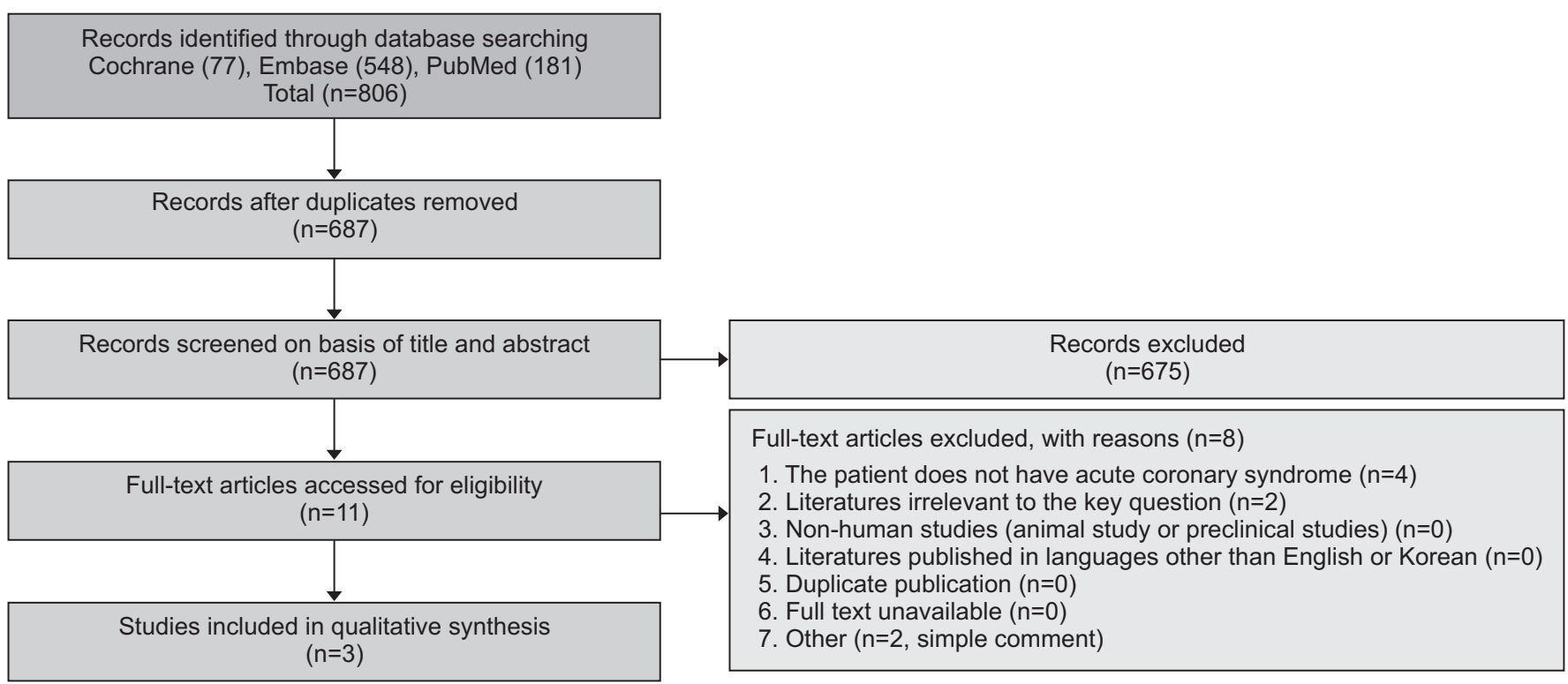

Finally Included Studies

\begin{tabular}{cc}
\hline $\begin{array}{c}\text { Reference } \\
\text { number }\end{array}$ & Article \\
\hline SR1 & $\begin{array}{c}\text { Klainin-Yobas P, Ng SH, Stephen PDM, Lau Y. Efficacy of psychosocial interventions on psychological } \\
\text { outcomes among people with cardiovascular diseases: a systematic review and meta-analysis. Patient } \\
\text { Educ Couns 2016;99:512-21. }\end{array}$ \\
SR2 & $\begin{array}{c}\text { Richards SH, Anderson L, Jenkinson CE, Whalley B, Rees K, Davies P, et al. Psychological interventions } \\
\text { for coronary heart disease. Cochrane Database Syst Rev 2017;4:CD002902. }\end{array}$ \\
RCT1 & $\begin{array}{c}\text { von Kanel R, Barth J, Princip M, Meister-Langraf RE, Schmid JP, Znoj H, et al. Early psychological coun- } \\
\text { seling for the prevention of posttraumatic stress induced by acute coronary syndrome: the MI-SPRINT } \\
\text { randomized controlled trial. Psychother Psychosom 2018;87:75-84. }\end{array}$ \\
\hline
\end{tabular}

SR, systematic reviews; RCT, randomized controlled trial. 
Evidence Table for Assessment of Risk of Bias and Quality

Methodological Quality of Systematic Reviews using AMSTAR 2.0 and Level of Evidence (LOE) using SIGN Methods

\begin{tabular}{ccccccccccccccccccc}
\hline $\begin{array}{c}\text { Reference } \\
\text { number }\end{array}$ & $\mathbf{1}$ & $\mathbf{2}$ & $\mathbf{3}$ & $\mathbf{4}$ & $\mathbf{5}$ & $\mathbf{6}$ & $\mathbf{7}$ & $\mathbf{8}$ & $\mathbf{9}$ & $\mathbf{1 0}$ & $\mathbf{1 1}$ & $\mathbf{1 2}$ & $\mathbf{1 3}$ & $\mathbf{1 4}$ & $\mathbf{1 5}$ & $\mathbf{1 6}$ & \multirow{2}{*}{ LOE } \\
\hline SR1 & $\mathrm{N}$ & $\mathrm{N}$ & $\mathrm{Y}$ & $\mathrm{N}$ & $\mathrm{Y}$ & $\mathrm{Y}$ & $\mathrm{N}$ & $\mathrm{Y}$ & $\mathrm{P}$ & $\mathrm{N}$ & $\mathrm{Y}$ & $\mathrm{N}$ & $\mathrm{N}$ & $\mathrm{N}$ & $\mathrm{Y}$ & $\mathrm{Y}$ & $1+$ \\
SR2 & $\mathrm{Y}$ & $\mathrm{Y}$ & $\mathrm{Y}$ & $\mathrm{Y}$ & $\mathrm{Y}$ & $\mathrm{Y}$ & $\mathrm{Y}$ & $\mathrm{Y}$ & $\mathrm{Y}$ & $\mathrm{Y}$ & $\mathrm{Y}$ & $\mathrm{Y}$ & $\mathrm{Y}$ & $\mathrm{Y}$ & $\mathrm{Y}$ & $\mathrm{Y}$ & $1++$ \\
\hline
\end{tabular}

1. Did the research questions and inclusion criteria for the review include the components of PICO? 2. Did the report of the review contain an explicit statement that the review methods were established prior to the conduct of the review and did the report justify any significant deviations from the protocol? 3. Did the review authors explain their selection of the study designs for inclusion in the review? 4. Did the review authors use a comprehensive literature search strategy? 5. Did the review authors perform study selection in duplicate? 6. Did the review authors perform data extraction in duplicate? 7. Did the review authors provide a list of excluded studies and justify the exclusions? 8. Did the review authors describe the included studies in adequate detail? 9. Did the review authors use a satisfactory technique for assessing the risk of bias (RoB) in individual studies that were included in the review? 10. Did the review authors report on the sources of funding for the studies included in the review? 11. If meta-analysis was performed did the review authors use appropriate methods for statistical combination of results? 12. If meta-analysis was performed, did the review authors assess the potential impact of RoB in individual studies on the results of the meta-analysis or other evidence synthesis? 13. Did the review authors account for RoB in individual studies when interpreting/ discussing the results of the review? 14. Did the review authors provide a satisfactory explanation for, and discussion of, any heterogeneity observed in the results of the review? 15. If they performed quantitative synthesis did the review authors carry out an adequate investigation of publication bias (small study bias) and discuss its likely impact on the results of the review? 16. Did the review authors report any potential sources of conflict of interest, including any funding they received for conducting the review?

$\mathrm{Y}=\mathrm{yes}, \mathrm{N}=\mathrm{no}, \mathrm{P}=$ partial yes.

Methodological Quality of Randomized Control Trials (RCT) using RoB and Level of Evidence (LOE) using SIGN Methods

\begin{tabular}{ccccccccc}
\hline $\begin{array}{c}\text { Reference } \\
\text { number }\end{array}$ & $\mathbf{1}$ & $\mathbf{2}$ & $\mathbf{3}$ & $\mathbf{4}$ & $\mathbf{5}$ & $\mathbf{6}$ & $\mathbf{7}$ & \multirow{2}{*}{ LOE } \\
\hline RCT1 & L & H & H & H & L & L & L & $1-$ \\
\hline
\end{tabular}

1. Random sequence generation: selection bias (biased allocation to interventions) due to inadequate generation of a randomized. 2. Allocation concealment: selection bias (biased allocation to interventions) due to inadequate concealment of allocations prior to assignment. 3. Blinding of participants and personnel: performance bias due to knowledge of the allocated interventions by participants and personnel During the study. 4. Blinding of outcome assessment: detection bias due to knowledge of the allocated interventions by outcome assessors. 5 . Incomplete outcome data: attrition bias due to amount, nature or handling of incomplete outcome data. 6 . Selective reporting: reporting bias due to selective outcome reporting. 7. Other bias: bias due to problems not covered elsewhere in the table.

$\mathrm{L}$, low risk of bias; $\mathrm{H}$, high risk of bias; $\mathrm{U}$, unclear risk of bias.

The following is the final Cochrane Library searching strategy used for KQ9 ("Is cardiopulmonary exercise test necessary for cardiac rehabilitation?") and KQ10 ("Is submaximal exercise test such as the 6-minute walk test useful for cardiac rehabilitation?") including the basic CR assessment searching strategy:

\section{Searching Strategies: KQ9 \& KQ10}

\#1 ("cardiopulmonary exercise test" or "exercise stress test" or "submaximal exercise test" or "submaximal exercise stress test" or "six minute walk test" or "six minute cycle test" or "walk test" or "cycle test"):ti,ab,kw

\#2 MeSH descriptor: [Exercise Test] explode all trees

\#3 submaximal test:ti,ab,kw

\#4 $\{$ or \#1-\#3\}

\#5 \#4 
Chul Kim, et al.

Flow Chart of Study Selection Process: KQ9 \& KQ10

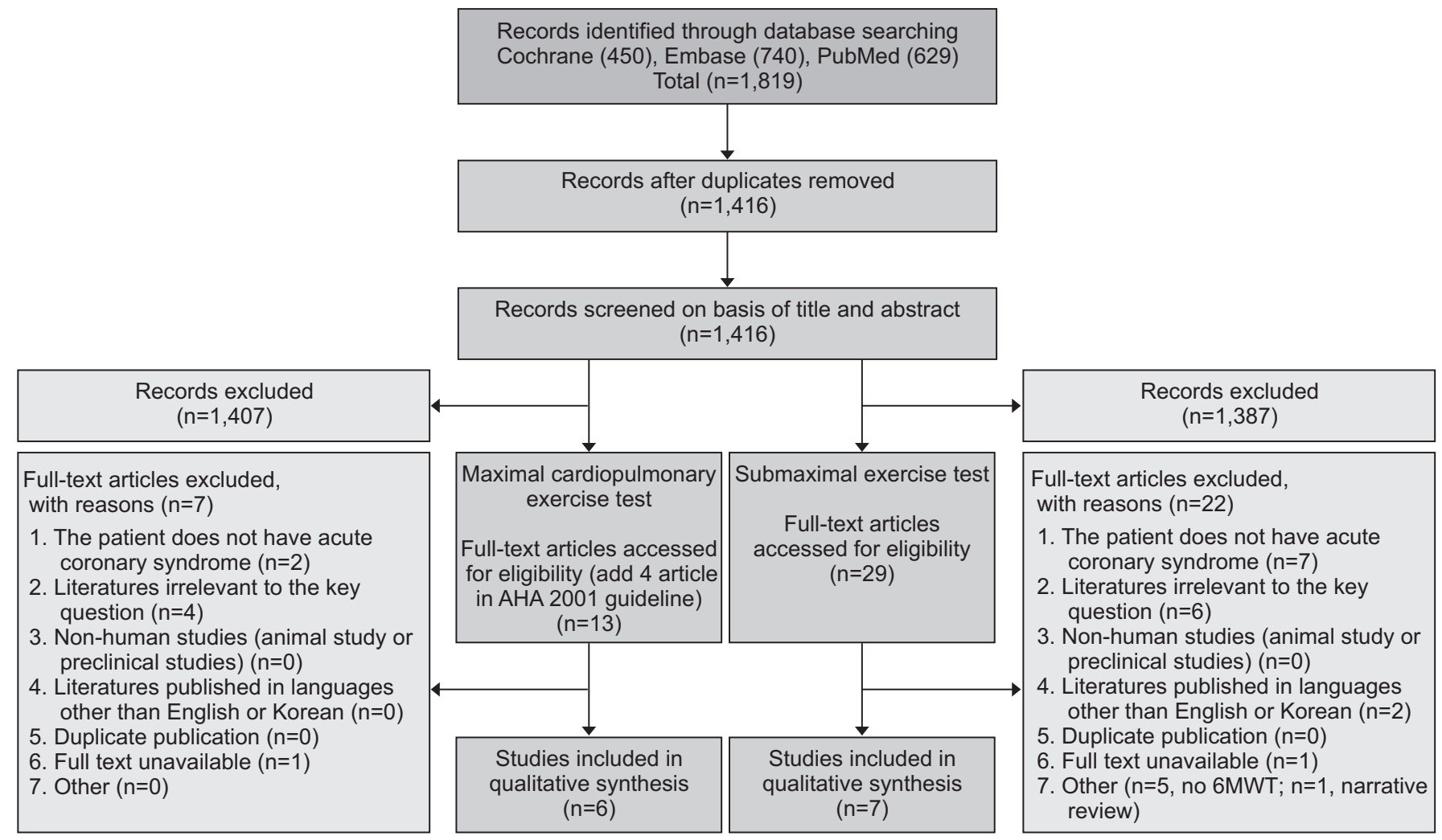

Finally Included Studies: KQ9

\begin{tabular}{cc|}
\hline $\begin{array}{c}\text { Reference } \\
\text { number }\end{array}$ & Article \\
\hline RCT1 & $\begin{array}{c}\text { Balady GJ, Leitschuh ML, Jacobs AK, Merrell D, Weiner DA, Ryan TJ. Safety and clinical use of exer- } \\
\text { cise testing one to three days after percutaneous transluminal coronary angioplasty. Am J Cardiol } \\
\text { 1992;69:1259-64. }\end{array}$ \\
\hline OS1 & $\begin{array}{c}\text { Safstrom K, Swahn E. Early symptom-limited exercise test for risk stratification in post menopausal wom- } \\
\text { en with unstable coronary artery disease. Eur Heart J 2000;21:230-8. }\end{array}$ \\
\hline OS2 & $\begin{array}{c}\text { Mark DB, Hlatky MA, Harrell FE Jr, Lee KL, Califf RM, Pryor DB. Exercise treadmill score for predicting } \\
\text { prognosis in coronary artery disease. Ann Intern Med 1987;106:793-800. }\end{array}$ \\
\hline OS3 & $\begin{array}{c}\text { Vanhees L, Fagard R, Thijs L, Staessen J, Amery A. Prognostic significance of peak exercise capacity in pa- } \\
\text { tients with coronary artery disease. J Am Coll Cardiol 1994;23:358-63. }\end{array}$ \\
\hline OS3 & $\begin{array}{c}\text { Kavanagh T, Mertens DJ, Hamm LF, Beyene J, Kennedy J, Corey P, et al. Prediction of long-term prognosis } \\
\text { in 12 169 men referred for cardiac rehabilitation. Circulation 2002;106:666-71. }\end{array}$ \\
\hline OS4 & $\begin{array}{c}\text { Kavanagh T, Mertens DJ, Hamm LF, Beyene J, Kennedy J, Corey P, et al. Peak oxygen intake and cardiac } \\
\text { mortality in women referred for cardiac rehabilitation. J Am Coll Cardiol 2003;42:2139-43. }\end{array}$ \\
\hline
\end{tabular}

RCT, randomized controlled trial; OS, observational study. 
Evidence Table for Assessment of Risk of Bias and Quality:KQ9

Methodological Quality of Randomized Control Trials (RCT) using RoB and Level of Evidence (LOE) using SIGN Methods

\begin{tabular}{ccccccccc}
\hline Reference & \multicolumn{9}{c}{ Quality items } & \multirow{2}{*}{ LOE } \\
\cline { 2 - 8 } number & $\mathbf{1}$ & $\mathbf{2}$ & $\mathbf{3}$ & $\mathbf{4}$ & $\mathbf{5}$ & $\mathbf{6}$ & $\mathbf{7}$ & \\
\hline RCT1 & H & H & H & H & L & L & L & $1-$ \\
\hline
\end{tabular}

1. Random sequence generation: selection bias (biased allocation to interventions) due to inadequate generation of a randomized. 2. Allocation concealment: selection bias (biased allocation to interventions) due to inadequate concealment of allocations prior to assignment. 3. Blinding of participants and personnel: performance bias due to knowledge of the allocated interventions by participants and personnel During the study. 4. Blinding of outcome assessment: detection bias due to knowledge of the allocated interventions by outcome assessors. 5 . Incomplete outcome data: attrition bias due to amount, nature or handling of incomplete outcome data. 6 . Selective reporting: reporting bias due to selective outcome reporting. 7. Other bias: bias due to problems not covered elsewhere in the table.

$\mathrm{L}$, low risk of bias; $\mathrm{H}$, high risk of bias; $\mathrm{U}$, unclear risk of bias.

Methodological Quality of Observation Study (OS) using The Risk of Bias Assessment Tool for Non-randomized Studies (RoBANS) and Level of Evidence (LOE) using SIGN Methods

\begin{tabular}{cccccccccc}
\hline $\begin{array}{c}\text { Reference } \\
\text { number }\end{array}$ & $\mathbf{1}$ & $\mathbf{2}$ & $\mathbf{3}$ & $\mathbf{4}$ & $\mathbf{5}$ & $\mathbf{6}$ & $\mathbf{7}$ & $\mathbf{8}$ & \multirow{2}{*}{ LOE } \\
\hline OS1 & L & L & L & L & L & L & U & L & $2++$ \\
OS2 & U & L & H & L & L & L & L & L & $2++$ \\
OS3 & U & L & L & L & L & L & L & L & $2++$ \\
OS4 & U & L & L & L & L & L & L & L & $2++$ \\
OS5 & U & L & L & L & L & L & L & L & $2++$ \\
\hline
\end{tabular}

1. Comparability = selection bias caused by inappropriate participant selection. 2 . Selection of participants = selection bias caused by inadequate selection of participants for intervention, exposure, or patient group. 3. Confounding variables $=$ Selection bias caused by inadequate confirmation and consideration of confounding variables. 4 . Exposure measurement $=$ performance bias caused by inadequate measurement of intervention or exposure. 5. Blinding of outcome assessment = detection bias caused by inadequate blinding of outcome assessment. 6 . Outcome assessment = detection bias caused by inappropriate outcome assessment method. 7 . Incomplete outcome data $=$ attrition bias caused by inappropriate handling of inappropriate data. 8 . Selective outcome reporting $=$ reporting bias caused by selective outcome reporting.

$\mathrm{L}$, low risk of bias; $\mathrm{H}$, high risk of bias; $\mathrm{U}$, unclear risk of bias. 


\begin{tabular}{ccc}
$\begin{array}{c}\text { Reference } \\
\text { number }\end{array}$ & Article \\
\hline SR1 & $\begin{array}{c}\text { Bellet RN, Adams L, Morris NR. The 6-minute walk test in outpatient cardiac rehabilitation: validity, reli- } \\
\text { ability and responsiveness. A systematic review. Physiotherapy 2012;98:277-86. }\end{array}$ \\
OS1 & $\begin{array}{c}\text { Wright DJ, Khan KM, Gossage EM, Saltissi S. Assessment of a low-intensity cardiac rehabilitation pro- } \\
\text { gramme using the six-minute walk test. Clin Rehabil 2001;15:119-24. }\end{array}$ \\
OS2 & $\begin{array}{c}\text { Nogueira PA, Leal AC, Pulz C, Nogueira ID, Filho JA. Clinical reliability of the } 6 \text { minute corridor walk test } \\
\text { performed within a week of a myocardial infarction. Int Heart J 2006;47:533-40. }\end{array}$ \\
OS3 & $\begin{array}{c}\text { Gremeaux M, Hannequin A, Laurent Y, Laroche D, Casillas JM, Gremeaux V. Usefulness of the 6-minute } \\
\text { walk test and the 200-metre fast walk test to individualize high intensity interval and continuous exercise } \\
\text { training in coronary artery disease patients after acute coronary syndrome: a pilot } \\
\text { controlled clinical study. Clin Rehabil 2011;25:844-55. }\end{array}$ \\
\hline OS4 & $\begin{array}{c}\text { Hanson LC, McBurney H, Taylor NF. The retest reliability of the six-minute walk test in patients referred to } \\
\text { a cardiac rehabilitation programme. Physiother Res Int 2012;17:55-61. }\end{array}$ \\
OS5 & $\begin{array}{c}\text { Diniz LS, Neves VR, Starke AC, Barbosa MP, Britto RR, Ribeiro AL. Safety of early performance of the } \\
\text { six-minute walk test following acute myocardial infarction: a cross-sectional study. Braz J Phys Ther } \\
\text { 2017;21:167-74. }\end{array}$ \\
\hline OS6 & $\begin{array}{c}\text { Harris KM, Anderson DR, Landers JD, Emery CF. Utility of walk tests in evaluating functional status among } \\
\text { participants in an outpatient cardiac rehabilitation program. J Cardiopulm Rehabil Prev 2017;37:329-33. }\end{array}$ \\
\hline
\end{tabular}

SR, systematic reviews; OS, observational study.

Evidence Table for Assessment of Risk of Bias and Quality: KQ10

Evidence Table for Assessment of Risk of Bias and Quality

Methodological Quality of Systematic Reviews using AMSTAR 2.0 and Level of Evidence (LOE) using SIGN Methods

\begin{tabular}{ccccccccccccccccccc}
\hline $\begin{array}{c}\text { Reference } \\
\text { number }\end{array}$ & $\mathbf{1}$ & $\mathbf{2}$ & $\mathbf{3}$ & $\mathbf{4}$ & $\mathbf{5}$ & $\mathbf{6}$ & $\mathbf{7}$ & $\mathbf{8}$ & $\mathbf{9}$ & $\mathbf{1 0}$ & $\mathbf{1 1}$ & $\mathbf{1 2}$ & $\mathbf{1 3}$ & $\mathbf{1 4}$ & $\mathbf{1 5}$ & $\mathbf{1 6}$ & LOE \\
\hline SR1 & $\mathrm{Y}$ & $\mathrm{Y}$ & $\mathrm{Y}$ & $\mathrm{Y}$ & $\mathrm{Y}$ & $\mathrm{Y}$ & $\mathrm{P}$ & $\mathrm{Y}$ & $\mathrm{Y}$ & $\mathrm{N}$ & $\mathrm{Y}$ & $\mathrm{Y}$ & $\mathrm{N}$ & $\mathrm{Y}$ & $\mathrm{N}$ & $\mathrm{Y}$ & $2+$ \\
\hline
\end{tabular}

1. Did the research questions and inclusion criteria for the review include the components of PICO? 2. Did the report of the review contain an explicit statement that the review methods were established prior to the conduct of the review and did the report justify any significant deviations from the protocol? 3. Did the review authors explain their selection of the study designs for inclusion in the review? 4. Did the review authors use a comprehensive literature search strategy? 5. Did the review authors perform study selection in duplicate? 6. Did the review authors perform data extraction in duplicate? 7. Did the review authors provide a list of excluded studies and justify the exclusions? 8. Did the review authors describe the included studies in adequate detail? 9. Did the review authors use a satisfactory technique for assessing the risk of bias (RoB) in individual studies that were included in the review? 10. Did the review authors report on the sources of funding for the studies included in the review? 11. If meta-analysis was performed did the review authors use appropriate methods for statistical combination of results? 12. If meta-analysis was performed, did the review authors assess the potential impact of RoB in individual studies on the results of the meta-analysis or other evidence synthesis? 13. Did the review authors account for RoB in individual studies when interpreting/ discussing the results of the review? 14. Did the review authors provide a satisfactory explanation for, and discussion of, any heterogeneity observed in the results of the review? 15. If they performed quantitative synthesis did the review authors carry out an adequate investigation of publication bias (small study bias) and discuss its likely impact on the results of the review? 16. Did the review authors report any potential sources of conflict of interest, including any funding they received for conducting the review?

Y, yes; N, no; P, partial yes. 
Methodological Quality of Observation Study (OS) using The Risk of Bias Assessment Tool for Non-randomized Studies (RoBANS) and Level of Evidence (LOE) using SIGN Methods

\begin{tabular}{|c|c|c|c|c|c|c|c|c|c|}
\hline \multirow{2}{*}{$\begin{array}{c}\text { Reference } \\
\text { number }\end{array}$} & \multicolumn{8}{|c|}{ Quality items } & \multirow{2}{*}{ LOE } \\
\hline & 1 & 2 & 3 & 4 & 5 & 6 & 7 & 8 & \\
\hline OS1 & $\mathrm{H}$ & $\mathrm{L}$ & $\mathrm{L}$ & $\mathrm{L}$ & $\mathrm{H}$ & $\mathrm{L}$ & $\mathrm{L}$ & $\mathrm{L}$ & $2+$ \\
\hline OS2 & $\mathrm{U}$ & $\mathrm{H}$ & $\mathrm{L}$ & U & $\mathrm{H}$ & $\mathrm{L}$ & $\mathrm{L}$ & $\mathrm{L}$ & $2+$ \\
\hline OS3 & $\mathrm{H}$ & $\mathrm{H}$ & $\mathrm{H}$ & $\mathrm{L}$ & $\mathrm{H}$ & $\mathrm{L}$ & $\mathrm{L}$ & $\mathrm{L}$ & $2-$ \\
\hline OS4 & $\mathrm{U}$ & $\mathrm{L}$ & $\mathrm{L}$ & $\mathrm{L}$ & $\mathrm{H}$ & $\mathrm{L}$ & $\mathrm{L}$ & $\mathrm{L}$ & $2+$ \\
\hline OS5 & $\mathrm{H}$ & $\mathrm{L}$ & $\mathrm{H}$ & $\mathrm{L}$ & $\mathrm{H}$ & $\mathrm{L}$ & $\mathrm{L}$ & $\mathrm{L}$ & $2+$ \\
\hline OS6 & $\mathrm{U}$ & $\mathrm{H}$ & $\mathrm{L}$ & $\mathrm{L}$ & $\mathrm{H}$ & $\mathrm{L}$ & $\mathrm{L}$ & $\mathrm{L}$ & $2++$ \\
\hline
\end{tabular}

1. Comparability = selection bias caused by inappropriate participant selection. 2 . Selection of participants = selection bias caused by inadequate selection of participants for intervention, exposure, or patient group. 3. Confounding variables $=$ Selection bias caused by inadequate confirmation and consideration of confounding variables. 4 . Exposure measurement $=$ performance bias caused by inadequate measurement of intervention or exposure. 5. Blinding of outcome assessment = detection bias caused by inadequate blinding of outcome assessment. 6 . Outcome assessment = detection bias caused by inappropriate outcome assessment method. 7 . Incomplete outcome data $=$ attrition bias caused by inappropriate handling of inappropriate data. 8 . Selective outcome reporting $=$ reporting bias caused by selective outcome reporting

$\mathrm{L}$, low risk of bias; $\mathrm{H}$, high risk of bias; $\mathrm{U}$, unclear risk of bias.

The following is the final Cochrane Library searching strategy used for KQ11 ("What measures effectively promote participation in cardiac rehabilitation?") and KQ12 ("What measures effectively increase physical activity compliance?") including the basic CR assessment searching strategy:

\section{Searching Strategies}

\#1 (increase* near/10 participat*):ti,ab,kw

\#2 (comply or complian*):ti,ab,kw

\#3 (remain* or adhere* or uptake or "take up" or "sign up" or "sign on" or "follow up" or engage* or attend* or maintenance*):ti,ab,kw

\#4 (enrollment or enrolment or enroling or enrolling):ti,ab,kw

\#5 (participat* or motivation* or uptake or referral or adherence or attend* or non-attend* or barrier* or engaging or engagement):ti,ab,kw

\#6 MeSH descriptor: [Health Services Accessibility] explode all trees

\#7 MeSH descriptor: [Patient Compliance] explode all trees

\#8 MeSH descriptor: [Referral and Consultation] explode all trees

\#9 MeSH descriptor: [Patient Satisfaction] explode all trees

\#10 MeSH descriptor: [Patient Participation] explode all trees

\#11 MeSH descriptor: [Self Efficacy] explode all trees

\#12 MeSH descriptor: [Motivation] explode all trees

$\# 13 \quad$ or \#1-\#12\} 
Chul Kim, et al.

Flow Chart of Study Selection Process: KQ11 \& KQ12

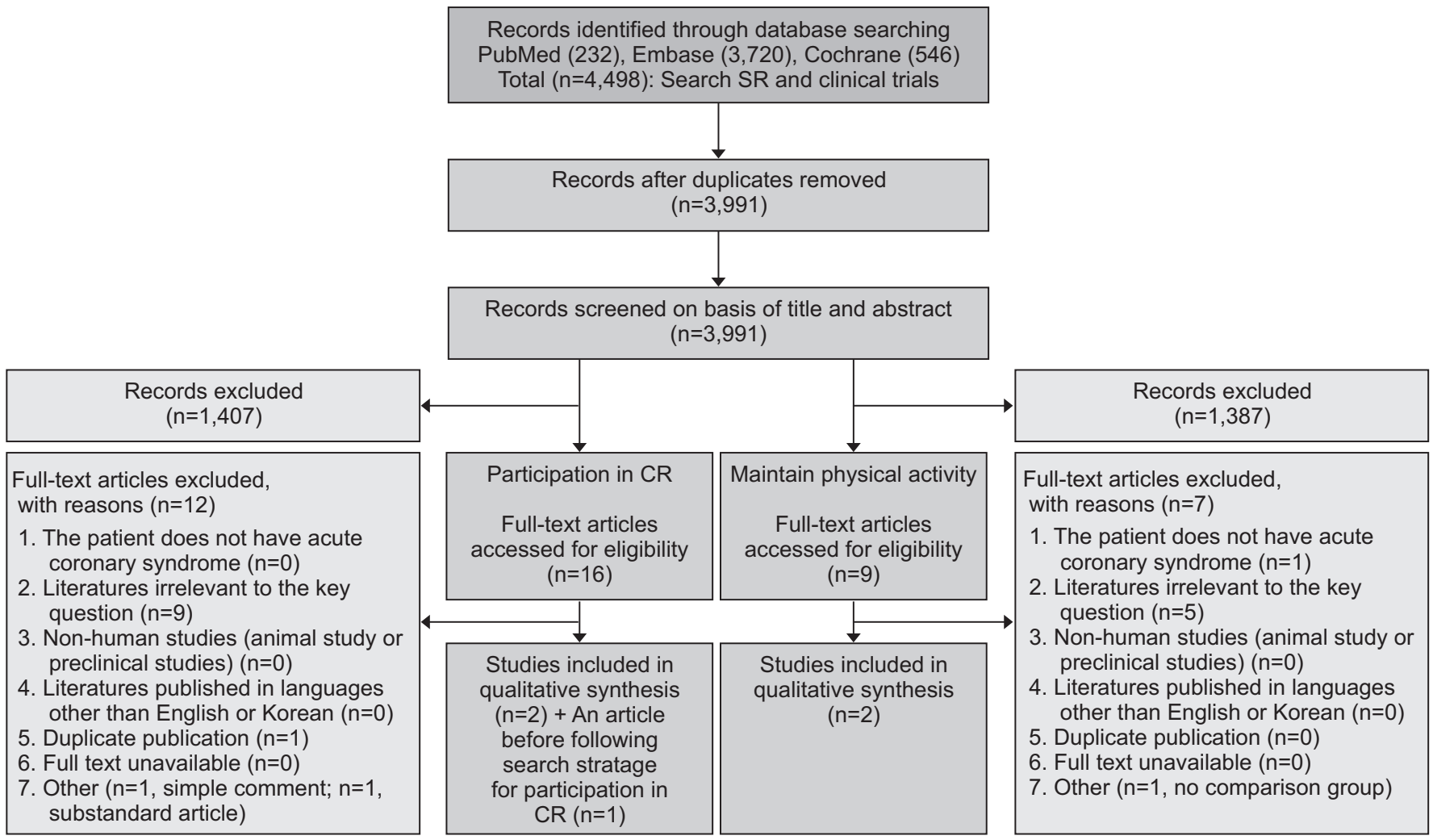

Finally Included Studies: KQ11

\begin{tabular}{ll}
$\begin{array}{c}\text { Reference } \\
\text { number }\end{array}$ & Article \\
\hline
\end{tabular}

SR1 Grace SL, Chessex C, Arthur H, Chan S, Cyr C, Dafoe W, et al. Systematizing inpatient referral to cardiac rehabilitation 2010: Canadian Association of Cardiac Rehabilitation and Canadian Cardiovascular Society joint position paper endorsed by the Cardiac Care Network of Ontario. Can J Cardiol 2011;27:192-9.

RCT1 Grace SL, Midence L, Oh P, Brister S, Chessex C, Stewart DE, et al. Cardiac rehabilitation program adherence and functional capacity among women: a randomized controlled trial. Mayo Clin Proc 2016;91:140-8.

RCT2 Lynggaard V, Nielsen CV, Zwisler AD, Taylor RS, May O. The patient education: learning and coping strategies. Improves adherence in cardiac rehabilitation (LC-REHAB): a randomised controlled trial. Int J Cardiol 2017;236:65-70.

SR, systematic reviews; RCT, randomized controlled trial. 
Evidence Table for Assessment of Risk of Bias and Quality: KQ11

Systematic Reviews (SR)

\begin{tabular}{|c|c|c|c|c|c|c|c|c|c|c|c|c|c|c|c|c|c|}
\hline \multirow{2}{*}{$\begin{array}{c}\text { Reference } \\
\text { number }\end{array}$} & \multicolumn{16}{|c|}{ Quality items } & \multirow{2}{*}{ LOE } \\
\hline & 1 & 2 & 3 & 4 & 5 & 6 & 7 & 8 & 9 & 10 & 11 & 12 & 13 & 14 & 15 & 16 & \\
\hline SR1 & $\mathrm{Y}$ & $\mathrm{Y}$ & $\mathrm{Y}$ & $\mathrm{Y}$ & $\mathrm{Y}$ & $\mathrm{Y}$ & $\mathrm{N}$ & $\mathrm{N}$ & $\mathrm{N}$ & $\mathrm{N}$ & $\mathrm{N}$ & $\mathrm{N}$ & $\mathrm{N}$ & $\mathrm{P}$ & $\mathrm{N}$ & $\mathrm{N}$ & $2-$ \\
\hline
\end{tabular}

1. Did the research questions and inclusion criteria for the review include the components of PICO? 2. Did the report of the review contain an explicit statement that the review methods were established prior to the conduct of the review and did the report justify any significant deviations from the protocol? 3. Did the review authors explain their selection of the study designs for inclusion in the review? 4. Did the review authors use a comprehensive literature search strategy? 5. Did the review authors perform study selection in duplicate? 6. Did the review authors perform data extraction in duplicate? 7. Did the review authors provide a list of excluded studies and justify the exclusions? 8 . Did the review authors describe the included studies in adequate detail? 9. Did the review authors use a satisfactory technique for assessing the risk of bias (RoB) in individual studies that were included in the review? 10. Did the review authors report on the sources of funding for the studies included in the review? 11. If meta-analysis was performed did the review authors use appropriate methods for statistical combination of results? 12. If meta-analysis was performed, did the review authors assess the potential impact of RoB in individual studies on the results of the meta-analysis or other evidence synthesis? 13. Did the review authors account for RoB in individual studies when interpreting/ discussing the results of the review? 14. Did the review authors provide a satisfactory explanation for, and discussion of, any heterogeneity observed in the results of the review? 15. If they performed quantitative synthesis did the review authors carry out an adequate investigation of publication bias (small study bias) and discuss its likely impact on the results of the review? 16. Did the review authors report any potential sources of conflict of interest, including any funding they received for conducting the review?

Y, yes; N, no; P, partial yes.

Randomized Control Trials (RCTs)

\begin{tabular}{ccccccccc}
\hline $\begin{array}{c}\text { Reference } \\
\text { number }\end{array}$ & $\mathbf{1}$ & $\mathbf{2}$ & $\mathbf{3}$ & $\mathbf{4}$ & $\mathbf{5}$ & $\mathbf{6}$ & $\mathbf{7}$ & \multirow{2}{*}{ LOE } \\
\hline RCT1 & $\mathrm{L}$ & $\mathrm{L}$ & $\mathrm{L}$ & $\mathrm{L}$ & $\mathrm{H}$ & $\mathrm{L}$ & $\mathrm{L}$ & $1++$ \\
RCT2 & $\mathrm{L}$ & $\mathrm{U}$ & $\mathrm{U}$ & $\mathrm{U}$ & $\mathrm{L}$ & $\mathrm{L}$ & $\mathrm{L}$ & $1+$ \\
\hline
\end{tabular}

1. Random sequence generation: selection bias (biased allocation to interventions) due to inadequate generation of a randomized. 2. Allocation concealment: selection bias (biased allocation to interventions) due to inadequate concealment of allocations prior to assignment. 3. Blinding of participants and personnel: performance bias due to knowledge of the allocated interventions by participants and personnel during the study. 4. Blinding of outcome assessment: detection bias due to knowledge of the allocated interventions by outcome assessors. 5. Incomplete outcome data: attrition bias due to amount, nature or handling of incomplete outcome data. 6. Selective reporting: reporting bias due to selective outcome reporting. 7. Other bias: bias due to problems not covered elsewhere in the table.

$\mathrm{L}$, low risk of bias; $\mathrm{H}$, high risk of bias; $\mathrm{U}$, unclear risk of bias.

Finally Included Studies: KQ12

\begin{tabular}{cl}
$\begin{array}{c}\text { Reference } \\
\text { number }\end{array}$ & \multicolumn{1}{c}{ Article } \\
\hline RCT1 & $\begin{array}{c}\text { Fournier M, Radel R, Bailly L, Pradier C, Fabre R, Fuch A, et al. "As du Coeur" study: a randomized } \\
\text { controlled trial on physical activity maintenance in cardiovascular patients. BMC Cardiovasc Disord }\end{array}$ \\
& $\begin{array}{c}2018 ; 18: 77 . \\
\text { RCT2 }\end{array}$ \\
$\begin{array}{l}\text { Ter Hoeve N, Sunamura M, Stam HJ, Boersma E, Geleijnse ML, van Domburg RT, et al. Effects of two } \\
\text { behavioral cardiac rehabilitation interventions on physical activity: a randomized controlled trial. } \\
\text { Int J Cardiol 2018;255:221-8. }\end{array}$ \\
\hline
\end{tabular}

RCT, randomized controlled trial. 
Evidence Table for Assessment of Risk of Bias and Quality: KQ12

Randomized Control Trials (RCTs)

\begin{tabular}{ccccccccc}
\hline Reference & \multicolumn{9}{c}{ Quality items } & \multirow{2}{*}{ LOE } \\
\cline { 2 - 8 } number & $\mathbf{1}$ & $\mathbf{2}$ & $\mathbf{3}$ & $\mathbf{4}$ & $\mathbf{5}$ & $\mathbf{6}$ & $\mathbf{7}$ & \\
\hline RCT1 & L & L & L & L & L & L & H & $1+$ \\
RCT2 & L & L & H & H & L & L & L & $1+$ \\
\hline
\end{tabular}

1. Random sequence generation: selection bias (biased allocation to interventions) due to inadequate generation of a randomized. 2. Allocation concealment: selection bias (biased allocation to interventions) due to inadequate concealment of allocations prior to assignment. 3. Blinding of participants and personnel: performance bias due to knowledge of the allocated interventions by participants and personnel during the study. 4. Blinding of outcome assessment: detection bias due to knowledge of the allocated interventions by outcome assessors. 5. Incomplete outcome data: attrition bias due to amount, nature or handling of incomplete outcome data. 6. Selective reporting: reporting bias due to selective outcome reporting. 7. Other bias: bias due to problems not covered elsewhere in the table.

$\mathrm{L}$, low risk of bias; $\mathrm{H}$, high risk of bias; $\mathrm{U}$, unclear risk of bias.

\section{EXERCISE THERAPY FOR CARDIAC REHABILITATION}

The following is the common searching strategy for KQ13 ("When should patients begin cardiac rehabilitation after CABG?") combined with the specific searching strategies for each question shown below:

\#1 MeSH descriptor: [Myocardial Ischemia] explode all trees

\#2 ((myocard* or heart* or coronary or cardia*) and (infarct* or isch* or attack*)):ti,ab,kw

\#3 ("acute coronary syndrome" or ACS):ti,ab,kw

\#4 (angina or stenocardia*):ti,ab,kw

\#5 $\quad$ or \#1-\#4 $\}$

\#6 MeSH descriptor: [Cardiac Surgical Procedures] explode all trees

\#7 ("coronary artery bypass" or "CABG" or "aortocoronary bypass" or "coronary bypass"):ti,ab,kw

\#8 ("coronary" near "arter*" near "bypass"):ti,ab,kw

$\# 9 \quad\{$ or \#6-\#8\}

$\# 10 \quad \# 5$ and $\# 9$

The following is the specific searching strategy for KQ13-1, "CABG ambulation":

\#11 MeSH descriptor: [Ambulatory Care] explode all trees

\#12 MeSH descriptor: [Early Ambulation] explode all trees

\#13 (ambulat* or mobili* or gait):ti,ab,kw

\#14 \{or \#11-\#13\}

\#15 \#10 and \#14

The following is the specific searching strategy for KQ13-2, "CABG aerobic and strengthening":

\#11 MeSH descriptor: [Exercise] explode all trees

\#12 MeSH descriptor: [Exercise Therapy] explode all trees

\#13 (("exercise*" or "train*" or fitness) and ("strength*" or "aerobic")):ti,ab,kw

\#14 (physical near (train* or activ*)):ti,ab,kw

\#15 ((physio or physic* or kinesio*) near therap*):ti,ab,kw 
\#16 ((interval or aerobic) near (train* or exercise* or run* or fitness)):ti,ab,kw

\#17 ((muscle* or resistan*) near (train* or activ* or strength* or exercise*)):ti,ab,kw

\#18 \{or \#11-\#17\}

$\# 19 \quad \# 10$ and \#18

The following is the specific searching strategy for KQ13-3, "CABG + Stretching":

\#11 MeSH descriptor: [Range of Motion, Articular] explode all trees

\#12 (stretch* or flexibilit*):ti,ab,kw

\#13 \#11 or \#12

\#14 \#10 and \#13

The following is the specific searching strategy for KQ13-4, "CABG + Respiration training":

\#11 (respirat* or inspirat*) near (train* or educat* or exercise* or physiotherap*):ti,ab,kw

\#12 spiromet*:ti,ab,kw

\#13 \#11 or \#12

$\# 14 \quad \# 10$ and \#13

The following is the specific searching strategy for KQ13-5. "CABG + Dysphagia":

\#11 MeSH descriptor: [Deglutition Disorders] explode all trees

\#12 (dysphagia or (swallow* near (difficult* or disorder))):ti,ab,kw

\#13 \#11 or \#12

\#14 \#10 and \#13

Flow Chart of Study Selection Process

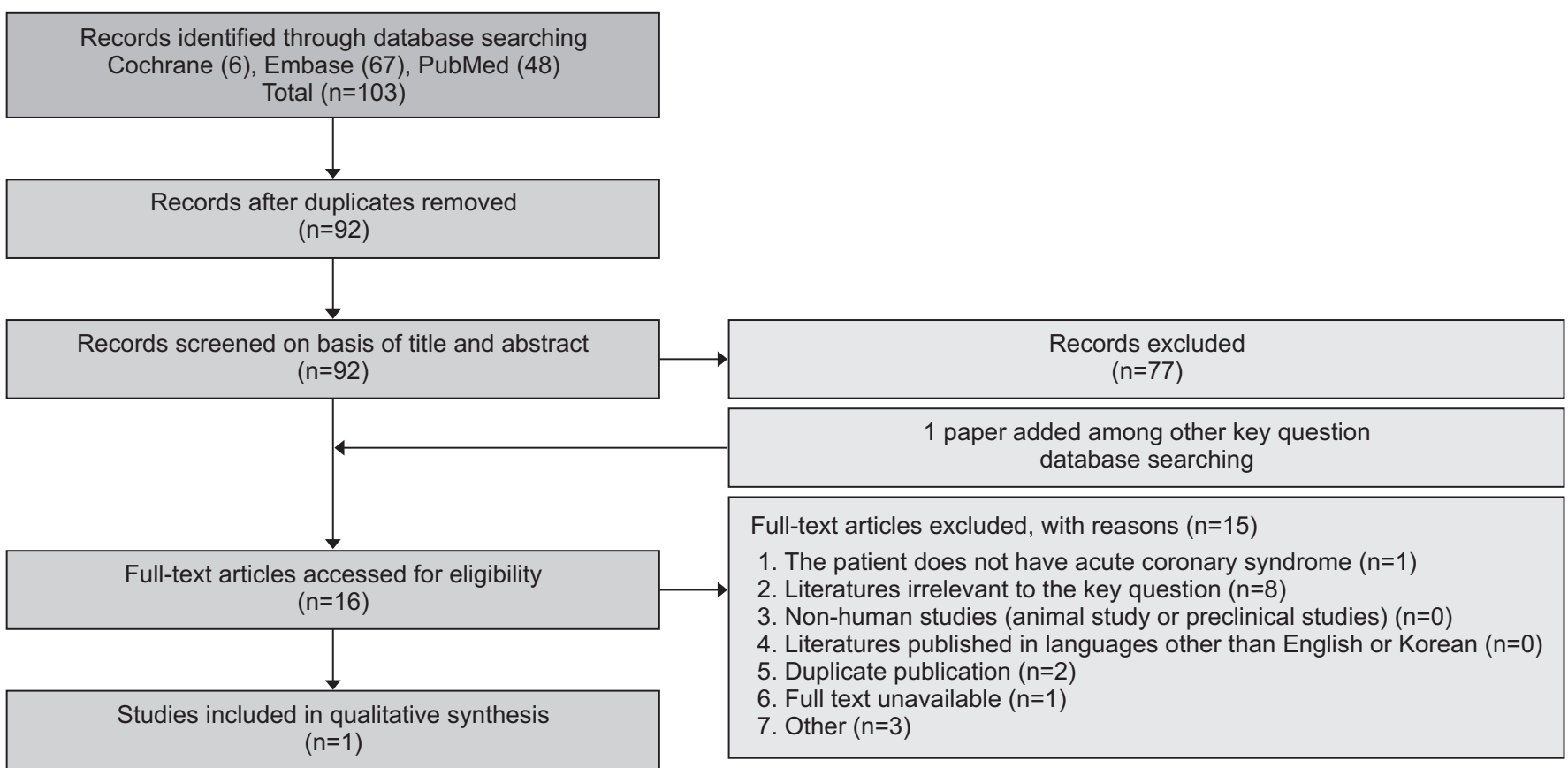


Finally Included Studies

\begin{tabular}{cc}
$\begin{array}{c}\text { Reference } \\
\text { number }\end{array}$ & Article \\
\hline SR1 & $\begin{array}{c}\text { Aldahash R, Al Dera HS. Physical therapy program improves the physiological impact towards better } \\
\text { quality of life and low cardiac risk factors in patients following coronary artery bypass grafting: system- } \\
\text { atic review. Acta Medica Int 2016;3:185-95. }\end{array}$ \\
\hline
\end{tabular}

SR, systematic reviews.

Evidence Table for Assessment of Risk of Bias and Quality

Methodological Quality of Systematic Reviews using AMSTAR 2.0 and Level of Evidence (LOE) using SIGN Methods

\begin{tabular}{cccccccccccccccccc}
\hline $\begin{array}{c}\text { Reference } \\
\text { number }\end{array}$ & $\mathbf{1}$ & $\mathbf{2}$ & $\mathbf{3}$ & $\mathbf{4}$ & $\mathbf{5}$ & $\mathbf{6}$ & $\mathbf{7}$ & $\mathbf{8}$ & $\mathbf{9}$ & $\mathbf{1 0}$ & $\mathbf{1 1}$ & $\mathbf{1 2}$ & $\mathbf{1 3}$ & $\mathbf{1 4}$ & $\mathbf{1 5}$ & $\mathbf{1 6}$ & LOE \\
\hline SR1 & $\mathrm{Y}$ & $\mathrm{N}$ & $\mathrm{Y}$ & $\mathrm{P}$ & $\mathrm{N}$ & $\mathrm{N}$ & $\mathrm{N}$ & $\mathrm{P}$ & $\mathrm{N}$ & $\mathrm{N}$ & $\mathrm{N}$ & $\mathrm{M}$ & $\mathrm{M}$ & $\mathrm{N}$ & $\mathrm{M}$ & $\mathrm{Y}$ & $1-$ \\
\hline
\end{tabular}

1. Did the research questions and inclusion criteria for the review include the components of PICO? 2. Did the report of the review contain an explicit statement that the review methods were established prior to the conduct of the review and did the report justify any significant deviations from the protocol? 3. Did the review authors explain their selection of the study designs for inclusion in the review? 4. Did the review authors use a comprehensive literature search strategy? 5. Did the review authors perform study selection in duplicate? 6. Did the review authors perform data extraction in duplicate? 7. Did the review authors provide a list of excluded studies and justify the exclusions? 8 . Did the review authors describe the included studies in adequate detail? 9. Did the review authors use a satisfactory technique for assessing the risk of bias (RoB) in individual studies that were included in the review? 10. Did the review authors report on the sources of funding for the studies included in the review? 11. If meta-analysis was performed did the review authors use appropriate methods for statistical combination of results? 12. If meta-analysis was performed, did the review authors assess the potential impact of RoB in individual studies on the results of the meta-analysis or other evidence synthesis? 13. Did the review authors account for RoB in individual studies when interpreting/ discussing the results of the review? 14. Did the review authors provide a satisfactory explanation for, and discussion of, any heterogeneity observed in the results of the review? 15. If they performed quantitative synthesis did the review authors carry out an adequate investigation of publication bias (small study bias) and discuss its likely impact on the results of the review? 16. Did the review authors report any potential sources of conflict of interest, including any funding they received for conducting the review?

Y, yes; N, no; P, partial yes; M, no meta-analysis conducted.

Methodological Quality of Systematic Reviews using GRADE and Level of Evidence (LOE) using SIGN Methods

\begin{tabular}{ccccccccccc}
\hline $\begin{array}{c}\text { Reference } \\
\text { number }\end{array}$ & $\mathbf{1}$ & $\mathbf{2}$ & $\mathbf{3}$ & $\mathbf{4}$ & $\mathbf{5}$ & $\mathbf{6}$ & $\mathbf{7}$ & $\mathbf{8}$ & GRADE & LOE \\
\hline SR1 & -1 & & -1 & -1 & & & & & Very low (1) & $1-$ \\
\hline
\end{tabular}

1. Limitation, 2. Inconsistency, 3. Indirectness, 4. Imprecision, 5. Publication bias, 6. Large magnitude effect, 7. All plausible confounding would reduce a demonstrated effect or suggest a spurious effect when results show no effect, 8 . Dose response gradient.

The following is the basic searching strategy used for cardiac rehabilitation treatment (KQ 14-18) in the Cochrane Library, combined with the specific searching strategy for each question using AND.

\#1 MeSH descriptor: [Myocardial Ischemia] explode all trees

\#2 ((myocard* or heart* or coronary or cardia*) and (infarct* or isch* or attack*)):ti,ab,kw

\#3 ("acute coronary syndrome" or ACS):ti,ab,kw

\#4 (angina or stenocardia*):ti,ab,kw 


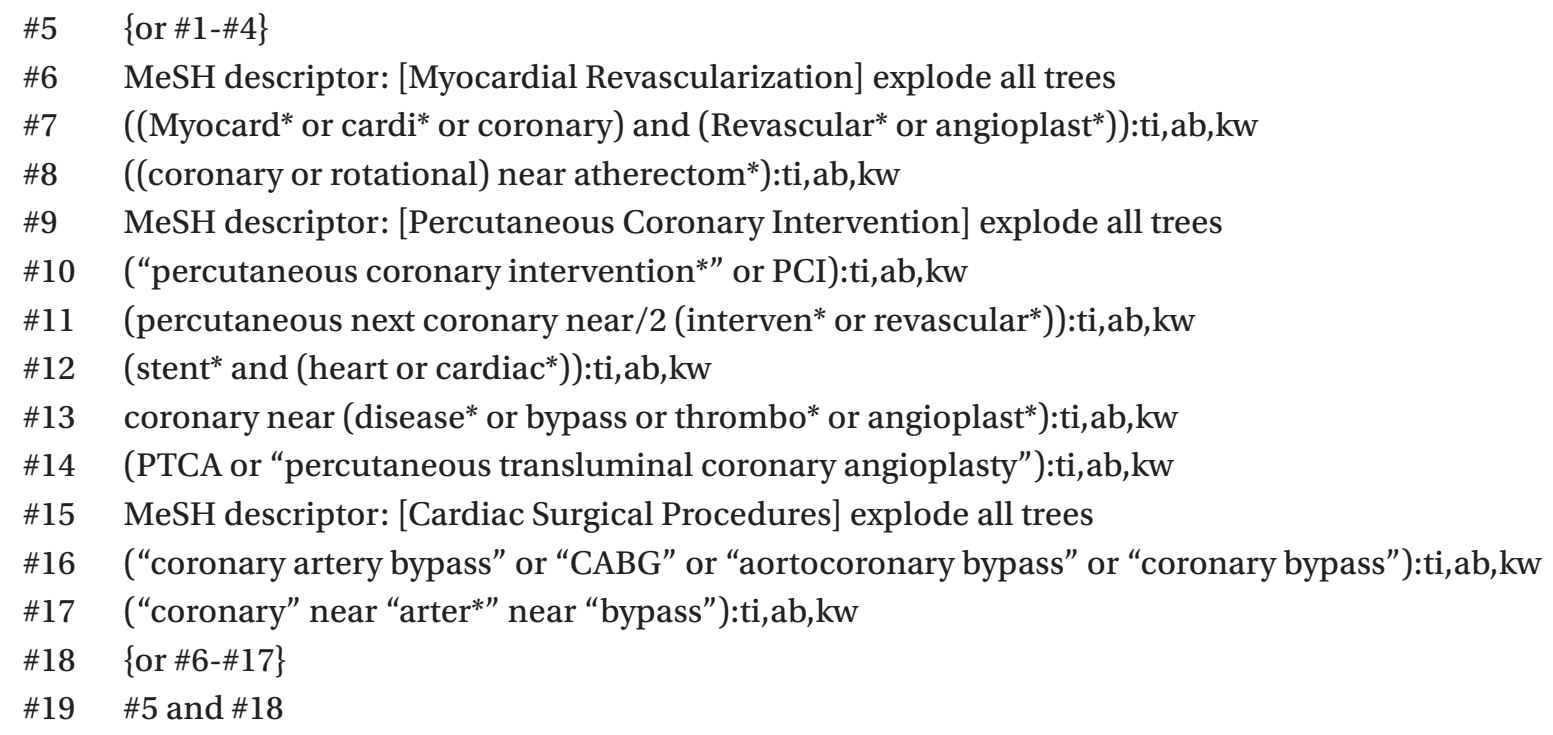

The same Cochrane Library searching strategy was used for KQ14 ("Should aerobic exercise be included in the cardiac rehabilitation program?") and KQ15 ("Should resistance (muscle training) exercise be included in the cardiac rehabilitation program?") The following is the final searching strategy, including the basic searching strategy for exercise therapy for CR:

\section{Searching Strategies}

\#20 MeSH descriptor: [Exercise] explode all trees

\#21 MeSH descriptor: [Exercise Therapy] explode all trees

\#22 (("exercise*" or "train*" or fitness) and ("strength*" or "aerobic")):ti,ab,kw

\#23 (physical near (train* or activ*)):ti,ab,kw

\#24 ((physio or physic* or kinesio*) near therap*):ti,ab,kw

\#25 ((interval or aerobic) near (train* or exercise* or run* or fitness)):ti,ab,kw

\#26 ((muscle* or resistan*) near (train* or activ* or strength* or exercise*)):ti,ab,kw

\#27 \{or \#20-\#26\}

\#28 \#19 and \#27 
Chul Kim, et al.

Flow Chart of Study Selection Process: KQ14

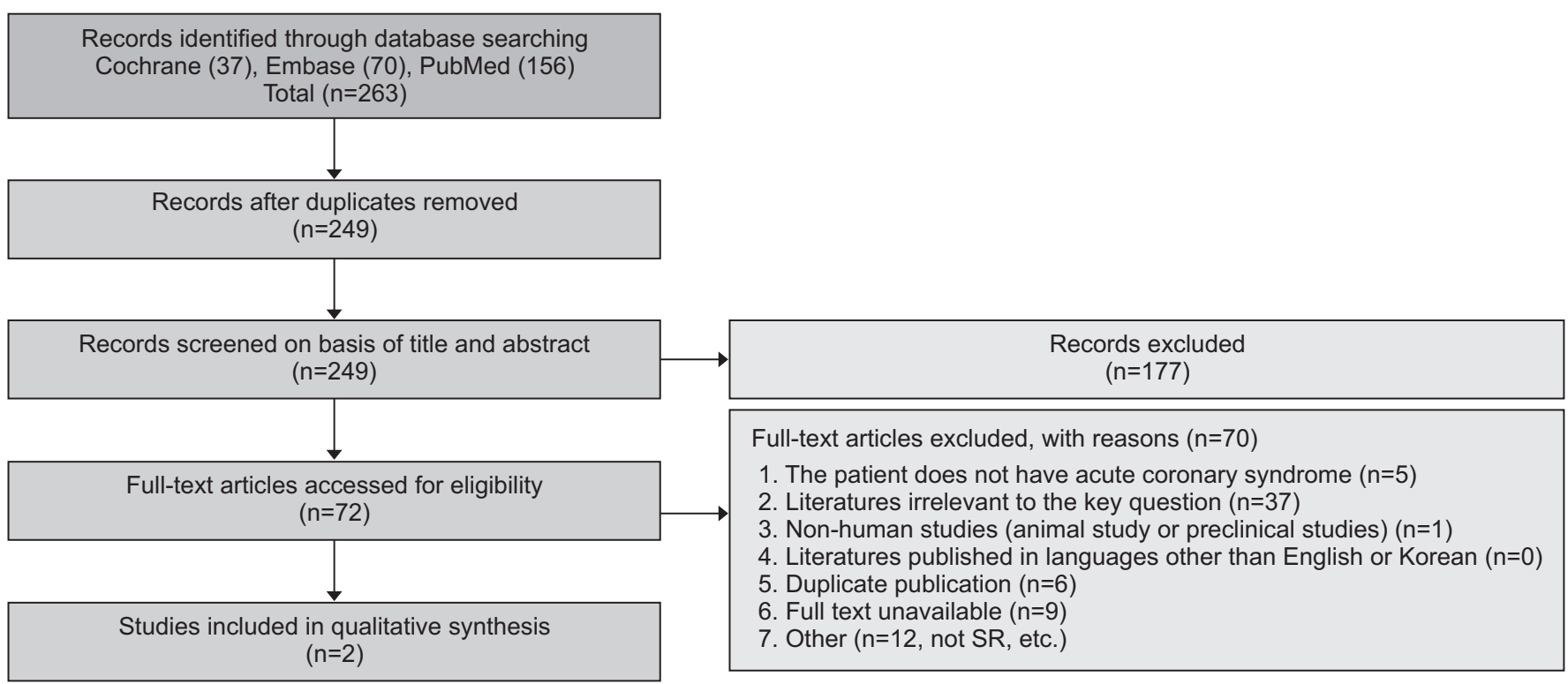

Finally Included Studies: KQ14

\begin{tabular}{cc}
$\begin{array}{c}\text { Reference } \\
\text { number }\end{array}$ & Article \\
\hline SR1 & $\begin{array}{c}\text { Chen YC, Tsai JC, Liou YM, Chan P. Effectiveness of endurance exercise training in patients with coronary } \\
\text { artery disease: a meta-analysis of randomised controlled trials. Eur J Cardiovasc Nurs 2017; 16:397-408. }\end{array}$ \\
SR2 & $\begin{array}{c}\text { Liou K, Ho S, Fildes J, Ooi SY. High intensity interval versus moderate intensity continuous training in } \\
\text { patients with coronary artery disease: a meta-analysis of physiological and clinical parameters. Heart } \\
\text { Lung Circ 2016;25:166-74. }\end{array}$ \\
\hline
\end{tabular}

SR, systematic reviews. 
Evidence Table for Assessment of Risk of Bias and Quality: KQ14

Systematic Reviews (SR)

\begin{tabular}{ccccccccccccccccccc}
\hline $\begin{array}{c}\text { Reference } \\
\text { number }\end{array}$ & $\mathbf{1}$ & $\mathbf{2}$ & $\mathbf{3}$ & $\mathbf{4}$ & $\mathbf{5}$ & $\mathbf{6}$ & $\mathbf{7}$ & $\mathbf{8}$ & $\mathbf{9}$ & $\mathbf{1 0}$ & $\mathbf{1 1}$ & $\mathbf{1 2}$ & $\mathbf{1 3}$ & $\mathbf{1 4}$ & $\mathbf{1 5}$ & $\mathbf{1 6}$ & LOE \\
\hline SR1 & $\mathrm{Y}$ & $\mathrm{Y}$ & $\mathrm{Y}$ & $\mathrm{Y}$ & $\mathrm{Y}$ & $\mathrm{Y}$ & $\mathrm{N}$ & $\mathrm{P}$ & $\mathrm{Y}$ & $\mathrm{N}$ & $\mathrm{Y}$ & $\mathrm{Y}$ & $\mathrm{Y}$ & $\mathrm{Y}$ & $\mathrm{Y}$ & $\mathrm{Y}$ & $1-$ \\
SR2 & $\mathrm{Y}$ & $\mathrm{Y}$ & $\mathrm{Y}$ & $\mathrm{Y}$ & $\mathrm{N}$ & $\mathrm{N}$ & $\mathrm{N}$ & $\mathrm{Y}$ & $\mathrm{Y}$ & $\mathrm{N}$ & $\mathrm{Y}$ & $\mathrm{Y}$ & $\mathrm{Y}$ & $\mathrm{N}$ & $\mathrm{Y}$ & $\mathrm{N}$ & $1+$ \\
\hline
\end{tabular}

1. Did the research questions and inclusion criteria for the review include the components of PICO? 2. Did the report of the review contain an explicit statement that the review methods were established prior to the conduct of the review and did the report justify any significant deviations from the protocol? 3. Did the review authors explain their selection of the study designs for inclusion in the review? 4. Did the review authors use a comprehensive literature search strategy? 5. Did the review authors perform study selection in duplicate? 6. Did the review authors perform data extraction in duplicate? 7. Did the review authors provide a list of excluded studies and justify the exclusions? 8 . Did the review authors describe the included studies in adequate detail? 9. Did the review authors use a satisfactory technique for assessing the risk of bias (RoB) in individual studies that were included in the review? 10. Did the review authors report on the sources of funding for the studies included in the review? 11. If meta-analysis was performed did the review authors use appropriate methods for statistical combination of results? 12. If meta-analysis was performed, did the review authors assess the potential impact of RoB in individual studies on the results of the meta-analysis or other evidence synthesis? 13. Did the review authors account for RoB in individual studies when interpreting/ discussing the results of the review? 14. Did the review authors provide a satisfactory explanation for, and discussion of, any heterogeneity observed in the results of the review? 15. If they performed quantitative synthesis did the review authors carry out an adequate investigation of publication bias (small study bias) and discuss its likely impact on the results of the review? 16. Did the review authors report any potential sources of conflict of interest, including any funding they received for conducting the review?

Y, yes; N, no; P, partial yes.

Methodological Quality of Systematic Reviews using GRADE and Level of Evidence (LOE) using SIGN Methods

\begin{tabular}{cccccccccc}
\hline $\begin{array}{c}\text { Reference } \\
\text { number }\end{array}$ & $\mathbf{1}$ & $\mathbf{2}$ & $\mathbf{3}$ & $\mathbf{4}$ & $\mathbf{5}$ & $\mathbf{6}$ & $\mathbf{7}$ & $\mathbf{8}$ & GRADE \\
\hline SR1 & & -1 & -1 & & & LOE & Very low (1) \\
SR2 & & & -1 & & & & Moderate (3) & $1+$ \\
\hline
\end{tabular}

1. Limitation, 2. Inconsistency, 3. Indirectness, 4. Imprecision, 5. Publication Bias, 6. Large magnitude effect, 7. All plausible confounding would reduce a demonstrated effect or suggest a spurious effect when results show no effect, 8 . Dose response gradient. 


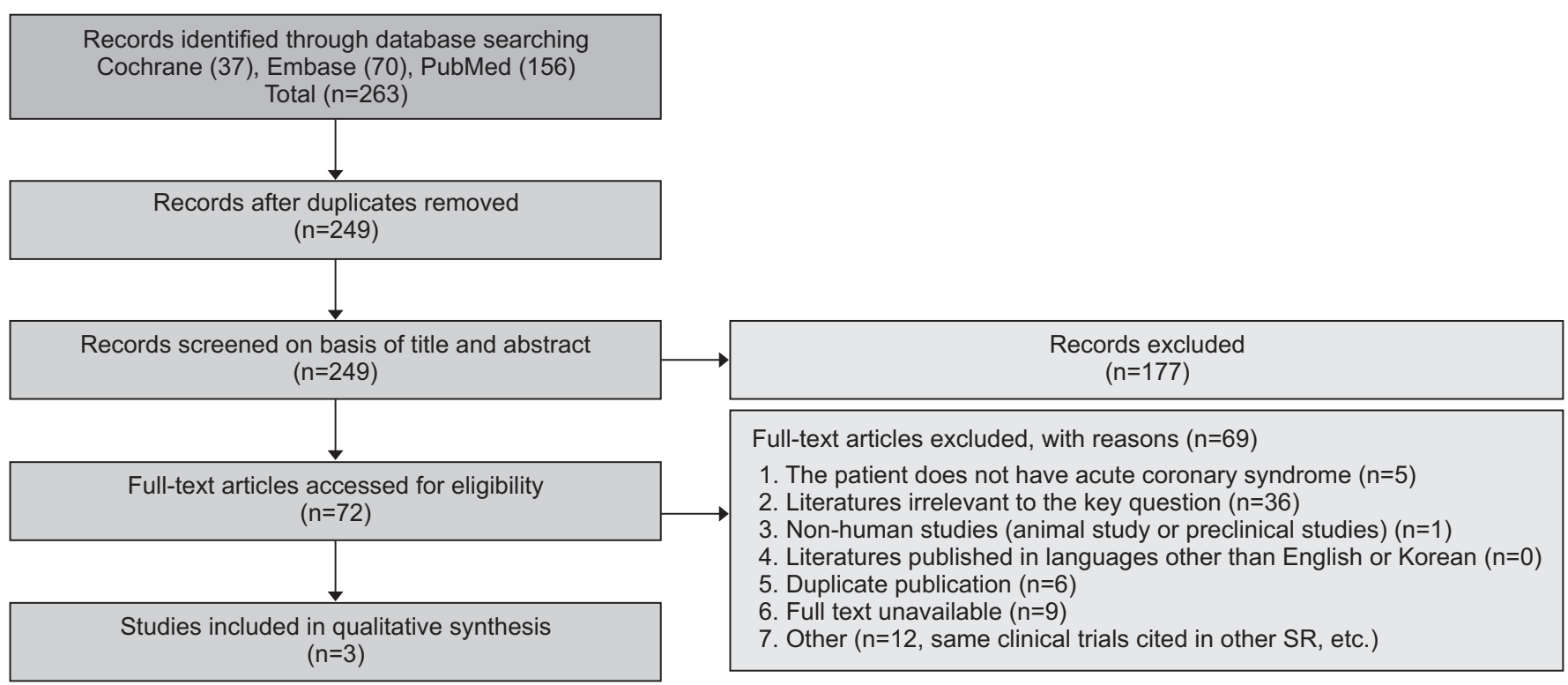

Finally Included Studies: KQ15

\begin{tabular}{cc}
\hline $\begin{array}{c}\text { Reference } \\
\text { number }\end{array}$ & Article \\
\hline SR1 & $\begin{array}{c}\text { Hollings M, Mavros Y, Freeston J, Fiatarone Singh M. The effect of progressive resistance training on aero- } \\
\text { bic fitness and strength in adults with coronary heart disease: a systematic review and meta-analysis of } \\
\text { randomized controlled trials. Eur J Prev Cardiol 2017;24:1242-59. }\end{array}$ \\
SR2 & $\begin{array}{c}\text { Karagiannis C, Savva C, Mamais I, Efstathiou M, Monticone M, Xanthos T. Eccentric exercise in ischemic } \\
\text { cardiac patients and functional capacity: a systematic review and meta-analysis of randomized con- } \\
\text { trolled trials. Ann Phys Rehabil Med 2017;60:58-64. }\end{array}$ \\
SR3 & $\begin{array}{c}\text { Xanthos PD, Gordon BA, Kingsley MI. Implementing resistance training in the rehabilitation of coronary } \\
\text { heart disease: a systematic review and meta-analysis. Int J Cardiol 2017;230:493-508. }\end{array}$ \\
\hline
\end{tabular}

SR, systematic reviews. 
Evidence Table for Assessment of Risk of Bias and Quality: KQ15

Systematic Reviews (SR)

\begin{tabular}{cccccccccccccccccc}
\hline $\begin{array}{c}\text { Reference } \\
\text { number }\end{array}$ & $\mathbf{1}$ & $\mathbf{2}$ & $\mathbf{3}$ & $\mathbf{4}$ & $\mathbf{5}$ & $\mathbf{6}$ & $\mathbf{7}$ & $\mathbf{8}$ & $\mathbf{9}$ & $\mathbf{1 0}$ & $\mathbf{1 1}$ & $\mathbf{1 2}$ & $\mathbf{1 3}$ & $\mathbf{1 4}$ & $\mathbf{1 5}$ & $\mathbf{1 6}$ & LOE \\
\hline SR1 & $\mathrm{Y}$ & $\mathrm{Y}$ & $\mathrm{Y}$ & $\mathrm{Y}$ & $\mathrm{Y}$ & $\mathrm{Y}$ & $\mathrm{Y}$ & $\mathrm{Y}$ & $\mathrm{Y}$ & $\mathrm{N}$ & $\mathrm{Y}$ & $\mathrm{Y}$ & $\mathrm{Y}$ & $\mathrm{Y}$ & $\mathrm{Y}$ & $\mathrm{Y}$ & $1-$ \\
SR2 & $\mathrm{Y}$ & $\mathrm{Y}$ & $\mathrm{Y}$ & $\mathrm{Y}$ & $\mathrm{Y}$ & $\mathrm{Y}$ & $\mathrm{Y}$ & $\mathrm{Y}$ & $\mathrm{Y}$ & $\mathrm{N}$ & $\mathrm{Y}$ & $\mathrm{Y}$ & $\mathrm{Y}$ & $\mathrm{Y}$ & $\mathrm{Y}$ & $\mathrm{Y}$ & $1-$ \\
SR3 & $\mathrm{Y}$ & $\mathrm{Y}$ & $\mathrm{N}$ & $\mathrm{N}$ & $\mathrm{Y}$ & $\mathrm{Y}$ & $\mathrm{Y}$ & $\mathrm{P}$ & $\mathrm{Y}$ & $\mathrm{Y}$ & $\mathrm{Y}$ & $\mathrm{Y}$ & $\mathrm{Y}$ & $\mathrm{Y}$ & $\mathrm{Y}$ & $\mathrm{Y}$ & $1+$ \\
\hline
\end{tabular}

1. Did the research questions and inclusion criteria for the review include the components of PICO? 2. Did the report of the review contain an explicit statement that the review methods were established prior to the conduct of the review and did the report justify any significant deviations from the protocol? 3. Did the review authors explain their selection of the study designs for inclusion in the review? 4. Did the review authors use a comprehensive literature search strategy? 5. Did the review authors perform study selection in duplicate? 6. Did the review authors perform data extraction in duplicate? 7. Did the review authors provide a list of excluded studies and justify the exclusions? 8 . Did the review authors describe the included studies in adequate detail? 9. Did the review authors use a satisfactory technique for assessing the risk of bias (RoB) in individual studies that were included in the review? 10. Did the review authors report on the sources of funding for the studies included in the review? 11. If meta-analysis was performed did the review authors use appropriate methods for statistical combination of results? 12. If meta-analysis was performed, did the review authors assess the potential impact of RoB in individual studies on the results of the meta-analysis or other evidence synthesis? 13. Did the review authors account for RoB in individual studies when interpreting/ discussing the results of the review? 14. Did the review authors provide a satisfactory explanation for, and discussion of, any heterogeneity observed in the results of the review? 15. If they performed quantitative synthesis did the review authors carry out an adequate investigation of publication bias (small study bias) and discuss its likely impact on the results of the review? 16. Did the review authors report any potential sources of conflict of interest, including any funding they received for conducting the review?

Y, yes; N, no; P, partial yes.

Methodological Quality of Systematic Reviews using GRADE and Level of Evidence (LOE) using SIGN Methods

\begin{tabular}{ccccccccccc}
\hline $\begin{array}{c}\text { Reference } \\
\text { number }\end{array}$ & $\mathbf{1}$ & $\mathbf{2}$ & $\mathbf{3}$ & $\mathbf{4}$ & $\mathbf{5}$ & $\mathbf{6}$ & $\mathbf{7}$ & $\mathbf{8}$ & GRADE & LOE \\
\hline SR1 & & & -2 & -1 & -1 & +1 & & Very low (1) & $1-$ \\
SR2 & -1 & & & -1 & -1 & & & & Very low (1) & $1-$ \\
SR3 & & & & -1 & & & & & Moderate (3) & $1+$ \\
\hline
\end{tabular}

1. Limitation, 2. Inconsistency, 3. Indirectness, 4. Imprecision, 5. Publication Bias, 6. Large magnitude effect, 7. All plausible confounding would reduce a demonstrated effect or suggest a spurious effect when results show no effect, 8 . Dose response gradient.

The following is the final Cochrane Library searching strategy used for KQ16 ("How can the safety of cardiac rehabilitation exercise be enhanced?") including the basic searching strategy for exercise therapy for CR:

\section{Searching Strategies}

\#20 MeSH descriptor: [Cardiac Rehabilitation] explode all trees

\#21 (( ${ }^{*}$ cardia* $^{*}$ or heart* $)$ and *habilitation*):ti,ab,kw

\#22 \{or \#20-\#21\}

\#23 MeSH descriptor: [Wireless Technology] explode all trees

\#24 MeSH descriptor: [Electrocardiography] explode all trees

\#25 ((electrocardiography or ecg or ekg) near monitor*):ti,ab,kw 
Chul Kim, et al.

\#26 MeSH descriptor: [Safety] explode all trees

\#27 (safe* or risk):ti,ab,kw

\#28 \{or \#23-\#27\}

\#29 \#19 and \#22 and \#28

Flow Chart of Study Selection Process

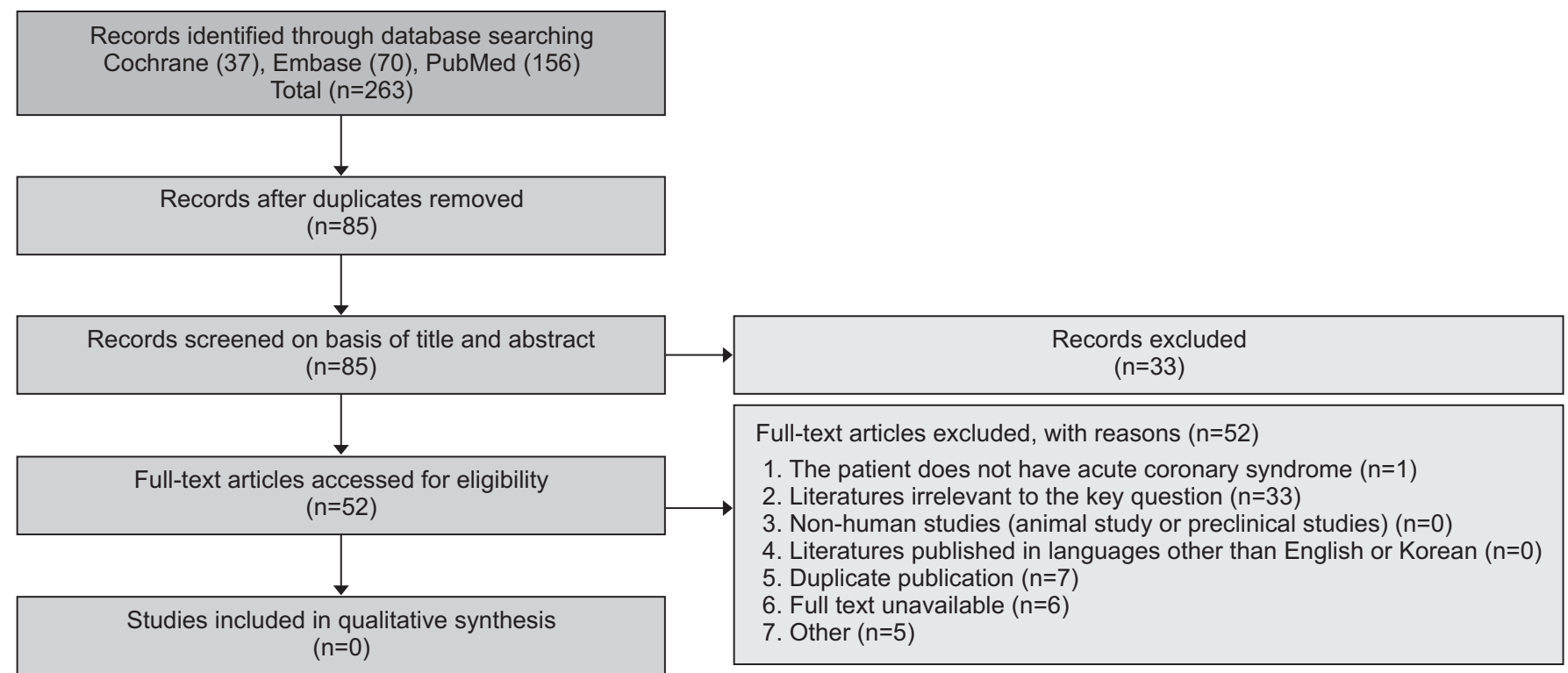

Finally Included Studies

Reference number

Article

The following is the final Cochrane Library searching strategy used for KQ17 ("Can a home-based cardiac rehabilitation program replace a hospital-based cardiac rehabilitation program?") including the basic searching strategy for exercise therapy for $\mathrm{CR}$ :

\section{Searching Strategies}

\#20 MeSH descriptor: [Cardiac Rehabilitation] explode all trees

\#21 ((*ardia* or heart*) and *habilitation*):ti, ab,kw

\#22 MeSH descriptor: [Rehabilitation] explode all trees

\#23 (rehabilitat* or *habilitation):ti, ab,kw

\#24 MeSH descriptor: [Physical and Rehabilitation Medicine] explode all trees

\#25 MeSH descriptor: [Health Facilities] explode all trees

\#26 MeSH descriptor: [Rehabilitation Nursing] explode all trees

\#27 \{or \#20-\#26\}

\#28 (home or center or centre):ti,ab,kw

\#29 \#19 and \#27 and \#28 
Flow Chart of Study Selection Process

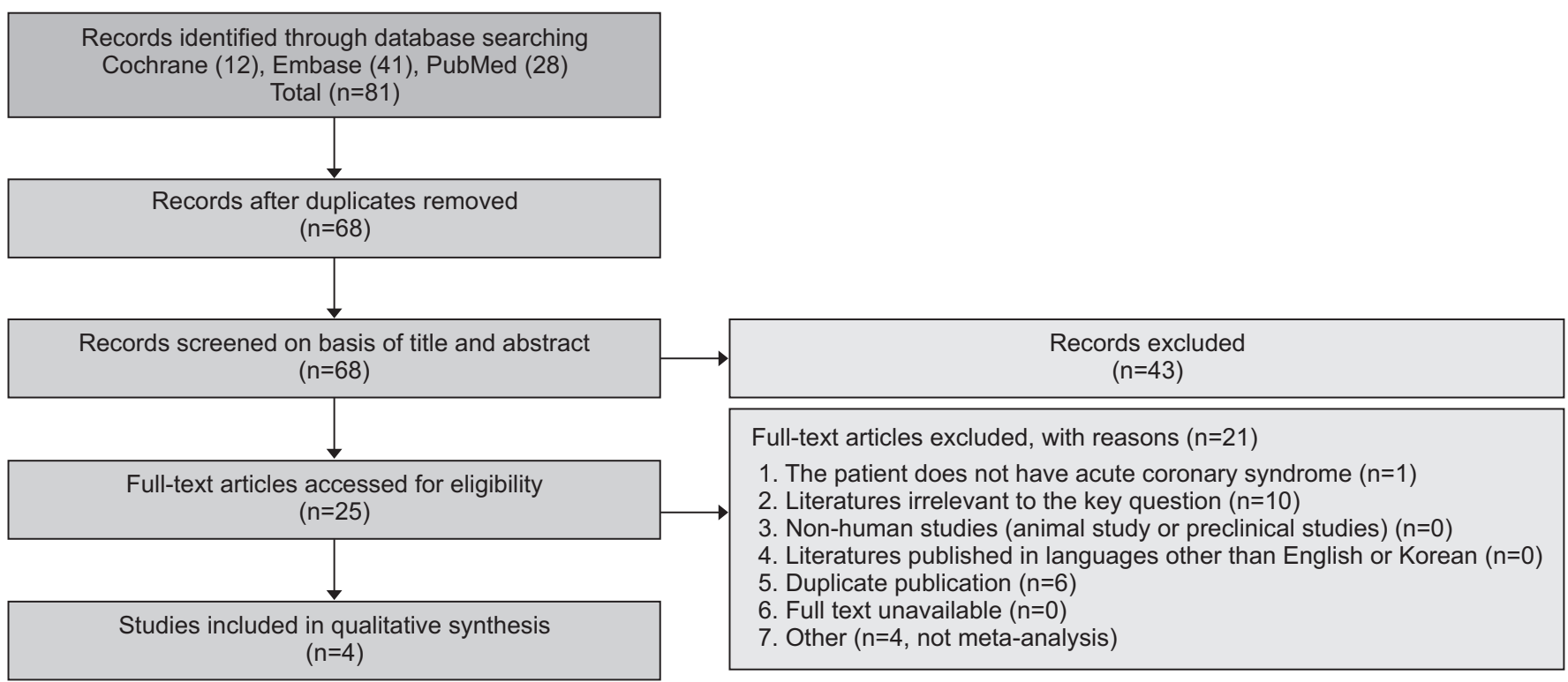

Finally Included Studies

\begin{tabular}{cc}
\hline $\begin{array}{c}\text { Reference } \\
\text { number }\end{array}$ & Article \\
\hline SR1 & $\begin{array}{c}\text { Claes J, Buys R, Budts W, Smart N, Cornelissen VA. Longer-term effects of home-based exercise interven- } \\
\text { tions on exercise capacity and physical activity in coronary artery disease patients: a systematic review } \\
\text { and meta-analysis. Eur J Prev Cardiol 2017;24:244-56. }\end{array}$ \\
SR2 & $\begin{array}{c}\text { Anderson L, Sharp GA, Norton RJ, Dalal H, Dean SG, Jolly K, et al. Home-based versus centre-based car- } \\
\text { diac rehabilitation. Cochrane Database Syst Rev 2017;6:CD007130. }\end{array}$ \\
SR3 & $\begin{array}{c}\text { Huang K, Liu W, He D, Huang B, Xiao D, Peng Y, et al. Telehealth interventions versus center-based car- } \\
\text { diac rehabilitation of coronary artery disease: a systematic review and meta-analysis. Eur J Prev Cardiol }\end{array}$ \\
& $\begin{array}{c}\text { 2015;22:959-71. } \\
\text { ScClure T, Haykowsky MJ, Schopflocher D, Hsu ZY, Clark AM. Home-based secondary prevention pro- } \\
\text { grams for patients with coronary artery disease: a meta-analysis of effects on anxiety. J Cardiopulm Re- } \\
\text { habil Prev 2013;33:59-67. }\end{array}$ \\
\hline
\end{tabular}

SR, systematic reviews. 
Evidence Table for Assessment of Risk of Bias and Quality: KQ17

\begin{tabular}{ccccccccccccccccccc}
\hline $\begin{array}{c}\text { Reference } \\
\text { number }\end{array}$ & $\mathbf{1 1}$ & $\mathbf{2}$ & $\mathbf{3}$ & $\mathbf{4}$ & $\mathbf{5}$ & $\mathbf{6}$ & $\mathbf{7}$ & $\mathbf{8}$ & $\mathbf{9}$ & $\mathbf{1 0}$ & $\mathbf{1 1}$ & $\mathbf{1 2}$ & $\mathbf{1 3}$ & $\mathbf{1 4}$ & $\mathbf{1 5}$ & $\mathbf{1 6}$ & LOE \\
\hline SR1 & $\mathrm{Y}$ & $\mathrm{Y}$ & $\mathrm{Y}$ & $\mathrm{Y}$ & $\mathrm{Y}$ & $\mathrm{Y}$ & $\mathrm{Y}$ & $\mathrm{Y}$ & $\mathrm{Y}$ & $\mathrm{Y}$ & $\mathrm{Y}$ & $\mathrm{Y}$ & $\mathrm{Y}$ & $\mathrm{Y}$ & $\mathrm{Y}$ & $\mathrm{Y}$ & $1-$ \\
SR2 & $\mathrm{Y}$ & $\mathrm{Y}$ & $\mathrm{Y}$ & $\mathrm{Y}$ & $\mathrm{Y}$ & $\mathrm{Y}$ & $\mathrm{Y}$ & $\mathrm{Y}$ & $\mathrm{Y}$ & $\mathrm{N}$ & $\mathrm{N}$ & $\mathrm{N}$ & $\mathrm{N}$ & $\mathrm{N}$ & $\mathrm{N}$ & $\mathrm{N}$ & $1+$ \\
SR3 & $\mathrm{Y}$ & $\mathrm{Y}$ & $\mathrm{Y}$ & $\mathrm{Y}$ & $\mathrm{Y}$ & $\mathrm{Y}$ & $\mathrm{Y}$ & $\mathrm{Y}$ & $\mathrm{Y}$ & $\mathrm{N}$ & $\mathrm{Y}$ & $\mathrm{Y}$ & $\mathrm{Y}$ & $\mathrm{Y}$ & $\mathrm{Y}$ & $\mathrm{Y}$ & $1-$ \\
SR4 & $\mathrm{Y}$ & $\mathrm{Y}$ & $\mathrm{Y}$ & $\mathrm{P}$ & $\mathrm{Y}$ & $\mathrm{P}$ & $\mathrm{P}$ & $\mathrm{Y}$ & $\mathrm{Y}$ & $\mathrm{N}$ & $\mathrm{Y}$ & $\mathrm{Y}$ & $\mathrm{Y}$ & $\mathrm{Y}$ & $\mathrm{Y}$ & $\mathrm{N}$ & $1-$ \\
\hline
\end{tabular}

1. Did the research questions and inclusion criteria for the review include the components of PICO? 2. Did the report of the review contain an explicit statement that the review methods were established prior to the conduct of the review and did the report justify any significant deviations from the protocol? 3. Did the review authors explain their selection of the study designs for inclusion in the review? 4. Did the review authors use a comprehensive literature search strategy? 5. Did the review authors perform study selection in duplicate? 6. Did the review authors perform data extraction in duplicate? 7. Did the review authors provide a list of excluded studies and justify the exclusions? 8. Did the review authors describe the included studies in adequate detail? 9. Did the review authors use a satisfactory technique for assessing the risk of bias (RoB) in individual studies that were included in the review? 10. Did the review authors report on the sources of funding for the studies included in the review? 11. If meta-analysis was performed did the review authors use appropriate methods for statistical combination of results? 12. If meta-analysis was performed, did the review authors assess the potential impact of RoB in individual studies on the results of the meta-analysis or other evidence synthesis? 13. Did the review authors account for RoB in individual studies when interpreting/ discussing the results of the review? 14. Did the review authors provide a satisfactory explanation for, and discussion of, any heterogeneity observed in the results of the review? 15. If they performed quantitative synthesis did the review authors carry out an adequate investigation of publication bias (small study bias) and discuss its likely impact on the results of the review? 16. Did the review authors report any potential sources of conflict of interest, including any funding they received for conducting the review?

$\mathrm{Y}$, yes; N, no; P, partial yes.

Methodological Quality of Systematic Reviews using GRADE and Level of Evidence (LOE) using SIGN Methods

\begin{tabular}{ccccccccccc}
\hline $\begin{array}{c}\text { Reference } \\
\text { number }\end{array}$ & $\mathbf{1}$ & $\mathbf{2}$ & $\mathbf{3}$ & $\mathbf{4}$ & $\mathbf{5}$ & $\mathbf{6}$ & $\mathbf{7}$ & $\mathbf{8}$ & GRADE & LOE \\
\hline SR1 & -1 & & -1 & & & & & & Low (2) & $1-$ \\
SR2 & & & -1 & -1 & & +1 & & & Moderate (3) & $1+$ \\
SR3 & -1 & & -1 & & & & & Low (2) & $1-$ \\
SR4 & -1 & & -1 & & -1 & & & & Very low (1) & $1-$ \\
\hline
\end{tabular}

1. Limitation, 2. Inconsistency, 3. Indirectness, 4. Imprecision, 5. Publication Bias, 6. Large magnitude effect, 7. All plausible confounding would reduce a demonstrated effect or suggest a spurious effect when results show no effect, 8 . Dose response gradient.

The following is the final Cochrane Library searching strategy used for KQ18 ("Should cardiac rehabilitation programs be recommended to elderly patients?") including the basic searching strategy for exercise therapy for CR:

\section{Searching Strategies}

MeSH descriptor: [Cardiac Rehabilitation] explode all trees

\#21 ((*ardia* or heart*) and *habilitation*):ti,ab,kw

\#22 MeSH descriptor: [Rehabilitation] explode all trees

\#23 (rehabilitat* or *habilitation):ti,ab,kw

\#24 MeSH descriptor: [Physical and Rehabilitation Medicine] explode all trees

\#25 MeSH descriptor: [Health Facilities] explode all trees 
\#26 MeSH descriptor: [Rehabilitation Nursing] explode all trees

\#27 \{or \#20-\#26\}

\#28 MeSH descriptor: [Women] explode all trees

\#29 MeSH descriptor: [Aged] explode all trees

\#30 (women or woman or female or gender):ti,ab,kw

\#31 (old* or elder* or aged):ti,ab,kw

\#32 \{or \#28-\#31\}

\#33 \#19 and \#27 and \#32

Flow Chart of Study Selection Process

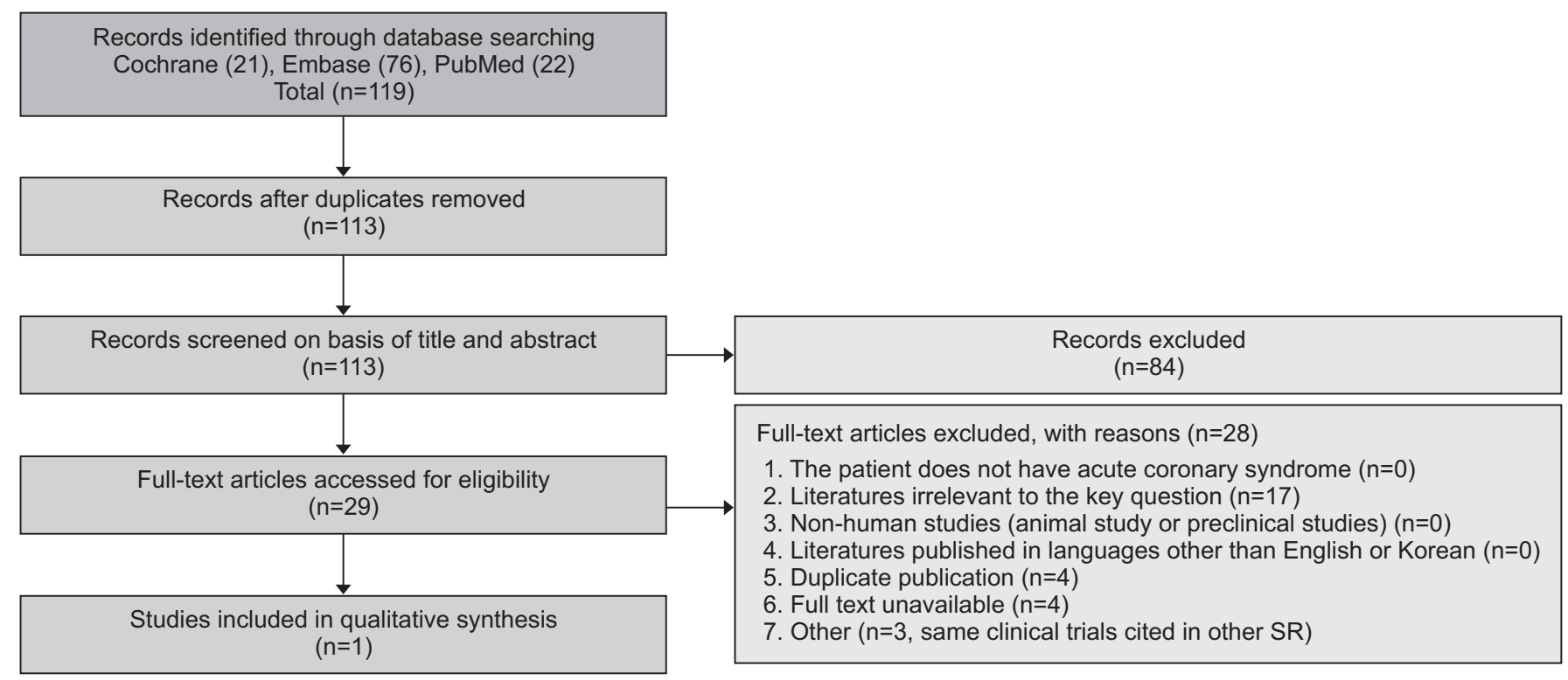

Finally Included Studies

\begin{tabular}{cl}
\hline $\begin{array}{c}\text { Reference } \\
\text { number }\end{array}$ & \multicolumn{1}{c}{ Article } \\
\hline SR1 & $\begin{array}{l}\text { Yamamoto S, Hotta K, Ota E, Mori R, Matsunaga A. Effects of resistance training on muscle strength, ex- } \\
\text { ercise capacity, and mobility in middle-aged and elderly patients with coronary artery disease: a meta- } \\
\text { analysis. J Cardiol 2016;68:125-34. }\end{array}$ \\
\hline
\end{tabular}

SR, systematic reviews. 
Evidence Table for Assessment of Risk of Bias and Quality

Methodological Quality of Systematic Reviews using AMSTAR 2.0 and Level of Evidence (LOE) using SIGN Methods

\begin{tabular}{cccccccccccccccccc}
\hline $\begin{array}{c}\text { Reference } \\
\text { number }\end{array}$ & $\mathbf{1}$ & $\mathbf{2}$ & $\mathbf{3}$ & $\mathbf{4}$ & $\mathbf{5}$ & $\mathbf{6}$ & $\mathbf{7}$ & $\mathbf{8}$ & $\mathbf{9}$ & $\mathbf{1 0}$ & $\mathbf{1 1}$ & $\mathbf{1 2}$ & $\mathbf{1 3}$ & $\mathbf{1 4}$ & $\mathbf{1 5}$ & $\mathbf{1 6}$ & \multirow{2}{*}{ LOE } \\
\hline $\mathrm{SR} 1$ & $\mathrm{Y}$ & $\mathrm{Y}$ & $\mathrm{Y}$ & $\mathrm{Y}$ & $\mathrm{Y}$ & $\mathrm{Y}$ & $\mathrm{P}$ & $\mathrm{Y}$ & $\mathrm{Y}$ & $\mathrm{N}$ & $\mathrm{Y}$ & $\mathrm{Y}$ & $\mathrm{Y}$ & $\mathrm{Y}$ & $\mathrm{Y}$ & $\mathrm{Y}$ & $1++$ \\
\hline
\end{tabular}

1. Did the research questions and inclusion criteria for the review include the components of PICO? 2. Did the report of the review contain an explicit statement that the review methods were established prior to the conduct of the review and did the report justify any significant deviations from the protocol? 3. Did the review authors explain their selection of the study designs for inclusion in the review? 4. Did the review authors use a comprehensive literature search strategy? 5. Did the review authors perform study selection in duplicate? 6. Did the review authors perform data extraction in duplicate? 7. Did the review authors provide a list of excluded studies and justify the exclusions? 8 . Did the review authors describe the included studies in adequate detail? 9. Did the review authors use a satisfactory technique for assessing the risk of bias (RoB) in individual studies that were included in the review? 10. Did the review authors report on the sources of funding for the studies included in the review? 11. If meta-analysis was performed did the review authors use appropriate methods for statistical combination of results? 12. If meta-analysis was performed, did the review authors assess the potential impact of RoB in individual studies on the results of the meta-analysis or other evidence synthesis? 13. Did the review authors account for RoB in individual studies when interpreting/ discussing the results of the review? 14. Did the review authors provide a satisfactory explanation for, and discussion of, any heterogeneity observed in the results of the review? 15. If they performed quantitative synthesis did the review authors carry out an adequate investigation of publication bias (small study bias) and discuss its likely impact on the results of the review? 16. Did the review authors report any potential sources of conflict of interest, including any funding they received for conducting the review?

$\mathrm{Y}$, yes; N, no; P, partial yes.

Methodological Quality of Systematic Reviews using GRADE and Level of Evidence (LOE) using SIGN Methods

\begin{tabular}{cccccccccccc}
\hline $\begin{array}{c}\text { Reference } \\
\text { number }\end{array}$ & 1 & 2 & 3 & 4 & 5 & 6 & 7 & 8 & GRADE & LOE \\
\hline SR1 & & & & & & & & & & High $(4)$ & $1++$ \\
\hline
\end{tabular}

1. Limitation, 2. Inconsistency, 3. Indirectness, 4. Imprecision, 5. Publication Bias, 6. Large magnitude effect, 7. All plausible confounding would reduce a demonstrated effect or suggest a spurious effect when results show no effect, 8 . Dose response gradient.

\section{EDUCATION FOR CARDIAC REHABILITATION}

The following is the basic searching strategy commonly used in education for cardiac rehabilitation in the Cochrane Library. This basic searching strategy was combined with the specific searching strategy for each question using AND.

MeSH descriptor: [Myocardial Ischemia] explode all trees

\#17 ((myocard* or heart* or coronary or cardia*) and (infarct* or isch* or attack*)):ti,ab,kw

\#18 ("acute coronary syndrome" or ACS):ti,ab,kw

\#19 (angina or stenocardia*):ti,ab,kw

\#20 MeSH descriptor: [Myocardial Revascularization] explode all trees

\#21 ((Myocard* or cardi* or coronary) and (Revascular* or angioplast*)):ti,ab,kw

\#22 ((coronary or rotational) near atherectom*):ti,ab,kw

\#23 ("coronary artery bypass" or CABG or "aortocoronary bypass" or "coronary bypass"):ti, ab,kw

\#24 MeSH descriptor: [Percutaneous Coronary Intervention] explode all trees

\#25 ("percutaneous coronary intervention*" or PCI):ti,ab,kw 


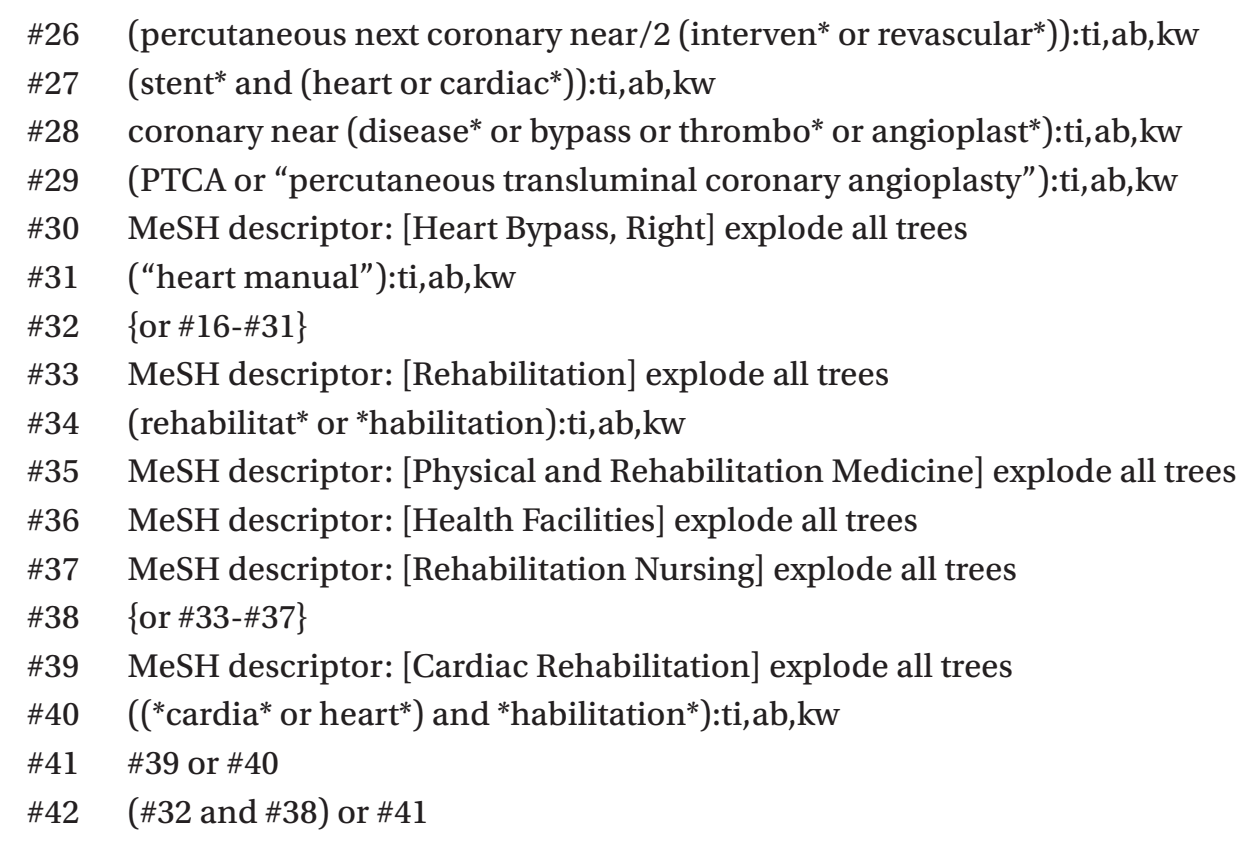

The following is the final Cochrane Library searching strategy used for KQ19 ("Is patient education necessary as part of cardiac rehabilitation?") including the basic searching strategy for education for secondary prevention:

\section{Searching Strategies}

\#1 MeSH descriptor: [Health Behavior] explode all trees

\#2 (health next behaviour* or health next behavior*):ti,ab,kw

\#3 MeSH descriptor: [Patient Discharge] explode all trees

\#4 ("patient discharge" or discharging next patient*):ti,ab,kw

\#5 MeSH descriptor: [Patient Transfer] explode all trees

\#6 MeSH descriptor: [Patient Handoff] explode all trees

\#7 (handoff or handover or "hand off" or "hand over"):ti,ab,kw

\#8 shared care:ti,ab,kw

\#9 ("behaviour change" or "behavior change"):ti,ab,kw

\#10 (maintenance*):ti,ab,kw

\#11 (transition* near/3 care):ti,ab,kw

\#12 ("peer support" or "peer mentoring" or peer next counsel*):ti,ab,kw

\#13 MeSH descriptor: [Health Education] explode all trees

\#14 ("health education"):ti,ab,kw

\#15 \{or \#1-\#14\} 


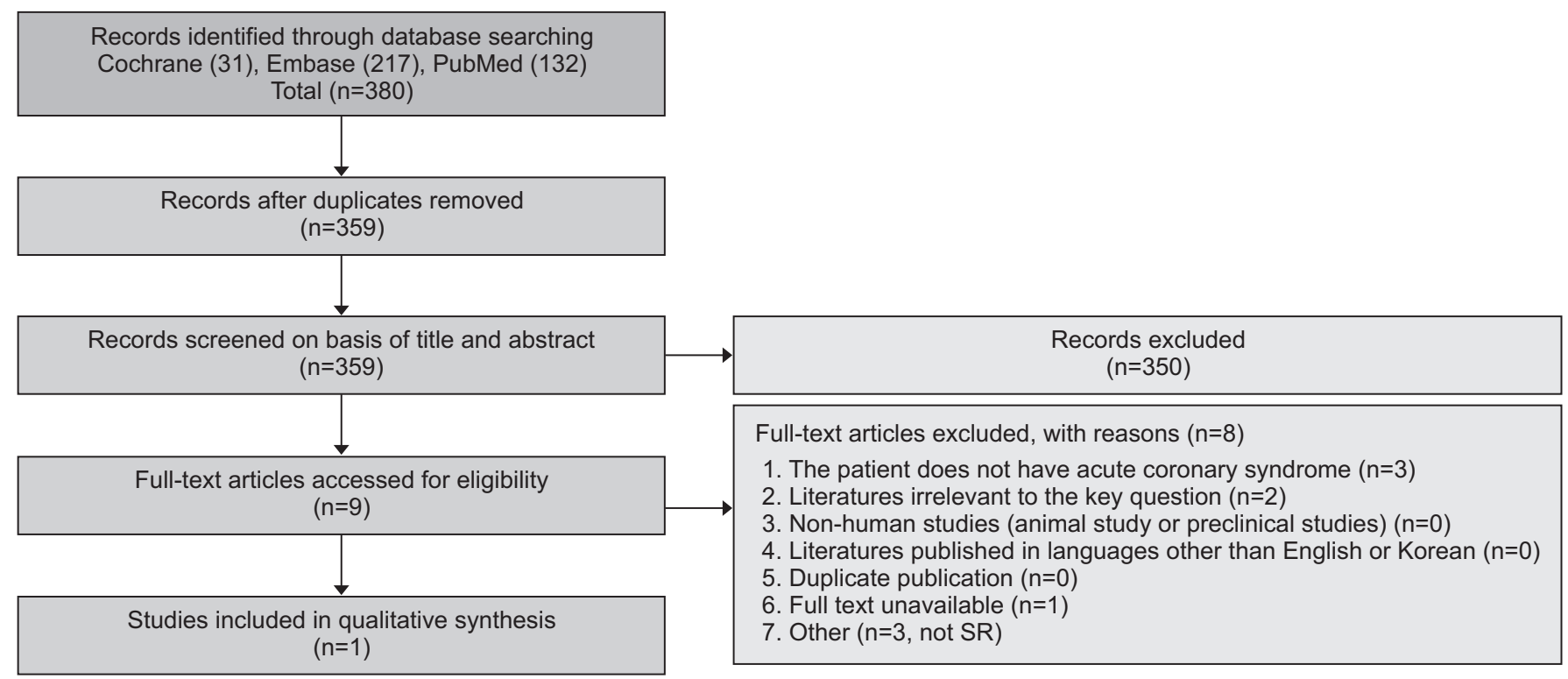

Finally Included Studies

\begin{tabular}{cc}
$\begin{array}{c}\text { Reference } \\
\text { Number }\end{array}$ & Article \\
\hline SR1 & $\begin{array}{c}\text { Anderson L, Brown JP, Clark AM, Dalal H, Rossau HK, Bridges C, et al. Patient education in the manage- } \\
\text { ment of coronary heart disease. Cochrane Database Syst Rev 2017;6:CD008895. }\end{array}$ \\
\hline
\end{tabular}

SR, systematic reviews.

Evidence Table for Assessment of Risk of Bias and Quality

Methodological Quality of Systematic Reviews using GRADE and Level of Evidence (LOE) using SIGN Methods

\begin{tabular}{|c|c|c|c|c|c|c|c|c|c|c|}
\hline $\begin{array}{c}\text { Reference } \\
\text { number }\end{array}$ & 1 & 2 & 3 & 4 & 5 & 6 & 7 & 8 & GRADE & LOE \\
\hline SRl & & & 1 & 1 & & & & & Low (2) & $1-$ \\
\hline
\end{tabular}

1. Limitation, 2. Inconsistency, 3. Indirectness, 4. Imprecision, 5. Publication Bias, 6. Large magnitude effect, 7. All plausible confounding would reduce a demonstrated effect or suggest a spurious effect when results show no effect, 8. Dose response gradient.

The following is the final Cochrane Library searching strategy used for KQ20 ("What contents should be included in patient education?") and KQ21 ("What interventions are needed to boost patients' drug compliance?") including the basic searching strategy in education for cardiac rehabilitation:

\section{Searching Strategies}

\#1 Medication Adherence*:ti,ab,kw

\#2 medication adj3 (adherence* or "non-adherence" or nonadherence* or "non adherence" or compliance* or non-compliance* or "non compliance" or persistence*):ti,ab,kw

\#3 *Patient Compliance:ti, ab,kw

\#4 MeSH descriptor: [Medication Adherence] explode all trees

\#5 \#1 or \#2 or \#3 or \#4 
Flow Chart of Study Selection Process

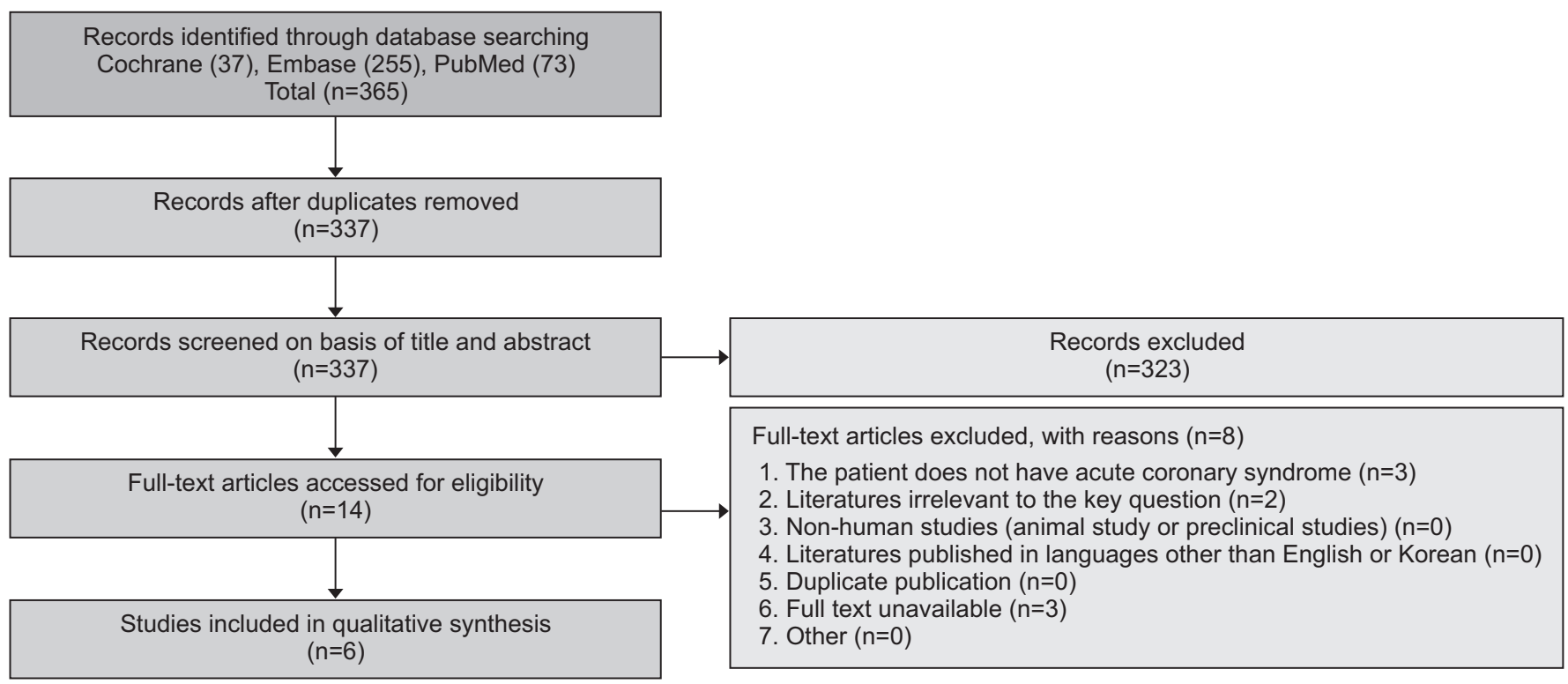

Finally Included Studies

\begin{tabular}{cc}
$\begin{array}{c}\text { Reference } \\
\text { number }\end{array}$ & Article \\
\hline SR1 & $\begin{array}{c}\text { Adler AJ, Martin N, Mariani J, Tajer CD, Owolabi OO, Free C, et al. Mobile phone text messaging to im- } \\
\text { prove medication adherence in secondary prevention of cardiovascular disease. Cochrane Database }\end{array}$ \\
Syst Rev 2017;4:CD011851.
\end{tabular}

SR, systematic reviews. 
Chul Kim, et al.

Evidence Table for Assessment of Risk of Bias and Quality

Methodological Quality of Systematic Reviews using GRADE and Level of Evidence (LOE) using SIGN Methods

\begin{tabular}{|c|c|c|c|c|c|c|c|c|c|c|}
\hline $\begin{array}{c}\text { Reference } \\
\text { number }\end{array}$ & 1 & 2 & 3 & 4 & 5 & 6 & 7 & 8 & GRADE & LOE \\
\hline SR1 & & & 1 & 1 & & & & & Low (2) & $1-$ \\
\hline SR2 & 1 & & & 1 & 1 & & & & Very low (1) & $1-$ \\
\hline SR3 & 1 & & 1 & 1 & & & & & Very low (1) & $1-$ \\
\hline SR4 & 1 & & 1 & 1 & & & & & Very low (1) & $1-$ \\
\hline SR5 & 1 & & & 1 & & & & & Low (2) & $1-$ \\
\hline SR6 & 1 & & 1 & & & & & & Low (2) & $1-$ \\
\hline
\end{tabular}

1. Limitation, 2. Inconsistency, 3. Indirectness, 4. Imprecision, 5. Publication Bias, 6. Large magnitude effect, 7. All plausible confounding would reduce a demonstrated effect or suggest a spurious effect when results show no effect, 8. Dose response gradient.

The following is the final Cochrane Library searching strategy used for KQ22 ("What is a good brief intervention for patients who need to quit smoking?") including the basic searching strategy for education for cardiac rehabilitation:

\section{Searching Strategies}

\#1 brief smoking cessation:ti,ab,kw

\#2 (brief intervention* near/4 smoking):ti,ab,kw

\#3 (brief near/3 smoking cessation):ti,ab,kw

\#4 \#1 or \#2 or \#3

\#5 MeSH descriptor: [Tobacco Use Cessation] explode all trees

\#6 MeSH descriptor: [Tobacco Use] explode all trees

\#7 ((smok* or tobacco or cigar* or nicotine) near/3 (quit* or stop* or ceas* or cessation)):ti,ab,kw

\#8 MeSH descriptor: [Tobacco Use Disorder] explode all trees

\#9 MeSH descriptor: [Tobacco Products] explode all trees

\#10 \#6 or \#8 or \#9

\#11 (quit* or stop* or ceas* or cessation):ti,ab,kw

\#12 \#10 and \#11

\#13 \#5 or \# 7 or \#12 
Flow Chart of Study Selection Process

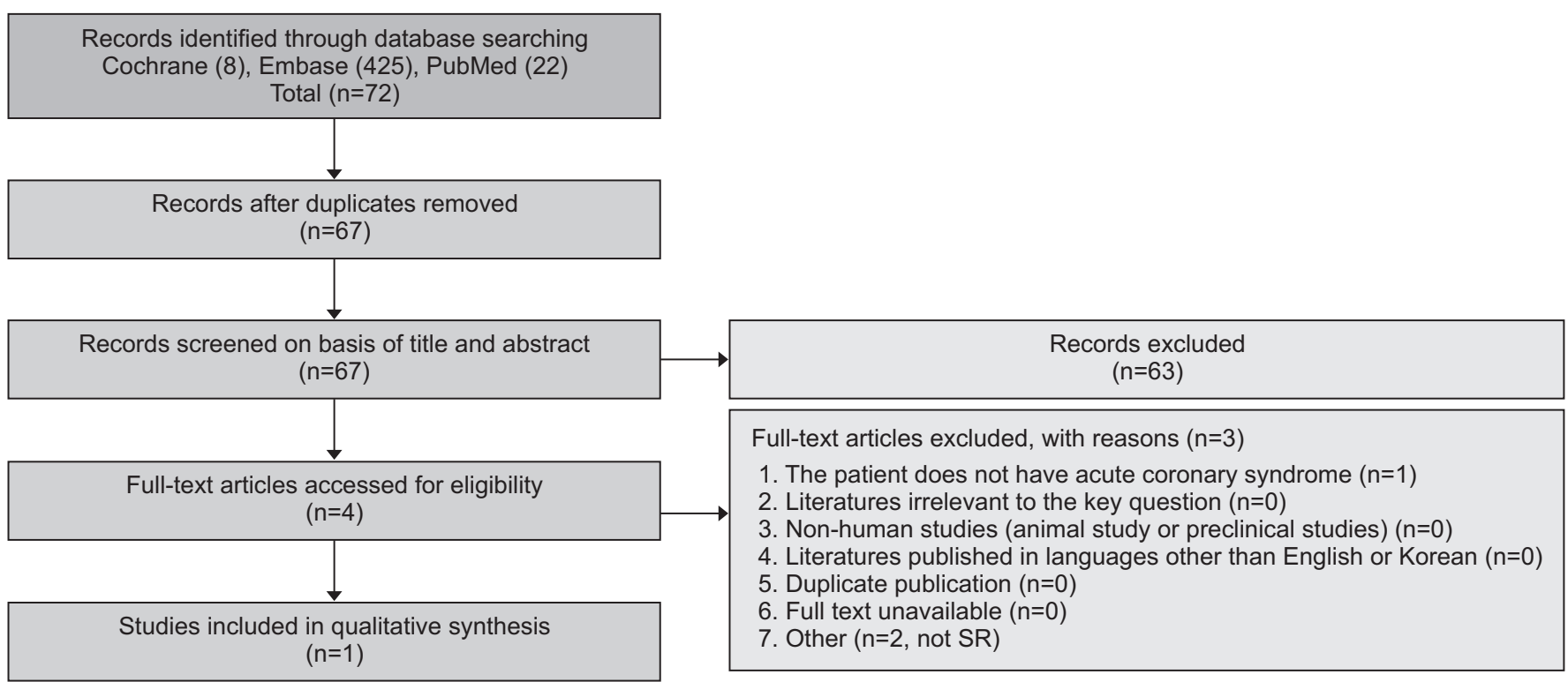

Finally Included Studies

\begin{tabular}{cc}
$\begin{array}{c}\text { Reference } \\
\text { number }\end{array}$ & Article \\
\hline SR1 & $\begin{array}{c}\text { Franck C, Filion KB, Eisenberg MJ. Smoking cessation in patients with acute coronary syndrome. Am J } \\
\text { Cardiol 2018;121:1105-11. }\end{array}$ \\
\hline
\end{tabular}

SR, systematic reviews.

Evidence Table for Assessment of Risk of Bias and Quality

Methodological Quality of Systematic Reviews using GRADE and Level of Evidence (LOE) using SIGN Methods

\begin{tabular}{ccccccccccc}
\hline $\begin{array}{c}\text { Reference } \\
\text { Number }\end{array}$ & $\mathbf{1}$ & $\mathbf{2}$ & $\mathbf{3}$ & $\mathbf{4}$ & $\mathbf{5}$ & $\mathbf{6}$ & $\mathbf{7}$ & $\mathbf{8}$ & GRADE & LOE \\
\hline SR1 & 1 & & 1 & 1 & & & & Very low (1) & $1-$ \\
\hline
\end{tabular}

1. Limitation, 2. Inconsistency, 3. Indirectness, 4. Imprecision, 5. Publication Bias, 6. Large magnitude effect, 7. All plausible confounding would reduce a demonstrated effect or suggest a spurious effect when results show no effect, 8 . Dose response gradient.

KQ23. Should a specific food supplement be recommended for patients undergoing cardiac rehabilitation? For KQ24-1, omega-3: The following searching strategy was used without combining the common searching strategy:

\section{Searching Strategies}

\#1 (omega-3 or "omega 3") .ti,ab.

\#2 MeSH descriptor: [Fatty Acids, Omega-3] explode all trees

\#3 \#1 or \#2

\#4 MeSH descriptor: [Myocardial Ischemia] explode all trees

\#5 ((myocard* or heart* or coronary or cardia*) and (infarct* or isch* or attack*)):ti,ab,kw 
\#6 ("acute coronary syndrome" or ACS):ti,ab,kw

\#7 (angina or stenocardia*):ti,ab,kw

\#8 MeSH descriptor: [Myocardial Revascularization] explode all trees

\#9 ((Myocard* or cardi* or coronary) and (Revascular* or angioplast*)):ti,ab,kw

\#10 ((coronary or rotational) near atherectom*):ti,ab,kw

\#11 ("coronary artery bypass" or CABG or "aortocoronary bypass" or "coronary bypass"):ti,ab,kw

\#12 MeSH descriptor: [Percutaneous Coronary Intervention] explode all trees

\#13 ("percutaneous coronary intervention*" or PCI):ti,ab,kw

\#14 (percutaneous next coronary near/2 (interven* or revascular*)):ti,ab,kw

\#15 (stent* and (heart or cardiac*)):ti,ab,kw

\#16 coronary near (disease* or bypass or thrombo* or angioplast*):ti,ab,kw

\#17 (PTCA or "percutaneous transluminal coronary angioplasty"):ti,ab,kw

\#18 MeSH descriptor: [Heart Bypass, Right] explode all trees

\#19 ("heart manual"):ti,ab,kw

\#20 $\quad$ or \#4-\#19\}

\#21 \#3 and \#20

Flow Chart of Study Selection Process

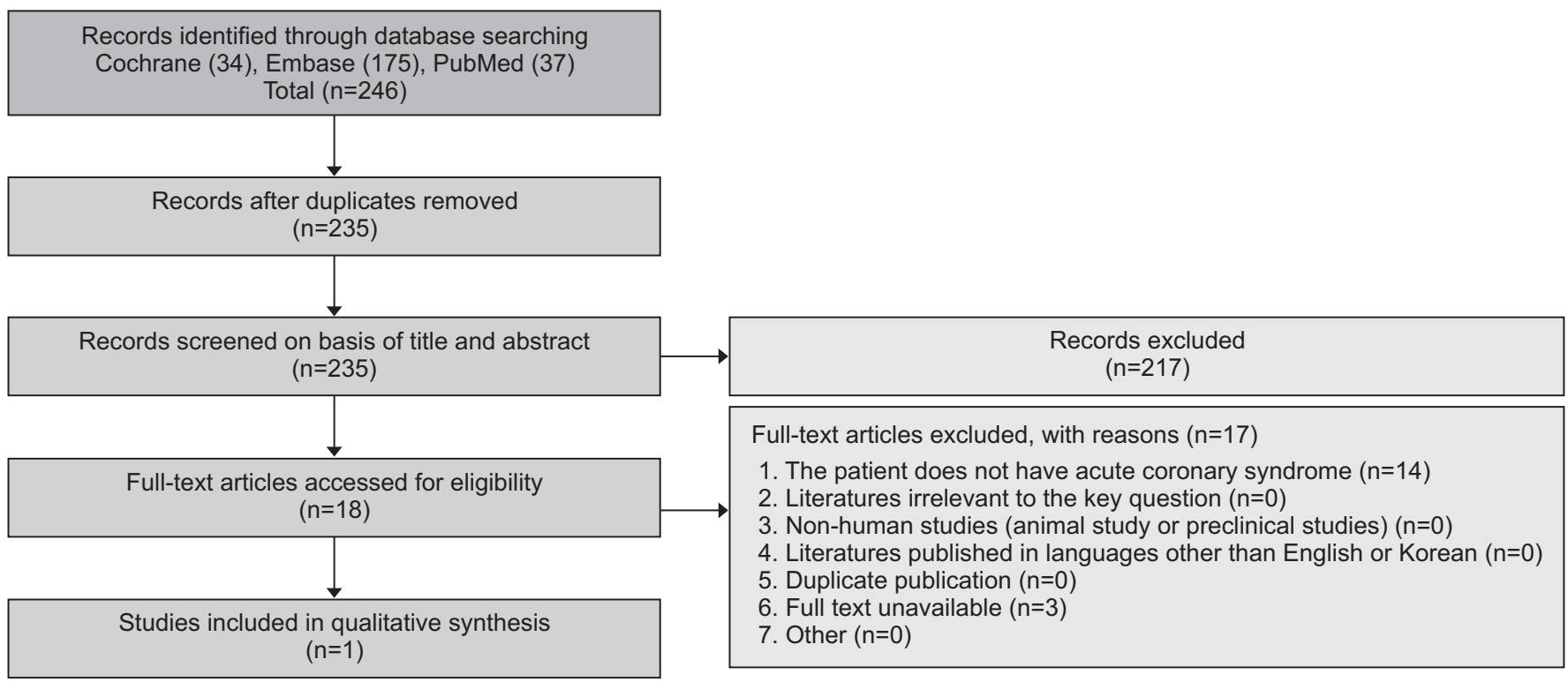

Finally Included Studies

\begin{tabular}{cc}
$\begin{array}{c}\text { Reference } \\
\text { number }\end{array}$ & \multicolumn{1}{c}{ Article } \\
\hline SR1 & $\begin{array}{l}\text { Abdelhamid A, Martin N, Bridges C, Song F, Deane KH, Hooper L. Polyunsaturated fat intake for } \\
\text { prevention of cardiovascular disease. Cochrane Database Syst Rev 2016 Sep } 7 \text { [Epub]. https://doi. } \\
\text { org//10.1002/14651858.CD012345. }\end{array}$ \\
\hline
\end{tabular}

SR, systematic reviews. 
Evidence Table for Assessment of Risk of Bias and Quality: KQ24-1

Methodological Quality of Systematic Reviews using GRADE and Level of Evidence (LOE) using SIGN Methods

\begin{tabular}{ccccccccccc}
\hline $\begin{array}{c}\text { Reference } \\
\text { number }\end{array}$ & $\mathbf{1}$ & $\mathbf{2}$ & $\mathbf{3}$ & $\mathbf{4}$ & $\mathbf{5}$ & $\mathbf{6}$ & $\mathbf{7}$ & $\mathbf{8}$ & GRADE & LOE \\
\hline SR1 & & & & & High $(4)$ & $1++$ \\
\hline
\end{tabular}

1. Limitation, 2. Inconsistency, 3. Indirectness, 4. Imprecision, 5. Publication Bias, 6. Large magnitude effect, 7. All plausible confounding would reduce a demonstrated effect or suggest a spurious effect when results show no effect, 8 . Dose response gradient.

For KQ24-2, policosanol: Only policosanol was used for the search due to a lack of relevant literature.

\section{Searching Strategies}

\#1 (policosanol or polycosanol).ti,ab

Flow Chart of Study Selection Process

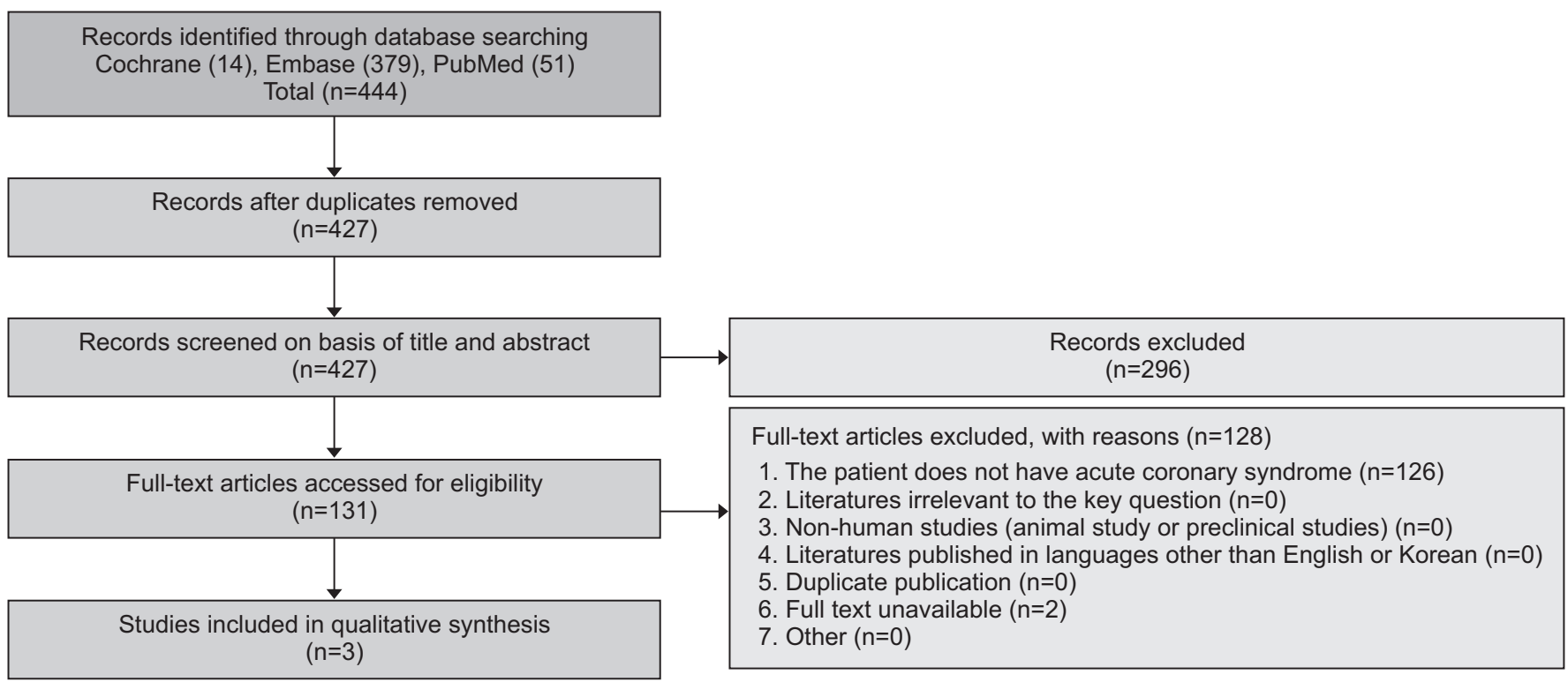


Finally Included Studies

\begin{tabular}{cc}
$\begin{array}{c}\text { Reference } \\
\text { number }\end{array}$ & Article \\
\hline RCT1 & $\begin{array}{c}\text { Marazzi G, Campolongo G, Pelliccia F, Quattrino S, Vitale C, Cacciotti L, et al. Comparison of low-dose } \\
\text { statin versus low-dose statin + Armolipid plus in high-intensity statin-intolerant patients with a pre- } \\
\text { vious coronary event and percutaneous coronary intervention (ADHERENCE Trial). Am J Cardiol }\end{array}$ \\
& $\begin{array}{c}\text { 2017;120:893-7. } \\
\text { RCT2 }\end{array}$ \\
$\begin{array}{c}\text { Marazzi G, Pelliccia F, Campolongo G, Quattrino S, Cacciotti L, Volterrani M, et al. Usefulness of nutra- } \\
\text { ceuticals (Armolipid Plus) versus ezetimibe and combination in statin-intolerant patients with dyslipid- } \\
\text { emia with coronary heart disease. Am J Cardiol 2015;116:1798-801. }\end{array}$ \\
RCT3 $\begin{array}{c}\text { Xu K, Liu X, Li Y, Wang Y, Zang H, Guo L, et al. Safety and efficacy of policosanol in patients with high on- } \\
\text { treatment platelet reactivity after drug-eluting stent implantation: two-year follow-up results. Cardio- } \\
\text { vasc Ther 2016;34:337-42. }\end{array}$ \\
\hline
\end{tabular}

RCT, randomized controlled trial.

Evidence Table for Assessment of Risk of Bias and Quality

Randomized Control Trials (RCTs)

\begin{tabular}{ccccccccc}
\hline $\begin{array}{c}\text { Reference } \\
\text { number }\end{array}$ & $\mathbf{1}$ & $\mathbf{2}$ & $\mathbf{3}$ & $\mathbf{4}$ & $\mathbf{5}$ & $\mathbf{6}$ & $\mathbf{7}$ & \multirow{2}{*}{ LOE } \\
\hline RCT1 & L & U & H & L & L & L & L & $1+$ \\
RCT2 & L & U & H & L & L & L & L & $1+$ \\
RCT3 & U & U & U & U & L & U & L & $1-$ \\
\hline
\end{tabular}

1. Random sequence generation: selection bias (biased allocation to interventions) due to inadequate generation of a randomized. 2. Allocation concealment: selection bias (biased allocation to interventions) due to inadequate concealment of allocations prior to assignment. 3. Blinding of participants and personnel: performance bias due to knowledge of the allocated interventions by participants and personnel During the study. 4. Blinding of outcome assessment: detection bias due to knowledge of the allocated interventions by outcome assessors. 5. Incomplete outcome data: attrition bias due to amount, nature or handling of incomplete outcome data. 6. Selective reporting: reporting bias due to selective outcome reporting. 7. Other bias: bias due to problems not covered elsewhere in the table.

$\mathrm{L}$, low risk of bias; $\mathrm{H}$, high risk of bias; $\mathrm{U}$, unclear risk of bias.

For KQ24-3, antioxidants: The following searching strategy was used without combining the common searching strategy:

\section{Searching Strategies}

\#1 antioxidant

\#2 MeSH descriptor: [Antioxidants] explode all trees

\#3 $\quad$ or \#1-\#2\}

\#4 MeSH descriptor: [Myocardial Ischemia] explode all trees

\#5 ((myocard* or heart* or coronary or cardia*) and (infarct* or isch* or attack*)):ti,ab,kw

\#6 ("acute coronary syndrome" or ACS):ti,ab,kw

\#7 (angina or stenocardia*):ti,ab,kw

\#8 MeSH descriptor: [Myocardial Revascularization] explode all trees

\#9 ((Myocard* or cardi* or coronary) and (Revascular* or angioplast*)):ti,ab,kw

\#10 ((coronary or rotational) near atherectom*):ti,ab,kw

\#11 ("coronary artery bypass" or CABG or "aortocoronary bypass" or "coronary bypass"):ti,ab,kw 
\#12 MeSH descriptor: [Percutaneous Coronary Intervention] explode all trees

\#13 ("percutaneous coronary intervention*" or PCI):ti, ab,kw

\#14 (percutaneous next coronary near/2 (interven* or revascular*)):ti,ab,kw

\#15 (stent* and (heart or cardiac*)):ti,ab,kw

\#16 coronary near (disease* or bypass or thrombo* or angioplast*):ti,ab,kw

\#17 (PTCA or "percutaneous transluminal coronary angioplasty"):ti,ab,kw

\#18 MeSH descriptor: [Heart Bypass, Right] explode all trees

\#19 ("heart manual"):ti,ab,kw

\#20 \{or \#4-\#19\}

\#21 \#3 and \#20

Flow Chart of Study Selection Process

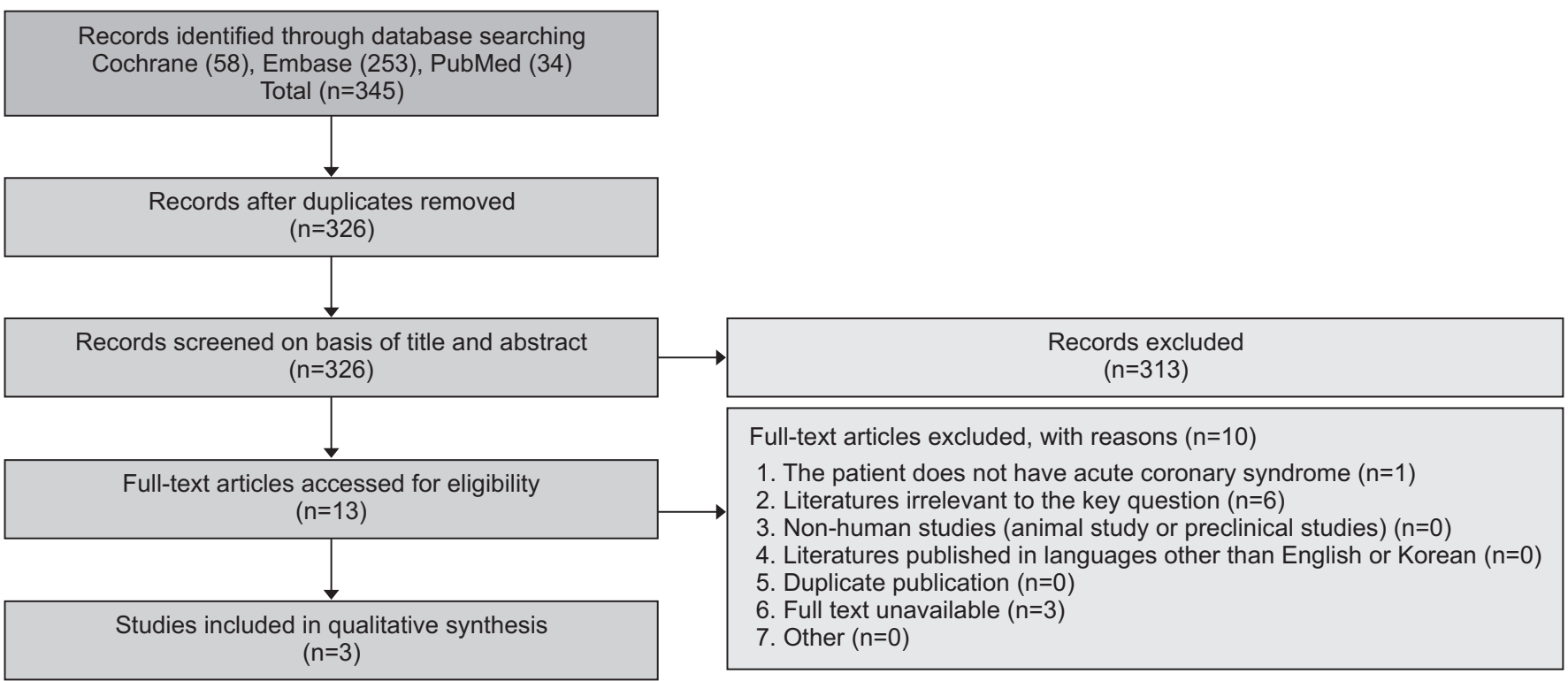

Finally Included Studies

\begin{tabular}{cc}
\hline $\begin{array}{c}\text { Reference } \\
\text { number }\end{array}$ & Article \\
\hline SR1 & $\begin{array}{c}\text { Loffredo L, Perri L, Di Castelnuovo A, Iacoviello L, De Gaetano G, Violi F. Supplementation with vitamin } \\
\text { E alone is associated with reduced myocardial infarction: a meta-analysis. Nutr Metab Cardiovasc Dis } \\
2015 ; 25: 354-63 .\end{array}$ \\
& $\begin{array}{c}\text { Myung SK, Ju W, Cho B, Oh SW, Park SM, Koo BK, et al. Efficacy of vitamin and antioxidant supplements } \\
\text { in prevention of cardiovascular disease: systematic review and meta-analysis of randomized controlled } \\
\text { trials. BMJ 2013;346:f10. }\end{array}$ \\
& $\begin{array}{c}\text { Ye Y, Li J, Yuan Z. Effect of antioxidant vitamin supplementation on cardiovascular outcomes: a meta- } \\
\text { analysis of randomized controlled trials. PLoS One 2013;8:e56803. }\end{array}$ \\
\hline
\end{tabular}

SR, systematic reviews. 
Chul Kim, et al.

Evidence Table for Assessment of Risk of Bias and Quality

Methodological Quality of Systematic Reviews using GRADE and Level of Evidence (LOE) using SIGN Methods

\begin{tabular}{|c|c|c|c|c|c|c|c|c|c|c|}
\hline $\begin{array}{l}\text { Reference } \\
\text { Number }\end{array}$ & 1 & 2 & 3 & 4 & 5 & 6 & 7 & 8 & GRADE & LOE \\
\hline SR1 & & & 1 & 1 & & & & & Low (2) & $1-$ \\
\hline SR2 & & & 1 & & & & & & Moderate (3) & $1+$ \\
\hline SR3 & & & & & & & & & High (4) & $1++$ \\
\hline
\end{tabular}

1. Limitation, 2. Inconsistency, 3. Indirectness, 4. Imprecision, 5. Publication Bias, 6. Large magnitude effect, 7. All plausible confounding would reduce a demonstrated effect or suggest a spurious effect when results show no effect, 8 . Dose response gradient.

The following is the final Cochrane Library searching strategy used for KQ25 ("Would ICT-based modality be helpful in maintaining the effects of education in the long-term?") including the basic searching strategy for education for cardiac rehabilitation:

\section{Searching Strategies}

\#1 MeSH descriptor: [Social Media] explode all trees

\#2 (social next medi*):ti,ab,kw

\#3 (twitter or facebook):ti,ab,kw

\#4 (web next $2^{*}$ or web2*):ti,ab,kw

\#5 $\{$ or \#1-\#4\}

\#6 pedometer*:ti,ab,kw

\#7 (activity monitor* or activity track* or acceleromet* or fitness monitor* or fitness track*):ti,ab,kw

\#8 (fitbit or fitband or "fit band" or fitness next watch*):ti,ab,kw

\#9 $\quad$ or \#6-\#8\}

\#10 MeSH descriptor: [Mobile Applications] explode all trees

\#11 (mobile app* or portable electronic app* or portable software app*):ti,ab,kw

\#12 (virtual realit* or exergam* or exer gam* or wifit or wi fit):ti,ab,kw

\#13 \{or \#10-\#12\}

\#14 (interactive near/2 (technol* or software)):ti,ab,kw

\#15 \{or \#14\}

\#16 MeSH descriptor: [Telemedicine] explode all trees

\#17 (telehealth* or tele-health* or telemedicine* or tele-medicine*):ti,ab,kw

\#18 (mhealth or m-health or mobile next health*):ti,ab,kw

\#19 \{or \#16-\#18\}

\#20 \#5 or \#9 or \#13 or \#15 or \#19 
Flow Chart of Study Selection Process

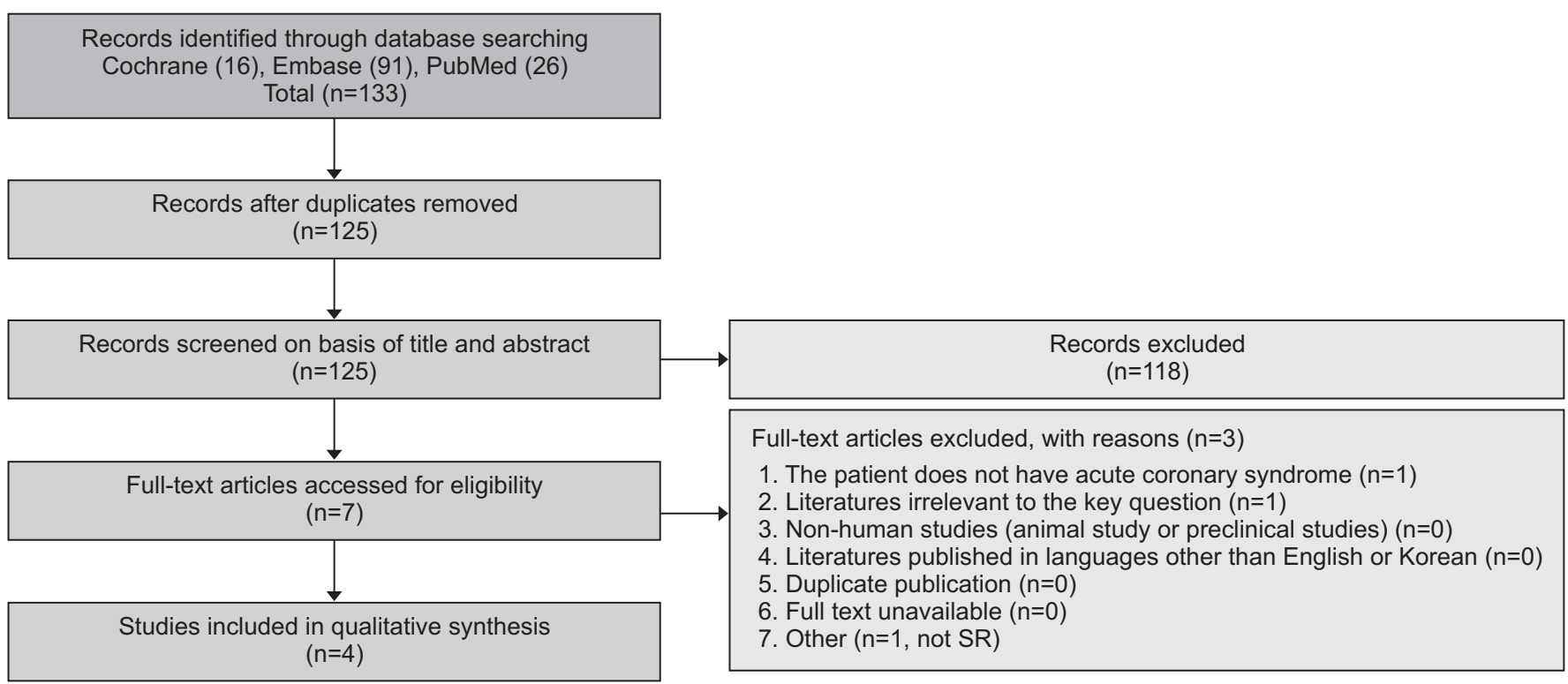

Finally Included Studies

\begin{tabular}{cc}
\hline $\begin{array}{c}\text { Reference } \\
\text { number }\end{array}$ & Article \\
\hline SR1 & $\begin{array}{c}\text { Coorey GM, Neubeck L, Mulley J, Redfern J. Effectiveness, acceptability and usefulness of mobile applica- } \\
\text { tions for cardiovascular disease self-management: systematic review with meta-synthesis of quantita- } \\
\text { tive and qualitative data. Eur J Prev Cardiol 2018;25:505-21. }\end{array}$ \\
\hline SR2 & $\begin{array}{c}\text { Gandapur Y, Kianoush S, Kelli HM, Misra S, Urrea B, Blaha MJ, et al. The role of mHealth for improving } \\
\text { medication adherence in patients with cardiovascular disease: a systematic review. Eur Heart J Qual } \\
\text { Care Clin Outcomes 2016;2:237-44. }\end{array}$ \\
SR3 & $\begin{array}{c}\text { Hamilton SJ, Mills B, Birch EM, Thompson SC. Smartphones in the secondary prevention of cardiovascu- } \\
\text { lar disease: a systematic review. BMC Cardiovasc Disord 2018;18:25. }\end{array}$ \\
SR4 & $\begin{array}{c}\text { Rawstorn JC, Gant N, Direito A, Beckmann C, Maddison R. Telehealth exercise-based cardiac rehabilita- } \\
\text { tion: a systematic review and meta-analysis. Heart 2016;102:1183-92. }\end{array}$ \\
\hline
\end{tabular}

SR, systematic reviews.

Evidence Table for Assessment of Risk of Bias and Quality

Methodological Quality of Systematic Reviews using GRADE and Level of Evidence (LOE) using SIGN Methods

\begin{tabular}{|c|c|c|c|c|c|c|c|c|c|c|}
\hline $\begin{array}{l}\text { Reference } \\
\text { number }\end{array}$ & 1 & 2 & 3 & 4 & 5 & 6 & 7 & 8 & GRADE & LOE \\
\hline SR1 & 1 & & 1 & 1 & & & & & Very low (1) & $1-$ \\
\hline SR2 & 1 & & 1 & 1 & & & & & Very low (1) & $1-$ \\
\hline SR3 & & 1 & 1 & 1 & & & & & Very low (1) & $1-$ \\
\hline SR4 & & & 1 & & & & & & Moderate (3) & $1+$ \\
\hline
\end{tabular}

1. Limitation, 2. Inconsistency, 3. Indirectness, 4. Imprecision, 5. Publication Bias, 6. Large magnitude effect, 7. All plausible confounding would reduce a demonstrated effect or suggest a spurious effect when results show no effect, 8 . Dose response gradient. 
Korean Literature Search and Selection

Korean literatures were searched on RISS, KMbase, and KoreaMed, using terms "심장재활" and "cardiac rehabilitation."

Flow Chart of Study Selection Process

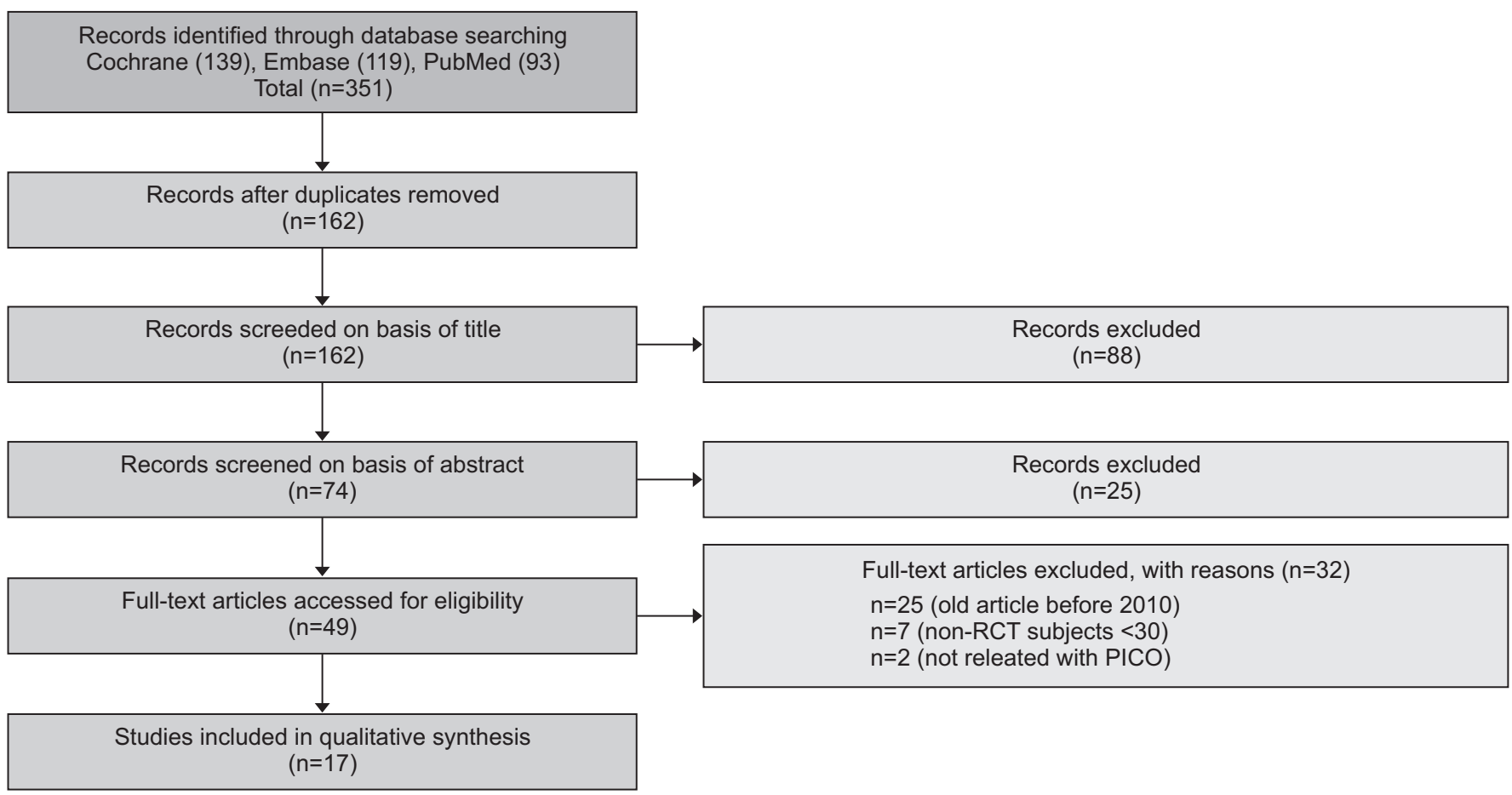

\section{Previous Published Clinical Guideline Search}

\section{PubMed Search}

1.1 Guidelines in PubMed were searched using the term "cardiac rehabilitation."

Search "cardiac rehabilitation" [TIAB] OR "cardiac rehabilitation" [MeSH] Filters: Consensus Development Conference; Guideline; NIH; Practice Guideline

PubMed date: February 2, 2018

Search results: 86

1.2 Guidelines in PubMed were searched using specific key terms such as "myocardial infarction."

Search "cardiac rehabilitation" [TIAB] OR "cardiac rehabilitation" [MeSH] Filters: Consensus Development Conference; Guideline; NIH; Practice Guideline

PubMed date: February 2, 2018

Search results: $68 \rightarrow 22$ were selected after reviewing the title and abstract

2. Review the Reference Lists of Two Recent Systemic Reviews of Cardiac Rehabilitation Guidelines 
2.1 Abel et al. article: 59 articles

Abell B, Glasziou P, Hoffmann T. Exploration of the methodological quality and clinical usefulness of a cross-sectional sample of published guidance about exercise training and physical activity for the secondary prevention of coronary heart disease. BMC Cardiovasc Disord 2017;17:153.

2.2 Seron article: 9 articles

Seron P, Lanas F, Ríos E, Bonfill X, Alonso-Coello P. Evaluation of the quality of clinical guidelines for cardiac rehabilitation: a critical review. J Cardiopulm Rehabil Prev 2015;35:1-12.

3. Search of the Database Recorded in the Guideline

Search term: "cardiac rehabilitation"

Search date: January 15, 2018

\begin{tabular}{|c|c|c|c|}
\hline No. & Guideline & Homepage URL & $\begin{array}{l}\text { Search } \\
\text { results }\end{array}$ \\
\hline 1 & $\begin{array}{l}\text { National Electronic Library for Health (NeLH) } \\
\text { Guidelines Finder }\end{array}$ & https://www.evidence.nhs.uk/ & 89 \\
\hline 2 & National Guidelines Clearinghouse & https://www.guideline.gov & 10 \\
\hline 3 & Scottish Intercollegiate Guidelines Network (SIGN) & https://www.sign.ac.uk/ & 2 \\
\hline 4 & Guidelines International Network (GIN) & http://www.g-i-n.net/ & 5 \\
\hline 5 & $\begin{array}{l}\text { Canadian Medical Association (CMA) Infobase } \\
\text { (Canadian guidelines) }\end{array}$ & $\begin{array}{l}\text { https://www.cma.ca/en/Pages/cpg-by-special- } \\
\text { ty.aspx?categoryCode=CARDIO }\end{array}$ & 1 \\
\hline 6 & $\begin{array}{l}\text { National Health and Medical Research Council } \\
\text { (NHMRC) }\end{array}$ & https://www.nhmrc.gov.au/guidelines/search & 1 \\
\hline 7 & $\begin{array}{l}\text { Clinical Practice Guidelines (Australian Guide- } \\
\text { lines) }\end{array}$ & https://www.clinicalguidelines.gov.au/ & 0 \\
\hline 8 & New Zealand Guidelines Group & $\begin{array}{l}\text { https://www.health.govt.nz/about-ministry/minis- } \\
\text { try-health-websites/new-zealand-guidelines-group }\end{array}$ & 0 \\
\hline 9 & BMJ Clinical Evidence & http://clinicalevidence.bmj.com/ & 17 \\
\hline 10 & Cochrane Database of Systematic Reviews (CDSR) & $\begin{array}{l}\text { http://onlinelibrary.wiley.com/cochraneli- } \\
\text { brary/search/ }\end{array}$ & 16 \\
\hline 11 & Database of Abstracts of Reviews of Effects (DARE) & $\begin{array}{l}\text { http://onlinelibrary.wiley.com/cochraneli- } \\
\text { brary/search/ }\end{array}$ & 25 \\
\hline 12 & Heath Technology Assessment Database (HTA) & $\begin{array}{l}\text { http://onlinelibrary.wiley.com/cochraneli- } \\
\text { brary/search/ }\end{array}$ & 19 \\
\hline
\end{tabular}

